\title{
Transverse Combustion Instabilities: Acoustic, Fluid Mechanic, and Flame Processes
}

\author{
Jacqueline O'Connor, \\ Assistant Professor, Mechanical and Nuclear Engineering, \\ Center for Combustion, Power and Propulsion, Pennsylvania State University \\ 111 Research East Building, University Park, PA 16802 \\ Phone: +1 (814) 863-1502, email: jxo22@engr.psu.edu \\ Vishal Acharya, \\ Research Engineer-II, School of Aerospace Engineering, \\ Aerospace Combustion Lab, Georgia Institute of Technology, \\ 635 Strong St NW, Atlanta, GA 30318 \\ Phone: +1 (404) 944-3647, email: vishal.sa@gatech.edu \\ Timothy Lieuwen*, \\ Professor, School of Aerospace Engineering, \\ Georgia Institute of Technology, 270 Ferst Dr, Atlanta, GA 30332 \\ Phone: +1 (404) 894-3041, email: tim.lieuwen@aerospace.gatech.edu \\ Fax: +1 (404) 385-7117
}

* Corresponding author 


\section{Abstract}

Thermoacoustic oscillations associated with transverse acoustic modes are routinely encountered in combustion chambers. While a large literature on this topic exists for rockets, no systematic reviews of transverse oscillations are available for airbreathing systems, such as in boilers, aircraft engines, jet engine augmentors, or power generating gas turbines. This paper reviews work on the problem for air-breathing systems, summarizing experimental, modeling, and active control studies of transverse oscillations. It then details the key physical processes controlling these oscillations by describing transverse acoustic wave motions, the effect of transverse acoustic waves on hydrodynamic instabilities, and the influence of acoustic and hydrodynamic fluid motions on the unsteady heat release. This paper particularly emphasizes the distinctions between the direct and indirect effect of transverse wave motions, by arguing that the dominant effect of the transverse acoustics is to act as the "clock" that controls the frequency and modal structure of the disturbance field. However, in many instances, it is the indirect axial flow disturbances at the nozzles (driven by pressure oscillations from the transverse mode), and the vortices that they excite, that cause the dominant heat release rate oscillations. Throughout the review, we discuss issues associated with simulating or scaling instabilities, either in subscale experimental geometries or by attempting to understand instability physics using identical nozzle hardware during axial oscillations of the same frequency as the transverse mode of interest. This review closes with a model problem that integrates many of these controlling elements, as well as recommendations for future research needs. Keywords: combustion instability, transverse modes, flame response 


\section{Contents}

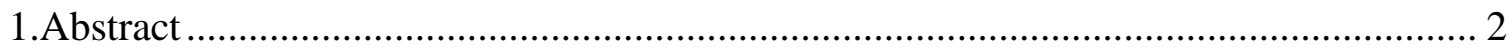

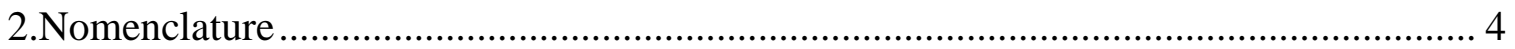

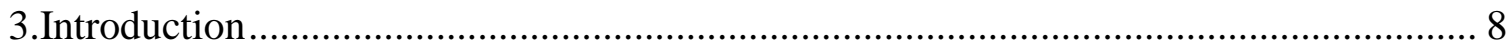

3.1. Transverse oscillations in liquid rockets ................................................. 14

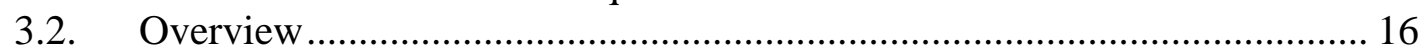

4.Experimental, Modeling, and Combustion Control Efforts......................................... 19

4.1. Experimental Investigations................................................................ 19

4.2. Modeling of transverse oscillations ………………................................ 24

4.3. Control of transverse oscillations........................................................... 30

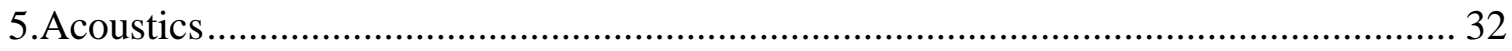

5.1. Transverse Modes in Circular Geometries ............................................... 32

5.2. Multi-Dimensional and Boundary Condition Effects ................................... 39

5.2.1. Multi-dimensional geometry effects - Injector Coupling.................. 39

5.2.2. Axial Temperature Inhomogeneity Effects ..................................... 47

5.2.3. Non-ideal Boundary Condition Effects............................................. 49

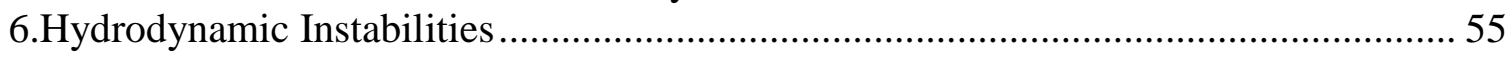

6.1. Forced Response of Hydrodynamically Unstable Flows ..............................58

6.2. Excitation Features of Transversely Forced Flows....................................... 60

6.3. Transverse forcing of typical combustor flow fields ..................................... 63

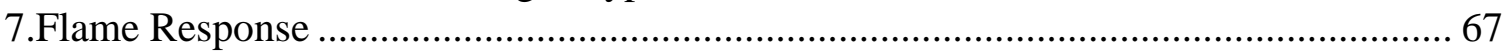

7.1. Overview of Flame Excitation Processes ..................................................... 67

7.2. Connection to Rayleigh Criterion: Role of Characteristic Times ................. 69

7.3. Response of Flames to Direct Transverse Flow Excitation $\left(\mathrm{F}_{\mathrm{T}}\right.$ pathway) ... 70

7.4. Response of Flames to Axial Flow Excitation ( $F_{L}$ pathway)........................ 71

7.5. Response of Flames to Vortical Disturbances $\left(\mathrm{F}_{\mathrm{T} \omega}\right.$ and $\left.\mathrm{F}_{\mathrm{L} \omega}\right)$..................... 72

8.System Thermoacoustic Instability Model Problem...................................................... 75

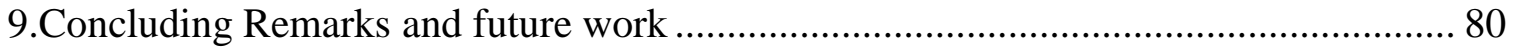

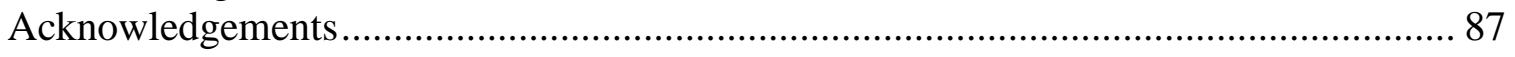

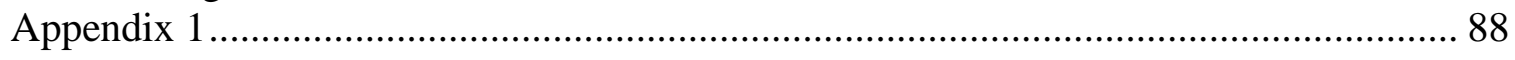

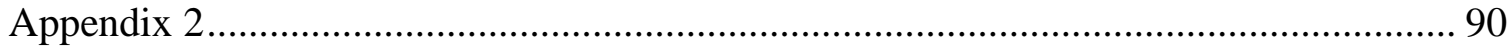

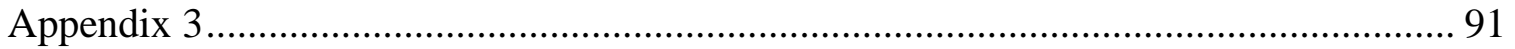

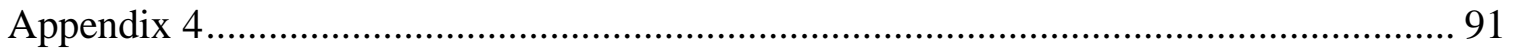

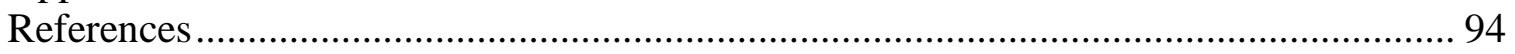

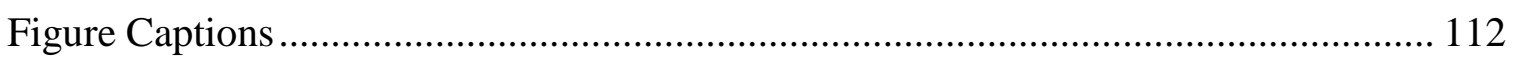

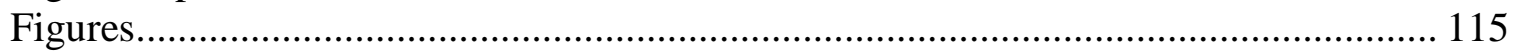

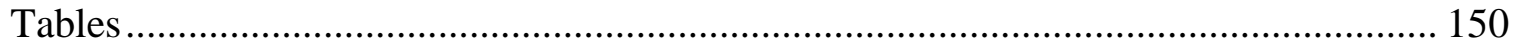




\section{Nomenclature}

$A_{c s} \quad-\quad$ Cross-sectional area

$\hat{B}_{i, m_{h}} \quad-\quad$ Complex amplitude of helical mode $m_{h}$ for velocity along coordinate $i$

$\mathscr{A}, \mathscr{B}^{-} \quad$ Acoustic wave amplitude

D - $\quad$ Diameter of inlet nozzle

Q) - Molecular diffusivity

F $\quad$ - $\quad$ Flame transfer function (FTF), defined in Eq.(6)

G - $\quad$ Level-set iso-contour variable

He - Helmholtz number, $=\omega R / c_{0}$

$L \quad$ - $\quad$ Axial length of model annular combustor

$L_{\theta} \quad-\quad$ Circumference of annular combustor based on average radius, $R$.

$L_{i} \quad-\quad$ Axial location of interface of temperature jump

$L_{f} \quad-\quad$ Flame height

$M \quad-\quad$ Mach number, $=u_{0} / c_{0}$

$\dot{Q} \quad$ - $\quad$ Global, spatially integrated, unsteady heat release rate

$R \quad$ - Average radius of annular combustor, $=a_{1}+a_{2} / 2$

$S \quad-\quad$ Swirl number

St $\quad$ - $\quad$ Strouhal number, $=\omega L_{f} / u_{0}$

$T \quad-\quad$ Temperature

$T_{a} \quad-\quad$ Acoustic time period 
$V \quad$ - $\quad$ Combustor control volume

$\hat{Z}_{o}, \hat{Z}_{\text {out }}{ }^{-} \quad$ Acoustic impedance at burner inflow and combustor exit, respectively.

$\hat{Z}_{t r} \quad-\quad$ Translated impedance

z - $\quad$ Non-dimensional mixture fraction for non-premixed flames.

$Z_{s t} \quad-\quad$ Stoichiometric mixture fraction, $=1 / 1+\phi_{O x}$

$a_{1}, a_{2} \quad$ Inner and outer radius of annular combustor respectively

$c \quad-\quad$ Speed of sound

$d_{s} \quad$ - Nozzle/burner/injector spacing

$f \quad-\quad$ Frequency

$h \quad$ - $\quad$ Axial length of inlet nozzle section

$h_{R} \quad-\quad$ Heat of reaction

$j, n \quad$ - Radial and axial acoustic mode number, respectively.

$k, k_{h} \quad-\quad$ Spatial wavenumber for acoustics and hydrodynamics, respectively.

$m_{a}, m_{h}{ }^{-} \quad$ Azimuthal mode number for acoustics and hydrodynamics, respectively.

$\dot{m}_{F}^{\prime \prime} \quad$ - $\quad$ Mass burning rate per unit area of flame surface

$n \quad-\quad$ FTF gain for thermo-acoustics model in Sec. 8.

$p \quad-\quad$ Pressure

$\dot{q} \quad$ - $\quad$ Unsteady heat release rate per unit volume

$r \quad-\quad$ Radial coordinate

$u_{i} \quad$ - $\quad$ Velocity along coordinate direction $i$

$s_{L} \quad-\quad$ Laminar flame speed 

$s \quad-\quad$ Nozzle index in annular combustor, in Figure 2 and Sec. 8
$t \quad-\quad$ Time
$x, y \quad-\quad$ Cartesian coordinates for transverse direction
$z \quad-\quad$ Axial coordinate

Greek Letters

$\Phi \quad$ - $\quad$ Phasing between unsteady heat release and unsteady pressure

$\alpha \quad$ - $\quad$ Aspect ratio of annular combustor, $=L / R$

$\beta \quad-\quad$ Ratio of $L_{i} / L$

$\kappa \quad-\quad$ Temperature ratio between burnt and unburnt gases

$\phi \quad-\quad$ Equivalence ratio

$\phi_{O x} \quad-\quad$ Stoichiometric mass ratio of oxidizer to fuel.

$\varphi \quad-\quad$ Local azimuthal angle for cylindrical coordinate centered on a nozzle

$\lambda \quad-\quad$ Acoustic wavelength

$\mu \quad-\quad$ Temporal damping coefficient in $\exp -\mu t$

$\vartheta \quad$ - $\quad$ Time-varying phase for acoustic wave

$\rho \quad-\quad$ Density

$\theta \quad-\quad$ Global azimuthal coordinate for annular combustor

$\tau \quad-\quad$ Time-delay parameter

$\omega \quad-\quad$ Angular frequency, $=2 \pi f$

$\chi \quad-\quad$ Flame aspect ratio, $2 L_{f} / D$ 


\section{Subcripts and Superscripts}

o - Stagnation value

u - Quantity in unburnt region

$b \quad$ - $\quad$ Quantity in burnt region

$0 \quad$ - $\quad$ Time-averaged component

1 - Unsteady first order perturbation component

- $\quad$ Fourier transformed variable

- $\quad$ Non-dimensionalized variable

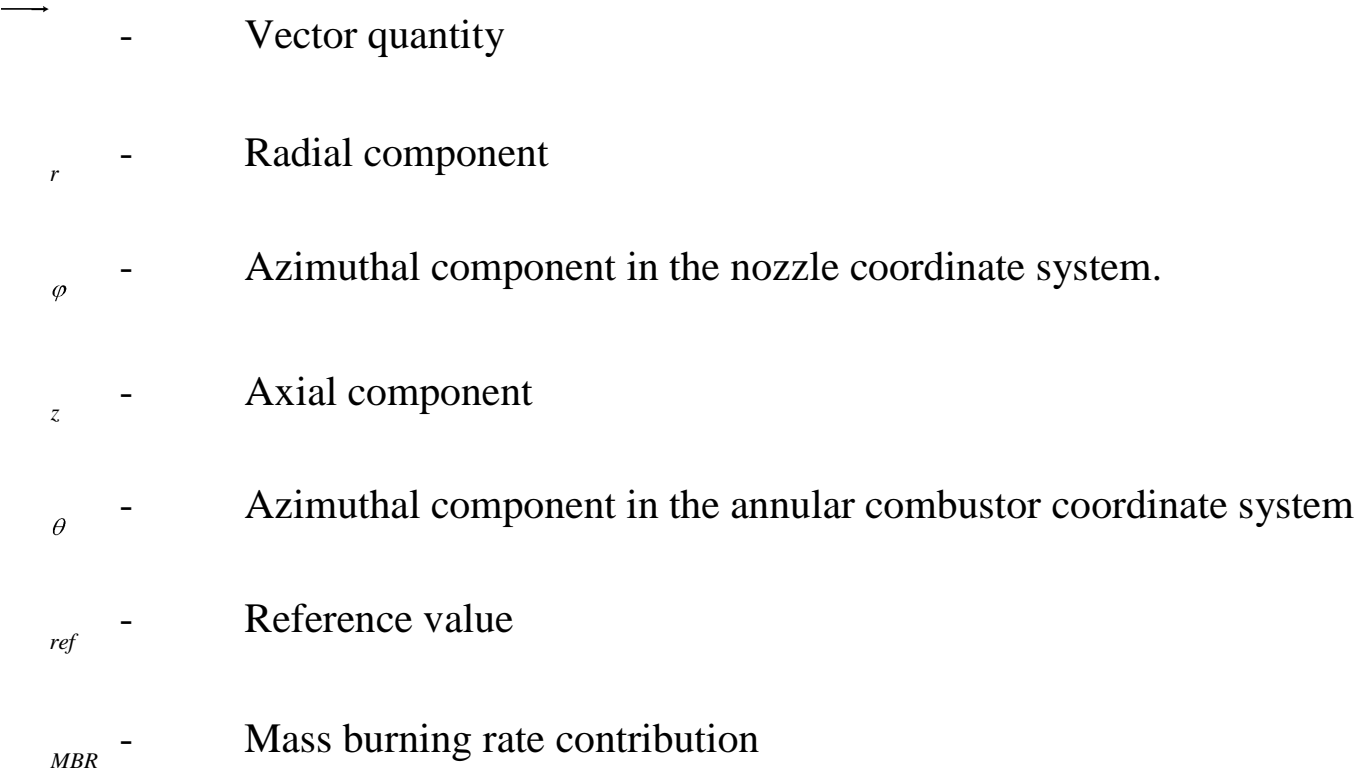




\section{Introduction}

This paper reviews the current state of understanding of transverse combustion instabilities, which are problematic in a variety of systems. For example, approaches for meeting increasingly stringent emissions regulations and efficiency demands have led to thermoacoustic oscillation problems in gas turbines used for power generation and propulsion. In particular, combustors running in a lean, premixed mode are highly susceptible to self-excited, combustion driven oscillations. This is in part due to the loss in acoustic damping associated with the secondary air inlet holes that are present in conventional diffusion flame combustors. For example, transverse oscillations in annular aircraft or aeroderivative combustors have been described by General Electric, Pratt \& Whitney, Solar Turbines, and Rolls Royce [1-4]. Similarly, transverse acoustic oscillations in annular frame-engine combustors have been described by Siemens and Alstom [5, 6]. Transverse acoustic oscillations in can-type combustors are generally higher frequency than in annular systems (the exception being radial modes in annular combustors) and have been described by Sewell and Sobieski [7]. Finally, transverse oscillations have long been an issue in jet engine augmentors [8-13]. Referred to as "screech" because of its high-pitched tone, the transverse mode in augmentors can be excited in addition to longitudinal acoustic oscillations, referred to as "rumble" or "buzz" [14]. Transverse oscillations have also been problematic in numerous rocket systems. While we note analogies and commonalities in physics throughout, the focus of this review is on air-breathing combustors and does not attempt to comprehensively address the large literature on transverse oscillations in rockets. 
Combustion instability, a coupling between resonant combustor acoustics and heat release rate fluctuations, is one of the leading challenges in developing and operating both aircraft and power-generation gas turbines [1]. Combustion instability is manifested by acoustic and heat release rate oscillations inside the combustor chamber. This feedback cycle between combustor acoustics and heat release rate oscillations typically involves the three steps outlined in Figure 1. Heat release rate fluctuations add energy to the acoustic field, leading to acoustic pressure and velocity fluctuations that propagate throughout the combustor. These acoustic fluctuations then excite vortical structures and fuel/air ratio oscillations that, in turn, lead to further heat release fluctuations that close the feedback loop.

A necessary condition for the oscillations to be self-excited is that net energy must be added to the acoustic disturbance field by the heat release. It can be shown that this occurs when the phase of the heat release rate and pressure oscillations is within \pm 90 degrees, i.e., the "Rayleigh criterion" [15]. This criterion is stated in a more general time domain formulation in Eq.(1), which indicates that the product of the heat release rate oscillation and the pressure oscillation integrated over an acoustic cycle must be greater than zero for the heat release to add energy to the acoustic field ${ }^{1}$.

$$
\iint_{V} p_{1} \vec{x}, t \quad \dot{q}_{1} \vec{x}, t d t d V>0
$$

Physically, the criterion states that the oscillating heat release, which results in oscillations in local gas dilatation, transfers energy to the acoustic field by periodically doing work on the gas when the oscillations in heat release and pressure are in phase.

\footnotetext{
${ }^{1}$ Note that unsteady heat release also adds energy to entropy disturbances. In addition, other acoustic source terms also arise in the disturbance energy equation, but are generally much smaller in magnitude for high Reynolds number flows [16-18].
} 
The conditions under which a system is linearly unstable, and the resultant amplitude of the acoustic oscillations, are dependent on the balance between disturbance growth and damping in the combustor system. In an undamped system, the value of the integral in Eq.(1) must exceed zero for oscillations to grow. However, all real systems possess some acoustic damping, and so the value of the integral must be greater than the volume-integrated system damping. Combustor damping sources include losses at inflow and outflow boundaries, and flow oscillations through cooling holes in the combustor liner excite vortices, transferring acoustic energy to vortical flow motion [19]. Similarly, narrowband acoustic damping at known problematic frequencies is often added with quarter-wave tubes and Helmholtz resonators. These damping methods are discussed further in Sec. 4.3.

When small amplitude oscillations are self-excited in linearly unstable systems, the amplitude initially grows exponentially in time. However, nonlinear effects modify the energy balance at higher amplitudes, causing the amplitude to saturate into a limit cycle, or some more complex orbit in the phase plane. For example, the unsteady heat release oscillations often do not grow linearly with acoustic disturbance amplitude, such as induced by saturation in flame area fluctuations [20].

Even if the oscillations are not self-excited (i.e., if the heat release rate oscillations do not add energy to the acoustic field, or if the rate of energy addition does not exceed system damping), significant oscillations can occur at resonant frequencies in damped systems. These noise-driven oscillations can be understood by considering the excitation of a slightly damped resonator with broadband noise, which can achieve very large amplitudes for small damping rates $[17,21]$. 
Combustion driven oscillations can be associated with any of the natural acoustic modes of the combustor system, including the longitudinal or the transverse modes - both radial and azimuthal. By focusing on transverse acoustic disturbances, this review concentrates on the physics associated with acoustic oscillations perpendicular to the mean-flow direction in the combustor. This is in contrast to longitudinal oscillations, where acoustic fluctuations oscillate in the direction of mean flow. Longitudinal oscillations have been the focus of a number of studies; see reviews by Lieuwen and Yang [1], McManus et al. [22], Ducruix et al. [23], Huang and Yang [24], and Candel et al. [25]. While the dominant coupling mechanisms between the acoustics and the flame response, reviewed later in this section, are nominally the same between longitudinal and transverse oscillations, the symmetry of the acoustic disturbance is significantly different in these two cases. Longitudinal modes excite symmetric disturbances along the flame, meaning that the acoustic disturbance amplitude is roughly constant at all azimuthal locations around the flame. However, this is not true during transverse oscillations, where the incident acoustic oscillations are non-axisymmetric with respect to the downstream axis of the flame. This introduces new coupling pathways and additional considerations. Since combustion driven oscillations are associated with an unstable combustor $\underline{\text { system}}$, another important distinction between longitudinal and transverse oscillations is the ability to accurately simulate them in sub-scale (laboratory) environments. Such approaches are routinely used for longitudinal mode studies, such as by studying the dynamics of a single or small number of nozzles, and can be done with reasonable replication of the acoustic mode structure. However, such sub-scale arrangements are generally more challenging for transverse oscillations. 
The mechanisms by which acoustic fluctuations in the combustor excite flame heat release rate fluctuations have been reviewed by several authors [17, 23, 26, 27]. In general, coupling between the acoustic field and the flame takes place through one or more pathways where disturbances in the flow field, including fluctuations in velocity, pressure, or mixture composition, drive fluctuations in the flame heat release rate. We discuss these coupling pathways with regards to transverse instabilities in Section 7, but provide a basic overview here. "Velocity-coupled response" refers to the sensitivity of the heat release rate to velocity disturbances. The velocity disturbances are associated with both acoustic and vortical velocity fluctuations [28-35], where the vortical velocity fluctuations are excited by acoustic fluctuations, as discussed in Section 6. Velocity fluctuations drive flame heat release rate fluctuations through multiple pathways, including flame area fluctuations, mass burning rate fluctuations (induced by oscillatory flame stretch and/or scalar dissipation rate), and oscillatory atomization/breakup processes. Typical characteristic delay times between the excitation of the flow disturbance and the heat release are convective in nature, usually involving the time required for a vortex to convect from its point of initiation to the midpoint of the flame (see also Sec. 4.2 and Sec. 7)

Fluctuations in mixture composition, or equivalence ratio, are common in airbreathing systems as the fuel and air flow rates are typically sensitive to acoustic disturbances. Thus, acoustic fluctuations in the combustion chamber drive fluctuations in fuel flow rate into the combustor, resulting in oscillations in local equivalence ratio [1, 36-41]. These variations in equivalence ratio drive heat release rate fluctuations through a number of pathways, including oscillations in the mixture heat of reaction and burning 
rate. Typical characteristic delay times between the excitation of the fuel/air disturbance and the heat release are also convective in nature, associated with the convection time from the fuel injection point to the midpoint of the flame (e.g., see Eq.(7)).

In addition, acoustic oscillations drive heat release rate oscillations through fluctuations in pressure, temperature, and density, which are isentropically related in the acoustic field. In air-breathing systems, the effect of pressure fluctuations is often of $O(M)$ relative to velocity and fuel/air ratio coupling and, thus, is weak in low Mach number flows [17, 42-47]. In liquid-fueled systems, fluctuations in pressure or velocity may also drive variation in fuel injection, atomization, and vaporization rates, further leading to fluctuations in local equivalence ratio; much of the literature on this liquiddriven coupling mechanism focuses on liquid-rocket applications, although a smaller general literature focuses on the response of sprays and droplets [48-50].

Users and operators of air-breathing systems often categorize combustion instabilities by their frequency. For example, "high frequency" instabilities are often termed "screech," and "low frequency" ones are referred to as "rumble" or "growl". While this distinction is useful in identifying different ranges in practice, it does not necessarily imply that key coupling physics are different. A more physics-based distinction is based upon relative time and length scales; in particular, the flame response time scale relative time to the acoustic period (the flame Strouhal number, $S t$, in Eq.(35)) and the acoustic wavelength relative to the nozzle dimension or flame length - a flame that is short relative to a wavelength is referred to as "acoustically compact". For acoustically compact flames, the distribution of the unsteady heat release is usually not important; rather, the spatially integrated unsteady heat release controls stability limits. 
In contrast, the spatial distribution of the unsteady heat release is very important in noncompact flames, as it is the relative phase of the local heat release and pressure that controls the value of the Rayleigh integral in Eq.(1).

\subsection{Transverse oscillations in liquid rockets}

Transverse oscillations have been the focus of significant investigation in liquid rockets. They are addressed in a number of books and monographs, including Yang and Anderson [51], Crocco et al. [52], Harrje and Reardon [53], and Dranovsky [54]. Initially, large-scale rocket testing revealed several issues, including mid- to highfrequency oscillations driven by a coupling between acoustic disturbances and flow and/or combustion processes. These oscillations were often abated with the use of baffles, Helmholtz resonators, and alterations to spray and impingement patterns. Initially, design of these abatement systems was largely empirical, but better understanding of the structure of the acoustic mode, particularly the transverse acoustic modes, reduced the number of design iterations required. Although the baffle and resonator solutions proved useful, questions regarding the underlying coupling mechanisms were often unanswered.

Several laboratory experiments have been designed to replicate the dominant transverse mode measured in the rocket combustion chambers, enabling a more detailed understanding of instability mechanisms [51, 55-67]. Test configurations include both single- and multiple-injector configurations in a variety of high-pressure chambers. A number of the laboratory combustors are rectangular; in this configuration, the injectors are located on one side of the chamber and the port for acoustic forcing is on a 
perpendicular face. Some chambers, however, are cylindrical, including those for both high- and low-pressure testing. The flame is typically forced with a siren [64]. This configuration allows for a high back-pressure on the forcing apparatus in order to achieve significant excitation amplitudes, as well as frequency selection by variation of the siren's rotation speed.

In addition, Reardon and coworkers $[68,69]$ developed a "sector motor" in which only a fixed sector angle of the engine was tested by using copper inserts that blocked portions of the chamber. For example, a combustor was fabricated that consisted of a $180^{\circ}$ chamber (half the chamber), that displayed only the standing form of an azimuthal mode. They also tested angles of 20, 60, 90, 120, 210, and 270 degrees, and found that changing the angle of their variable angle sector motor caused transition from stable operation at small angles, to a self-excited " $1 T$ " mode, then to a self-excited " $2 T$ " mode for angles larger than $180^{\circ}$.

These facilities enabled insight into physical mechanisms responsible for selfexcited oscillations. "Injector coupling," meaning the acoustic coupling between the pressure oscillations at the injector face associated with the acoustic mode in the chamber and oscillations in reactant mass flow rates from the injector elements, was identified as a significant mechanism [70]. For example, low pressure-drop injectors were shown to be highly susceptible to thermoacoustic oscillations; increases in pressure drop, decreasing the acoustic sensitivity of the nozzle flow rate, reduced the oscillation magnitude [71].

However, other mechanisms are also important. For example, one set of tests showed that increasing the pressure drop did not eliminate the oscillations. Rather, in that case, disturbance amplitudes decreased by increasing the velocity ratio between the 
fuel and oxidizer streams in a co-axial injector configuration [71]. This, along with positive results stemming from recessing one stream, increasing the hydrogen temperature, and increasing the thickness of the oxidizer tube, all suggest the additional role of coupling of acoustic fluctuations and jet hydrodynamic instabilities. Finally, jet breakup, atomization, and subsequent mixing of liquid fuel and oxidizer streams also profoundly influence thermoacoustic instability limits by affecting time delays between injection and ignition [72-74].

While much research in the area of combustion instability in rockets is on-going, many lessons from this research can be applied to air-breathing architectures. For example, an analogue of injector coupling is similarly a dominant mechanism of transverse oscillations in annular combustors [75, 76], and is the focus of Sec. 5.2.1. Additionally, variations in liquid jet breakup, atomization, and mixing are similarly important coupling mechanism in liquid-fueled combustors [77].

\subsection{Overview}

As described above, there is a large literature on transverse oscillations in rocket environments. However, we are not aware of a focused review of the problem in airbreathing systems. The objective of this review is to summarize, synthesize, and suggest areas for needed work for air-breathing systems, particularly gas turbines. While this review addresses the general issues associated with instabilities in both can and annular systems, it has a disproportionally larger treatment of annular combustors, where azimuthal disturbances are often the dominant mode of combustion instability. The simple reason for this focus is that annular systems are the subject of the majority of the open literature on the topic. 
Figure 2 shows a conceptual model of an annular combustor, outlining the major geometric, acoustic, and flame features. We start with the common upstream compressor discharge plenum (not shown), which discharges into a ring of fuel nozzles. Here, only a single ring of nozzles is shown, although multiple rings do exist in fielded configurations [78]. Nozzle ' $s$ ' has a cross sectional area, $A_{c s, s}$, a length $h$, and a nozzle spacing $d_{s}$. While the plenum has its own acoustic characteristics that may also support azimuthal disturbances (e.g., see Bauerheim et al. [79]), for simplicity we parameterize everything upstream of the nozzle by the impedance boundary condition, $\hat{Z}_{o}$, which is a function of operating condition, frequency, and acoustics of the upstream plenum. The flow-field created by these nozzles is often highly complex and hydrodynamically unstable, and the acoustic flow oscillations generally excite large scale vortical disturbances that also enter the problem. The flames, shown in blue, are located downstream of the nozzle outlet.

The bolded black line in the figure indicates a transverse distance. An important simplification that we will utilize in several places is that the cross sectional area of each inlet nozzle is much smaller than that of an axially oriented cut through the combustor, i.e., $A_{c s, s} / A_{c s, A} \square 1$. In this case, the unsteady volume flow rate associated with transverse flow oscillations is generally much larger than the volume flow oscillations in the nozzles, enabling us to decouple their acoustics in many instances. In this way, the azimuthal acoustic fluctuations act as a "clock," setting the overall frequency of the disturbance. These disturbances excite axial motions in the nozzle, which are often the dominant source of flame response. 
This acoustic boundary condition at the exit of the combustor is denoted as $\hat{Z}_{\text {out }}$. Parameterizing the outflow by this local boundary condition is an approximation that is accurate in cases where the nozzle dimensions are small relative to the acoustic wavelength [80].

Although no corresponding figure is included, many similar considerations apply for a can-combustion system. A key difference however, is that the cross sectional area of the nozzles is on the same order of magnitude as that of the combustor can, so that the area ratio argument described above does not apply. In this case, the acoustics of the region up and downstream of the flame are integrally linked into an overall system mode, with a mode structure that cannot be decoupled. This renders the can-combustor problem significantly more complicated.

The rest of this paper is organized as follows. Section 4 provides a review of the experimental, analytical, and modeling studies of transverse combustion instabilities in air-breathing combustor systems. In addition to experimental and computational studies, we discuss investigations into the control of transverse combustion instabilities. Then, subsequent sections describe the key physical processes during transverse combustion instabilities, with a discussion of acoustic field structure of transverse oscillations (Sec. 5), excitation of hydrodynamic instabilities by transverse forcing (Sec. 6), flame response to transverse modes (Sec. 7), and an illustrative model problem for transverse thermoacoustic instability (Sec. 8). We conclude by identifying key gaps in understanding, and recommendations for future research topics. 


\section{Experimental, Modeling, and Combustion Control Efforts}

This section summarizes experimental, modeling, and instability control efforts in the area of transverse combustion instability for gas turbine applications, which are listed in Figure Captions 
Figure 1 - Thermoacoustic feedback cycle.

Figure 2 - Conceptual model of an annular combustor undergoing azimuthal combustion instability.

Figure 3 - Model annular combustor configurations from (a) Worth and Dawson [102] and (b) Bourgouin et al. [104].

Figure 4 - Conceptual drawing of transverse velocity coupled combustion instability (a), and spark-schlieren photograph of the resulting oscillations (b), reproduced from Rogers and Marble [9].

Figure 5 - Quartz cylindrical combustor at atmospheric conditions for high-frequency, self-excited oscillation studies from Schwing et al. [84].

Figure 6 - Conceptual view of the General Electric tunable combustor acoustic (TCA) test rig with a perforated piston to vary plenum length (A), single injector (B), combustion chamber with flame (C), and perforated piston to vary combustor length (D), adapted from Mongia et al. [78].

Figure 7 - Combustor design concept for transversely forced experiments, Adapted from Malanoski et al. [91].

Figure 8 - Transverse forcing facilities in the unwrapped annular combustor configuration with high aspect ratio combustors and speaker tubes: (a) Experiment of O'Connor and Lieuwen [87], and (b) Experiment of Malanoski et al. [92].

Figure 9 - (a) Acoustic forcing configuration and (b) acoustic field measurements for the transverse forcing facility of Lespinasse $e t$ al. [98]. Filled symbols are acoustic pressure measurements and open symbols are acoustic velocity measurements.

Figure 10 - T-shape transverse forcing experiments from (a) Hauser et al. [95] and (b) Saurabh and Paschereit [99], capable of both transverse and longitudinal forcing.

Figure 11 - Extent of flame interaction in an annular combustor measured via timeaverage $\mathrm{OH}^{*}$ chemiluminescence from an overhead view. Images show two nozzle spacings - (a) 2.33 and (b) 1.56 times the nozzle diameter - where all swirlers have a counterclockwise rotation direction. Reproduced from Worth and Dawson [102].

Figure 12 - Pressure (left) and temperature (right) fields from LES simulation of a fullannular combustor during azimuthal acoustic mode oscillations. Reproduced from Wolf et al. [134].

Figure 13 - General Electric LM6000 DLE combustor cross-section with damper tube in combustor plenum and circumferential baffles. Reproduced from Mongia et al. [78]. 
Figure 14 - Dependence of (a) azimuthal and (b) radial natural frequencies of annular geometry upon normalized gap width; following Lieuwen [195].

Figure 15 - Joint probability of forward and backward spinning wave amplitudes for an $m_{a}=1$ azimuthal instability in a model annular combustor with a) counterclockwise swirling nozzles and b) alternating swirl between clockwise and counterclockwise, and c) two baffles placed at $180^{\circ}$ angles around the annulus. Adapted from Worth and Dawson $[102,103]$.

Figure 16 - Joint probability density functions of standing wave amplitudes in an azimuthally unstable annular combustor at three noise levels. Adapted from Noiray et al. [120].

Figure 17 - Standing acoustic pressure and velocity fields shown relative to inflow nozzle. The $x$-coordinate corresponds to the azimuthal coordinate $\theta$ in the full annular combustor, where $x=R \theta$.

Figure 18 - Schematic of transverse pressure fluctuations as experienced by nozzle for pressure anti-node (a) and pressure node (b) forcing cases.

Figure 19 - Pressure amplitudes at the center plane $(y=0)$ for $D / \lambda \approx 0.04$ (400 Hz) with (a) an anechoic nozzle for the pressure node case, and (b) pressure release condition at the nozzle for the pressure anti-node case. Reproduced from Blimbaum et al. [195].

Figure 20 - Experimental results of PIV measurements of velocity (vectors) and normalized vorticity (color) fluctuation for $400 \mathrm{~Hz}$ asymmetric (a) and $400 \mathrm{~Hz}$ symmetric (b) forcing in non-reacting swirling flow at $u_{z, 0}=10 \mathrm{~m} / \mathrm{s}, S=0.85$. Experimental flame luminescence images of $400 \mathrm{~Hz}$ asymmetric (c) and $400 \mathrm{~Hz}$ symmetric (d) forcing of a flame in a swirling flow at $u_{z, 0}=10 \mathrm{~m} / \mathrm{s}, S=0.5$, and an equivalence ratio of 0.9 . Arrows point to the wrinkles resulting from c) helical and d) ring vortices.

Figure 21 - Examples of symmetric and asymmetric flame oscillations in an annular combustor. Flames displaying (a) symmetric (ring vortex) and (b) asymmetric (helical vortex) response are shown using the fluctuation amplitude of $\mathrm{OH}^{*}$ chemiluminescence imaging from an overhead view [103]. In (c) and (d), OH-PLIF images of flames at pressure anti-nodes and velocity anti-nodes, respectively, with arrows showing similar flame wrinkling to Figure 20 [162].

Figure 22 - Simplified representation of Figure 2 for a model thin annular combustor with a temperature discontinuity interface.

Figure 23 - Effect of azimuthal modes $(m)$ on the normalized natural frequency in a thin annular duct with $L / R=0.5$ and $\kappa=T_{b, 0} / T_{u, 0}$."PR" denotes a pressure release outflow boundary condition. 
Figure 24 - Representation of modes for $m_{h}=0$ (ring vortices), $m_{h}>0$ (clockwisewinding helix), and $m_{h}<0$ (counter-clockwise-winding helix).

Figure 25 - Linear spatial growth rate of hydrodynamic modes in a swirling jet as a function of excitation frequency. Plot courtesy of Benjamin Emerson.

Figure 26 - Top-view of nozzle showing Cartesian and cylindrical polar coordinate systems with respect to the center of the nozzle.

Figure 27 - Amplitude of helical modes excited by (a) symmetric forcing and (b) asymmetric acoustic forcing. Illustrative results from transversely forced cold-flow experiment by O'Connor and Lieuwen [87] showing modal amplitudes for $1200 \mathrm{~Hz}$ (c) symmetric forcing and (d) asymmetric forcing.

Figure 28 - Response of a circular jet to (a) symmetric and (b) asymmetric (forcing of the $m_{h}= \pm 1$ modes) acoustic forcing around the circumference of the jet. Images reproduced from Kusek et al. [250].

Figure 29. Response of a liquid coaxial jet in a transverse acoustic at a range of phases between the speakers at either end of a transverse forcing facility [271].

Figure 30 - Flame coupling pathways in transversely excited flames.

Figure 31 - Time-delays between the unsteady pressure and intermediate processes affecting the heat release: (a) Effect of transverse velocity oscillations, (b) Effect of fuel/air ratio oscillations from induced longitudinal velocity oscillations, (c) Effect of flow instabilities.

Figure 32 - Velocity fluctuations during (a) longitudinal forcing; (b) transverse forcing.

Figure 33 - Flame transfer function $(F)$ amplitude comparison between symmetric (black) and asymmetric (gray) forcing. Data reproduced from Acharya et al. [128].

Figure 34 - Cut-section side view along the circumferential direction, showing the control volume and the coordinate system.

Figure 35 - Variation of instability growth rate for the 1 case for the fundamental frequency $m_{a}=1$ and the second mode $m_{a}=2$, with the time-delay parameter $\tilde{\tau}_{q}$ for $n=1.5$. 


\section{Figures}

\subsection{Experimental Investigations}

Experimental investigations have considered self-excited and open-loop transverse forcing flame response. Flame dynamics during self-excited oscillations have been measured in both laboratory-scale annular combustors and single-nozzle configurations. Open-loop forcing studies have used single- or multi-nozzle configurations that mimic a sector of an annular combustor.

Self-excited azimuthal modes in an annular geometry were studied by Moeck and Paschereit $[106,107]$ in an annular geometry that used an array of Rijke tubes with heated wire-mesh elements at atmospheric pressure. Although no flame is present in this facility, this arrangement enables a means for manipulating heat release profiles/ symmetry and characterizing their influence on azimuthal mode behavior.

Worth and Dawson [101, 102] presented results from the annular combustor shown in Figure 3(a). This facility allows for variation in the number of nozzles (and hence nozzle spacing), direction of swirl of each nozzle, inner and outer lengths of the combustor walls, fuel type, equivalence ratio, and baffles. Both standing and spinning modes could be excited by, for example, altering the relative swirl directions in adjacent nozzles or insertion of baffles. The spin direction of the mode was driven by the alignment of the local heat release and the mean swirl direction in the annulus. 
A similar but larger annular combustor was developed by Bourgouin et al. [104], shown in Figure 3(b), and tested for both swirl-stabilized and matrix burner flames [105]. Visualization of the flame behavior showed that flame response is largely driven by transverse to longitudinal coupling at each nozzle, again showing that the transverse mode acts as a "clock" to drive the flame response. Studies from Sattelmayer and coworkers [155-158] used an annular combustor to better understand the differences in flame response to longitudinal acoustic modes in an annular and a single-flame configuration, but no direct measurements of transverse modes in this combustor appear to be published. These three annular facilities were run at atmospheric pressure.

Self-excited oscillations have also been measured in sector configurations where one or several flames in a geometry mimicking a portion of a full combustor are investigated. Several classic studies focused on transverse oscillations in augmentor configurations. Experiments by Rogers and Marble [9], Elias [10], and Kaskan and Noreen [11], all showed self-excited, transverse oscillations in single-flame, bluff-body stabilized configurations at near-atmospheric conditions. Elias also measured similar oscillations for two- and three-flame configurations. In all these studies, asymmetric vortex shedding from alternating edges of the flameholder were observed, driven by the asymmetry of the transverse acoustic mode relative to the combustor centerline. These data show synchronization between transverse velocity fluctuations in the chamber and vortex rollup on the flameholder, as can be seen in Figure 4.

High-frequency, self-excited transverse oscillations have been investigated in single-nozzle, cylindrical configurations [82-84, 159]. Sattelmayer and coworkers [8284] obtained results from a configuration shown in Figure 5 where self-excited, 
transverse ocillations were present. Like the results from Rogers and Marble [9], vortex shedding in this configuration was driven by transverse motion in the combustion chamber.

Additionally, a subset of these single-flame test sections used facilities that deliberately interrogated system behavior using longitudinal excitation at the same transverse oscillation frequencies encountered in full-scale hardware. As noted in the introduction, one of the key complexities associated with transverse oscillations is accurately simulating them in sub-scale environments. Thus, in approaches that reproduce acoustic frequencies, but not spatial acoustic mode shapes, it is worthwhile to consider what physics is and is not correctly emulated. This method of testing nozzles has been used for rocket injectors [160], and has been applied to air-breathing hardware as well. Facilities at both Solar Turbines [4] and General Electric [2] used this methodology for nozzle design screening. Solar Turbines developed the single-nozzle rig to match as many parameters as possible, including the length of the combustor, diameter and annulus height, flow velocities and inlet temperatures. A translating plug assembly downstream of the combustion zone was used to adjust the combustor length in order to match the oscillation frequency to that of the engine [161]. Similarly, General Electric used a tunable length single-nozzle rig, shown in Figure 6, to sweep through oscillation amplitude dependence as a function of frequency/chamber length and, thereby to screen nozzles at known frequencies of concern for the engine [78].

Both studies admit the short-comings of these experiments, particularly in that the flame is subjected to axial acoustic motions in the screening test rig, but transverse acoustic motions in the engine. However, at lower frequencies (100's of Hertz), the 
flame and nozzle are acoustically compact with respect to the wavelength of the transverse mode. We hypothesize that the apparent usefulness of results from this type of experiment stems from two points: (1) the much larger sensitivity of compact flames to axial flow disturbances than transverse disturbances (see Sec. 7), and (2) the dependence of the axial flow oscillations from the nozzle upon the oscillatory pressure from the transverse wave (see Sec. 5.2.1). For this reason, we hypothesize that it is the scalar pressure oscillations at the nozzle that must be replicated, something that can be done with appropriate care in an acoustically compact case by matching acoustic frequencies and nozzle impedances in either a transverse or longitudinally self-excited system.

In addition to flame response in self-excited systems, flame response to open-loop transverse acoustic forcing has been measured in a number of facilities. Typically, these combustors have been designed to mimic a thin-gapped annular combustor, as if an annular combustor had been cut at an azimuthal position and "unwrapped" to produce a high aspect ratio chamber, as shown in the diagram in Figure 7 [91]. Of course, curvature effects do render differences between this system and real annular systems, particularly in cases where the annular gap is not small relative to the overall outer radius of the annulus.

Several of these configurations have been built and operated. The first combustor, from O'Connor et al. [86], consists of a high-aspect ratio combustor with three adjustable speaker tubes on each side for acoustic forcing. The relative phasing between these drivers can be adjusted in order to simulate different standing wave disturbance fields at the nozzle. Two different combustors with this design are pictured in Figure 8. Data from this experiment has provided information about the velocity 
disturbance field present in transversely-forced, swirl-stabilized flames [76, 85, 87-89]. In particular, the authors found that the structure of the acoustic field at the nozzle (e.g., a pressure node vs. antinode), strongly the affects local and global flame response. Similar facilities have subsequently been built based on this design [91, 92, 94], all of which operate at atmospheric pressure.

Experiments by Lespinasse and coworkers $[97,98]$ used a similar high-aspectratio chamber and a single acoustic driver on either side of the axisymmetric bluff-body stabilized flame at atmospheric conditions, as shown in Figure 9(a). The distance between the walls of the acoustic forcing chamber is variable so that the chamber can be tuned to the wavelength of the forcing frequency. This is similar to the adjustable tubes in the experiments from O'Connor et al. [86], Malanoski et al. [91], and Emerson et al. [94]. The experiment enables a transverse standing wave, as is shown in the acoustic measurements in Figure 9(b). Additionally, the nozzle location within the experiment is variable so that flame response to different locations in a standing acoustic wave can be measured. Measurements showed significant differences in local and global flame response at various locations in the acoustic waveform.

Another design has been used by Hauser et al. $[95,96]$ and Saurabh and Paschereit [99], which enables both transverse and longitudinal excitation. These Tshape combustors, shown in Figure 10, have been used to study both transverse forcing and combinations of transverse and longitudinal forcing at atmospheric conditions. The combination of transverse and longitudinal excitation, or "mixed-mode" excitation, results in different flame response characteristics over a range of frequencies. Additionally, traveling wave excitation in the facility from Saurabh and Paschereit [100] 
has shown that the flame response to traveling waves is similar to that of flames at pressure anti-nodes, showing significant global heat release rate fluctuation, a topic we will address further in Sec.5.2.1.

Comparisons between sector rigs and full annular model combustors have shown that key flame dynamics during self-excited transverse oscillations can be faithfully captured in transverse forcing facilities. A comparison study by O'Connor et al. [162] showed that the symmetric and asymmetric response of the flames at the pressure antinode and node locations in an annular combustor is consistent with the symmetric and asymmetric velocity and vorticity response of the single transverse forced flame. This study explained that a transverse-forcing facility should capture four major characteristics of the annular rig in order to capture the proper flow and flame dynamics: the flow field, particularly the vortex breakdown structure; the flame shape, particularly the stabilization method; the acoustic compactness and resonances of the system; and the flame spacing if multiple flames are present in very close proximity.

Flame-flame interaction is important in closely spaced flames [101], and is illustrated in Figure 11, which compares two nozzle configurations with different nozzle spacings. At the largest spacing (Figure 11(a)), the flames retain distinct shapes, but at the smallest spacing (Figure 11(b)), they are significantly merged in the regions between the nozzles. This level of interaction can distinctly influence the flame dynamics, as will be discussed in Sec. 7.

\subsection{Modeling of transverse oscillations}

Many modeling studies of transverse oscillations have been performed for selfexcited and forced systems. Approaches include acoustic solvers, flamelet dynamics 
models, unsteady Reynolds-averaged Navier Stokes (URANS) simulations, and large eddy simulations (LES); these are summarized in Figure Captions 
Figure 1 - Thermoacoustic feedback cycle.

Figure 2 - Conceptual model of an annular combustor undergoing azimuthal combustion instability.

Figure 3 - Model annular combustor configurations from (a) Worth and Dawson [102] and (b) Bourgouin et al. [104].

Figure 4 - Conceptual drawing of transverse velocity coupled combustion instability (a), and spark-schlieren photograph of the resulting oscillations (b), reproduced from Rogers and Marble [9].

Figure 5 - Quartz cylindrical combustor at atmospheric conditions for high-frequency, self-excited oscillation studies from Schwing et al. [84].

Figure 6 - Conceptual view of the General Electric tunable combustor acoustic (TCA) test rig with a perforated piston to vary plenum length (A), single injector (B), combustion chamber with flame (C), and perforated piston to vary combustor length (D), adapted from Mongia et al. [78].

Figure 7 - Combustor design concept for transversely forced experiments, Adapted from Malanoski et al. [91].

Figure 8 - Transverse forcing facilities in the unwrapped annular combustor configuration with high aspect ratio combustors and speaker tubes: (a) Experiment of O'Connor and Lieuwen [87], and (b) Experiment of Malanoski et al. [92].

Figure 9 - (a) Acoustic forcing configuration and (b) acoustic field measurements for the transverse forcing facility of Lespinasse $e t$ al. [98]. Filled symbols are acoustic pressure measurements and open symbols are acoustic velocity measurements.

Figure 10 - T-shape transverse forcing experiments from (a) Hauser et al. [95] and (b) Saurabh and Paschereit [99], capable of both transverse and longitudinal forcing.

Figure 11 - Extent of flame interaction in an annular combustor measured via timeaverage $\mathrm{OH}^{*}$ chemiluminescence from an overhead view. Images show two nozzle spacings - (a) 2.33 and (b) 1.56 times the nozzle diameter - where all swirlers have a counterclockwise rotation direction. Reproduced from Worth and Dawson [102].

Figure 12 - Pressure (left) and temperature (right) fields from LES simulation of a fullannular combustor during azimuthal acoustic mode oscillations. Reproduced from Wolf et al. [134].

Figure 13 - General Electric LM6000 DLE combustor cross-section with damper tube in combustor plenum and circumferential baffles. Reproduced from Mongia et al. [78]. 
Figure 14 - Dependence of (a) azimuthal and (b) radial natural frequencies of annular geometry upon normalized gap width; following Lieuwen [195].

Figure 15 - Joint probability of forward and backward spinning wave amplitudes for an $m_{a}=1$ azimuthal instability in a model annular combustor with a) counterclockwise swirling nozzles and b) alternating swirl between clockwise and counterclockwise, and c) two baffles placed at $180^{\circ}$ angles around the annulus. Adapted from Worth and Dawson $[102,103]$.

Figure 16 - Joint probability density functions of standing wave amplitudes in an azimuthally unstable annular combustor at three noise levels. Adapted from Noiray et al. [120].

Figure 17 - Standing acoustic pressure and velocity fields shown relative to inflow nozzle. The $x$-coordinate corresponds to the azimuthal coordinate $\theta$ in the full annular combustor, where $x=R \theta$.

Figure 18 - Schematic of transverse pressure fluctuations as experienced by nozzle for pressure anti-node (a) and pressure node (b) forcing cases.

Figure 19 - Pressure amplitudes at the center plane $(y=0)$ for $D / \lambda \approx 0.04$ (400 Hz) with (a) an anechoic nozzle for the pressure node case, and (b) pressure release condition at the nozzle for the pressure anti-node case. Reproduced from Blimbaum et al. [195].

Figure 20 - Experimental results of PIV measurements of velocity (vectors) and normalized vorticity (color) fluctuation for $400 \mathrm{~Hz}$ asymmetric (a) and $400 \mathrm{~Hz}$ symmetric (b) forcing in non-reacting swirling flow at $u_{z, 0}=10 \mathrm{~m} / \mathrm{s}, S=0.85$. Experimental flame luminescence images of $400 \mathrm{~Hz}$ asymmetric (c) and $400 \mathrm{~Hz}$ symmetric (d) forcing of a flame in a swirling flow at $u_{z, 0}=10 \mathrm{~m} / \mathrm{s}, S=0.5$, and an equivalence ratio of 0.9 . Arrows point to the wrinkles resulting from c) helical and d) ring vortices.

Figure 21 - Examples of symmetric and asymmetric flame oscillations in an annular combustor. Flames displaying (a) symmetric (ring vortex) and (b) asymmetric (helical vortex) response are shown using the fluctuation amplitude of $\mathrm{OH}^{*}$ chemiluminescence imaging from an overhead view [103]. In (c) and (d), OH-PLIF images of flames at pressure anti-nodes and velocity anti-nodes, respectively, with arrows showing similar flame wrinkling to Figure 20 [162].

Figure 22 - Simplified representation of Figure 2 for a model thin annular combustor with a temperature discontinuity interface.

Figure 23 - Effect of azimuthal modes $(m)$ on the normalized natural frequency in a thin annular duct with $L / R=0.5$ and $\kappa=T_{b, 0} / T_{u, 0}$."PR" denotes a pressure release outflow boundary condition. 
Figure 24 - Representation of modes for $m_{h}=0$ (ring vortices), $m_{h}>0$ (clockwisewinding helix), and $m_{h}<0$ (counter-clockwise-winding helix).

Figure 25 - Linear spatial growth rate of hydrodynamic modes in a swirling jet as a function of excitation frequency. Plot courtesy of Benjamin Emerson.

Figure 26 - Top-view of nozzle showing Cartesian and cylindrical polar coordinate systems with respect to the center of the nozzle.

Figure 27 - Amplitude of helical modes excited by (a) symmetric forcing and (b) asymmetric acoustic forcing. Illustrative results from transversely forced cold-flow experiment by O'Connor and Lieuwen [87] showing modal amplitudes for $1200 \mathrm{~Hz}$ (c) symmetric forcing and (d) asymmetric forcing.

Figure 28 - Response of a circular jet to (a) symmetric and (b) asymmetric (forcing of the $m_{h}= \pm 1$ modes) acoustic forcing around the circumference of the jet. Images reproduced from Kusek et al. [250].

Figure 29. Response of a liquid coaxial jet in a transverse acoustic at a range of phases between the speakers at either end of a transverse forcing facility [271].

Figure 30 - Flame coupling pathways in transversely excited flames.

Figure 31 - Time-delays between the unsteady pressure and intermediate processes affecting the heat release: (a) Effect of transverse velocity oscillations, (b) Effect of fuel/air ratio oscillations from induced longitudinal velocity oscillations, (c) Effect of flow instabilities.

Figure 32 - Velocity fluctuations during (a) longitudinal forcing; (b) transverse forcing.

Figure 33 - Flame transfer function $(F)$ amplitude comparison between symmetric (black) and asymmetric (gray) forcing. Data reproduced from Acharya et al. [128].

Figure 34 - Cut-section side view along the circumferential direction, showing the control volume and the coordinate system.

Figure 35 - Variation of instability growth rate for the 1 case for the fundamental frequency $m_{a}=1$ and the second mode $m_{a}=2$, with the time-delay parameter $\tilde{\tau}_{q}$ for $n=1.5$. 


\section{Figures}

. These studies can be roughly grouped into those that focus on the acoustics of the system, those that focus on the flame dynamics of transversely excited systems, and those that couple the flame dynamics with a larger system level acoustics and stability model. This section briefly surveys these approaches, as key results from many of them are further detailed in the rest of this review.

A variety of methods have been used to assess the stability of combustion systems [109-112, 114, 116, 120, 121, 123-125, 163-165]. These system-level models are particularly beneficial for determining the stability and mode shapes of combustion systems, although calculating limit cycle amplitudes requires incorporation of nonlinear effects that are automatically captured in LES calculations, but not necessarily so in reduced order models. An example of a linear system modeling approach is provided in Section 8 for a transverse instability problem that is reduced to a quasi-one dimensional formulation. More generally, multi-dimensional acoustics can be captured by coupling Helmholtz solvers to flame dynamics models as described later in this section.

Low-order flame modeling has provided detailed information about local flame dynamics at a relatively low computational cost. In particular, the use of level-set formulations for premixed flames and mixture fraction formulations for non-premixed flames enables a low-dimensional description of how flamelets respond to perturbations [166-168]. In these formulations, the flamelet surface is represented by the iso-contour of an implicit function, given by $G(\vec{x}, t)$ for premixed flames and by the mixture fraction, 
$Z(\vec{x}, t)$ for nonpremixed flames. The evolution of this contour is tracked using the $G$ equation $[169,170]$ for premixed flames:

$$
\frac{\partial G}{\partial t}+\vec{u} \cdot \vec{\nabla} G=s_{L}|\vec{\nabla} G|
$$

where the instantaneous flame position follows the $G(\vec{x}, t)=0$ isocontour. Similarly, the non-premixed flame dynamics are described by the $Z$ equation, derived below assuming equal diffusivities of all species [17]:

$$
\frac{\partial Z}{\partial t}+\vec{u} \cdot \nabla Z=\nabla \cdot Q \nabla \nabla
$$

This approach can be generalized to include differential diffusion effects by replacing it with the appropriate species equations. In the fast chemistry limit, the nonpremixed flame sheet position follows the $Z(\vec{x}, t)=Z_{s t}=1 / 1+\phi_{O x}$ contour, where $\phi_{O x}$ is the stoichiometric mass ratio of oxidizer to fuel. For example, in order to consider the velocity-coupled flame response, the space-time distribution of the vortical and acoustic velocity field can be inserted into these expressions to determine the fluctuations in flame position.

Ultimately, it is heat release rate oscillations that drive thermoacoustic oscillations. The instantaneous heat release rate is spatially distributed, and it is the value of the $p_{1} \dot{q}_{1}$ product shown in Eq.(1) that determines whether energy is locally added or removed from the acoustic field by the heat release fluctuations. However, the spatially integrated heat release rate, $\dot{Q}(t)=\int_{V} \dot{q}(\vec{x}, t) d V$, has a special significance in cases where the flame region is acoustically compact, i.e., is small relative to an acoustic wavelength, and the detailed distribution of the heat release is unimportant $[171,172]$. Within the 
flamelet formulation, the spatially integrated heat release can be written as a surface integral as:

$$
\dot{Q}=\iint_{A} \dot{m}_{F}^{\prime \prime} h_{R} d A_{f}
$$

where the mass burning rate, i.e., the mass consumption rate of reactants per unit flamelet surface area, is given by:

$$
\begin{aligned}
& \text { Premixed } \rightarrow \dot{m}_{F}^{\prime \prime}=\rho_{u} s_{L} \\
& \text { Non-premixed } \rightarrow \dot{m}_{F}^{\prime \prime}=\left.\frac{\left(1+\phi_{O x}\right)^{2}}{\phi_{O x}} \rho \mathscr{O}|\vec{\nabla} z|\right|_{Z=z_{s t}}
\end{aligned}
$$

Flame transfer functions are used extensively in the literature to describe the amplitude and phase of the flame response as a function of frequency [22, 173-176]; the flame describing function provides a nonlinear extension of this concept $[177,178]$. The flame transfer function is defined as:

$$
\hat{F}(\omega)=\frac{\hat{\dot{Q}}_{1}(\omega) / \dot{Q}_{0}}{\hat{u}_{1, r e f}(\omega) / u_{0}}
$$

Where $\hat{u}_{1, \text { ref }}$ denotes some suitable reference perturbation velocity [76]. The analysis using the $F T F$ has been used for both premixed $[76,93,127,179]$ and non-premixed flames as well $[171,180,181]$. While these transfer functions can be quite complex, a commonly used simplification for the flame response is the so-called $n-\tau$ model:

$$
\dot{Q}_{1}(t)=n \quad u_{1, r e f}\left(x_{r e f}, t-\tau\right)
$$

This time-domain equation relates the unsteady heat release rate to the flow disturbances (assumed to be the velocity in this case, but could also be a superposition of other disturbances such as pressure and fuel/air ratio as well). It can be shown that the $n-\tau$ 
model accurately describes velocity-coupled premixed flame response in the $f L_{f} / u_{0} \square 1$ limit, where $\tau$ is proportional to $L_{f} / u_{0}$ (e.g., for laminar 2-D flames, $\tau=L_{f} / 2 u_{0}$ [17]). Note also that the time delay and phase of the FTF are related by $\omega \tau=\angle \hat{F}(\omega)$. Section 7 describes further the factors influencing the flame response to transverse disturbances. Models for the heat release rate can be used in larger system acoustics/disturbance models. For example, the Helmholtz equation with a heat release source term is given by:

$$
\frac{\omega^{2}}{c_{0}^{2}} \hat{p}_{1}+\rho_{0} \nabla \cdot\left(\frac{1}{\rho_{0}} \nabla \hat{p}_{1}\right)=-\frac{\gamma-1}{c_{0}^{2}} i \omega \dot{\hat{Q}}_{1}
$$

Here, $\omega$ is the eigenfrequency and $p_{1}(\vec{x}, t)=\operatorname{Re} \hat{p}_{1}(\vec{x}, \omega) \exp (-i \omega t)$. This equation neglects mean flow effects but can describe three-dimensional oscillations in arbitrarily complex geometries. Initial studies using this approach utilized simplified geometries that were one dimensional. Some of the initial studies were carried out by Merk [182], and then for industrial systems by Putnam [183], afterburners by Bloxsidge et al. [184], and a Rijke tube by Heckl [185]. A system of components whose acoustics are approximated as one-dimensional can also be coupled into a network model to describe more complex three-dimensional geometries, such as described by Bohn and Deuker [186]. More generally, finite element methods can be used to solve the acoustic wave equation for complex three dimensional geometries $[117,119]$ to calculate the eigenfrequencies of the system and disturbance growth/decay rates; e.g., Campa et al. [118] used this approach to model the acoustics of a full annular combustor configuration that consists of a plenum, burners and the combustion chamber. 
Describing nonlinear phenomenon, such as limit cycle amplitudes requires incorporation of nonlinear effects. This can be done either with full computations, or with hybrid models. For example, as discussed further in the next section, acoustic amplitudes are often sufficiently low that acoustic/gas dynamic processes remain linear, while it is heat release or boundary damping effects that are nonlinear. In this case, timedomain solutions of the linearized wave equations can be coupled with nonlinear heat release models or nonlinear boundary conditions in order to capture nonlinear phenomenon [116, 187]. For example, Graham and Dowling [126] coupled a series of quasi-one-dimensional time domain solutions to describe acoustic motions in an annular combustor, coupled to a nonlinear heat release model. A higher fidelity approach was presented by Wolf et al. [134], using a Helmholtz solver with unsteady heat release rates provided by LES.

Some URANS work has also been reported for predicting global features of flame response to transverse instabilities [131]. Large-eddy simulation has provided a detailed view of flame dynamics in both sector simulations $[6,137,138,140]$ and full-annular simulations [75, 132-134], such as shown in Figure 12. In addition to describing bulk acoustic features, such as frequency, these computations also enable description of more complex time-dependent behavior, such as nonlinear behaviors or the intermittent switching between standing and spinning modes, features that a Helmholtz solver cannot resolve. However, even in high fidelity computations, there are difficulties in predicting instability boundaries and oscillation amplitudes due to uncertainty in boundary conditions and numerical damping in the system. These issues disrupt the balance between driving and damping that determines the amplitude, or even existence, of the 
instability, and so true predictive capability has eluded the community to date. Despite these deficiencies, physical driving mechanisms can be retrieved from these data as well as trends in instability amplitude, which are important metrics for comparison between experimental data and computational prediction. For example, these simulations suggest that the dominant coupling mechanism between the transverse acoustic mode and flame heat release rate fluctuations is the injector coupling mechanism, which causes axial flow pulsations $[75,133]$.

\subsection{Control of transverse oscillations}

Control of combustion instabilities can be achieved through both active and passive means. Passive control techniques are most typically found on hardware currently in operation, and include such techniques as resonators, pilot fuel, and fuel staging. Active control is not typically found in industrial hardware, but it is noteworthy that the only field utilization of active control on a large frame engine was applied to a transverse instability [153].

Passive control techniques, including Helmholtz resonators, quarter-wave tubes, and perforated plates, are used to damp acoustic oscillations by both resistive and reactive processes [143]. Perforated plates damp acoustic oscillations by transferring fluctuating acoustic energy into vortical motion at the edges of the plate's holes. Typical combustor liner configurations contain several perforations to allow cooling air to enter the combustor, and also act to damp acoustic oscillations.

Helmholtz resonators and quarter-wave tubes are dominantly reactive devices, although the large amplitude oscillations at the resonator outlet generally also lead to an amplitude dependent resistive acoustic damping as well. Figure 13 illustrates a 
commercial combustor with quarter wave tubes in the combustor plenum used for damping transverse oscillations [78]. Damper tubes of either type are used widely across the industry [144] and have been used on rocket engines as well [53]. Design and placement of such resonant devices is critical for their effectiveness. Stow and Dowling showed that the required number of resonators must be at least one greater than the highest azimuthal acoustic mode number in order to effectively damp azimuthal oscillations [146]. While a range of resonator locations effectively damped the oscillations, the resonator efficacy is sensitive to the resonator volume.

Also shown in Figure 13 are two circumferential baffles located above and below the middle burner, labeled as "heat shields". In addition to their other functions, these shields serve as baffles for high frequency radial modes. These baffles have an analogous function to their usage for transverse rocket instabilities described in Sec. 3.1. Radially-aligned baffles were examined by Dawson and Worth [103], who showed that the addition of one or two baffles had little influence on oscillation amplitudes, but forced the acoustic mode to a standing-wave pattern, and eliminated traveling waves and some of the more random switching in wave patterns referred to in Sec. 5.1. Three or more baffles dampened the ocillation amplitude.

Fuel staging and pilot fuel addition are also common passive instability control techniques. This is a common technique for gas turbine operators in can-combustion systems to suppress oscillations, although strategies for fuel staging must be customized for each engine [7]. Fuel staging can also be used to break the symmetry of annular combustor configurations in an attempt to damp azimuthal acoustic modes; this has been show theoretically [121, 142] and experimentally [3]. Additionally, pilot fuel may be 
introduced to reduce oscillations. This strategy was described by Solar Turbines for reduction of transverse oscillations [4], while GE discussed how a fuel circuit that was originally intended to extend lean blow-out limits has been used to reduce transverse combustion instability [145]. However, fuel staging or the addition of pilot fuel usually has adverse emissions impacts because of its change of fuel/air distribution.

Siemens also presented various fuel nozzle design modifications for alteration of instability characteristics in their annular frame engines that had transverse oscillations [148]. A cylindrical burner outlet (CBO) was used to push the flame axially downstream, changing the convective time between fuel/air mixture injection and the flame region, and moving the phase between the mixture and heat release oscillations outside the positive Rayleigh index band. Asymmetric burner outlets (ABO) were also employed to alter the axial development of large-scale vortices. Symmetry breaking with various levels of asymmetry in location of CBO's and ABOs were also utilized.

Finally, active control techniques have been tested for the control of azimuthal modes. These techniques employ external excitation, either acoustic forcing or pulsing of fuel or air delivery to the combustor, to damp the self-excited mode $[148,150-152,154$, 188]. These actuators can stabilize the system by pulsing either at the forcing frequency with a phase-shift, or away from the forcing frequency.

\section{Acoustics}

\subsection{Transverse Modes in Circular Geometries}

This section summarizes transverse acoustic mode shapes and frequencies of circular combustion chambers. We will quickly summarize classical duct acoustic results, 
and refer the readers to more detailed references for further discussion $[17,189]$.

Transverse modes in rectangular geometries are a straightforward generalization of onedimensional axial modes (axial modes are denoted with the index, $n$ ) to the transverse direction, and are not discussed further. In circular geometries, the transverse motion can be associated with radial modes (denoted by $j$ ) or azimuthal modes (denoted by $\left.m_{a}\right)^{2}$. A key distinction that azimuthal modes in circular geometries have from axial and/or transverse modes in rectangular geometries, or the radial modes in circular geometries, is the possibility of traveling waves in the azimuthal direction; in contrast, standing waves are the only natural modes in rectangular geometries ${ }^{3}$. In axisymmetric systems, the direction of rotation of these traveling waves is indeterminate and waves can exist that rotate in one or both directions. Two counter-rotating waves of equal amplitude lead to a standing wave transverse field. A key distinction between a one-dimensional traveling and standing acoustic wave is that the pressure and transverse velocity in a traveling wave are in-phase with each other, as opposed to being 90 degrees offset in a standing wave. In problems where the unsteady heat release rate is sensitive to the transverse velocity, the resulting conditions under which the pressure and heat release rate are in phase and, hence, the system is unstable, will be completely different for traveling and standing waves. In most cases, however, it appears that the heat release is more sensitive to the axial velocity (see Sec. 5.2.1), whose characteristics are controlled by the unsteady pressure and, thus, the heat release is "pressure-coupled" to the transverse wave. In this situation, standing or traveling waves result in the same pressure-heat release rate phase

\footnotetext{
${ }^{2}$ However, "azimuthal modes" have a radial amplitude variation in general, except in thin annular gaps where the radial dependence is negligible.

${ }^{3}$ Traveling waves can be set up in rectangular geometries by appropriate forcing schemes on the boundaries, such as described by Saurabh and Paschereit [100].
} 
relationship. Throughout this section, we utilize a linear acoustic framework only, as acoustic amplitudes in air-breathing systems are often sufficiently low that gas dynamic nonlinearities (e.g., shocks) are negligible. However, incorporation of gas dynamic nonlinear processes is often necessary in rocket applications [190-192].

In order to analyze basic features of the natural oscillations, consider the annular combustor in Figure 2. We assume a rigid walled duct of outer radius, $a_{2}$, so that $u_{r, 1}\left(r=a_{2}\right)=0$. For annular geometries, the same boundary condition applies at the inner radius, $u_{r, 1}\left(r=a_{1}\right)=0$ where $a_{1}<a_{2}$. Annular geometries with "thin gaps", i.e., those where $\left(a_{2}-a_{1}\right) / a_{1} \square 1$, are the simplest to understand and are considered first $[112,113$, $193,194]$. In this limit, the radial dependence of the azimuthal velocity is negligible, so the azimuthal velocity is a function of the azimuthal and axial coordinate, $u_{\theta, 1}=u_{\theta, 1}(\theta, z)$. Neglecting any axial dependencies for the time being (general expressions are presented in Appendix 1, Eq.(53)), harmonic pressure and azimuthal velocity oscillations in this thin gap limit are given by:

$$
\begin{aligned}
& p_{1}(\theta, t)=\text { Real } \exp (-i \omega t) \quad \mathscr{G}_{1} \exp \left(i m_{a} \theta\right)+\mathscr{G}_{2} \exp \left(-i m_{a} \theta\right) \\
& u_{\theta, 1}(\theta, t)=\operatorname{Real}\left\{\frac{m_{a}}{\rho \omega} \exp (-i \omega t) \mathscr{G}_{1} \exp \left(i m_{a} \theta\right)+\mathscr{G}_{2} \exp \left(-i m_{a} \theta\right)\right\}
\end{aligned}
$$

The natural frequency, $f_{m}$, of purely azimuthal modes then equals that of a onedimensional duct with periodic boundary conditions whose length equals the circumference of the gap, $L_{\theta}=2 \pi a_{1}+a_{2} / 2$, and $f_{m}=m_{a} c_{0} / L_{\theta}$, where $m_{a}$ is an integer.

Similar simplifications apply for radial modes in the thin-gap limit. Specifically, the radial velocity profile and pressure-velocity relationship for the first radial mode 
approach that of a one-dimensional duct with rigid boundaries conditions, whose rigidrigid duct length is equal to the gap width, $a_{2}-a_{1}$ and $f_{j}=j-1 c_{0} / 2\left(a_{2}-a_{1}\right)$, where $j-1$ is an integer for the radial acoustic mode number ${ }^{4}$. Figure 14 plots the exact natural frequency of a duct with no axial variations in the acoustic field, normalized by its thin gap value for the first two azimuthal modes and two radial modes, showing that these approximations are quite accurate even in situations where the annular gap is not thin at all.

We next consider circular geometries (i.e., $a_{1}=0$ ). The first four transverse mode natural frequencies are:

$$
f_{m j}=\underset{1 T}{(0.29,0.49,0.61,0.67)} \frac{c_{0}}{a_{2}}
$$

The two lowest frequency transverse modes, the " $1 T$ " and " $2 T$ " or first and second tangential modes (referred to as azimuthal modes in this paper), which correspond to the $m_{a}, j=1,1$ and $m_{a}, j=2,1$ modes, and both have a $\theta$ dependence. The third frequency is the first radial mode, " $1 R$ ", i.e. $m_{a}, j=0,2$, which has no $\theta$ dependence. In the absence of mean flow in the azimuthal direction, the natural frequency of the " $1 T$ " and " $2 T$ " "azimuthal" modes are independent of whether the azimuthal dependence is standing or, if it is traveling, on the azimuthal direction of rotation.

Operational experience has shown that azimuthal modes in air-breathing combustors have a more complex time-varying dependence than the purely harmonic

\footnotetext{
${ }^{4}$ Note that the index for azimuthal modes, $m_{a}$, starts from zero, while for radial modes, it starts from one.
} 
oscillations shown in Eq.(9). However this form is still a useful model for fitting real data, such as by generalizing it to:

$$
p_{1}(\theta, t)=\mathscr{G}_{1}(t) \sin \omega t+m_{a} \theta+\vartheta_{1}(t)+\mathscr{C}_{2}(t) \sin \omega t-m_{a} \theta+\vartheta_{2}(t)
$$

where $G_{1}(t)$ and $G_{2}(t)$ are the time varying amplitudes of waves spinning in the clockwise and counterclockwise directions, respectively, and $\vartheta_{1}(t)$ and $\vartheta_{2}(t)$ denote the associated time varying phase. The pressure could also be equivalently written as:

$$
p_{1}(\theta, t)=\mathscr{G}_{\sin }(t) \sin \omega t+\vartheta_{\sin }(t) \sin m_{a} \theta+\mathscr{G}_{\cos }(t) \sin \omega t+\vartheta_{\cos }(t) \cos m_{a} \theta(12)
$$

Where $G_{\text {sin }}(t)$ and $G_{\cos }(t)$ denote the time varying amplitude of azimuthal standing waves. Note that $C_{1}(t), G_{2}(t)$ and $C_{\sin }(t), C_{\cos }(t)$ are related using standard trigonometric relations. While these two expressions are equivalent, they emphasize the traveling (spinning) wave and standing wave character of the wave forms, respectively. Moreover, if the amplitude coefficients in either expression are not equal, then the wave form could be envisioned as a standing wave with a rotating nodal pattern. For example, rotating standing waves are observed that slowly "drift" in a somewhat stochastic manner in either the clockwise or counter-clockwise direction, such as described by Evesque et al. [112]. Whether the modes are standing or spinning in engine is important for 2 reasons. Standing waves may align their nodal patterns at fixed locations in systems with azimuthal non-uniformities, such as due to azimuthal fuel staging or due to other geometric features. In such cases, if the oscillations levels are being monitored with a single pressure sensor, the azimuthal location of the sensor is unimportant if the mode is spinning, while very important in cases where it is standing. Similarly, if resonators are to be employed for control, their 
azimuthal location is critically important in how effective they are in cases where the waves are standing.

Analysis of data from annular combustors shows standing, spinning, and rotating standing wave fields $[103,134]$. For example, experiments by Worth and Dawson showed that flame spacing, nozzle swirl direction, and the placement of baffles influenced which acoustic field structure dominated $[102,103]$. Figure 15 shows an example of these results, plotting the joint probability of the clockwise and counterclockwise spinning wave amplitudes for two different swirler configurations and one baffled configuration. The bivariate PDF (Probability Density Function) in Figure 15(a) is bimodal, indicating that the acoustic mode switches between a standing pattern and a counter-clockwise spinning wave during the experiment. In contrast, the mode is more likely to be standing when the swirl direction is alternated between adjacent nozzles, as shown in Figure 15(b). However, the bivariate PDF of this standing mode has significant spread due to the lack of azimuthal boundary condition, indicating that the acoustic mode likely "drifts" in the azimuthal direction during the experiment. When a baffle is inserted in the combustor, the baffle boundary condition prevents this drift and locks the standing mode into one location, as is shown in Figure 15(c). Here, the bivariate PDF is concentrated on the standing-wave axis.

Similarly, results from other annular combustors have shown both standing and traveling waves $[106,107,111,115,134]$ and several investigators have analyzed the reason for the preference of one modal structure over another [109, 120, 122]. Schuermans et al. [122] showed that although linear analysis of axial-velocity coupled flames showed no preference for either type of disturbance, nonlinear effects tended to 
favor traveling waves. Ghirardo and Juniper [109] showed that the sensitivity of the flame to azimuthal velocity fluctuations also plays an important role in determining the standing/spinning nature of the mode - this sensitivity can be understood from the discussion earlier in this section, where it was noted that the pressure and transverse velocity are in phase for traveling waves, and shifted ninety degrees relative to each other for standing waves. In addition, Bauerheim et al. [79, 135, 136] showed that even for flames that are only sensitive to axial disturbances, a preference for standing/spinning waves can exist in linear analyses when azimuthal mean flow and plenum acoustics are also considered.

Noiray and Schuermans [120] showed that not only is the acoustic mode dependent on the flame's sensitivity to the velocity disturbance field, but also the noise level in the combustor. Figure 16 shows a result from their analysis, similar to those from Figure 15. Here, the two amplitudes plotted on the axes are standing wave amplitudes, as opposed to traveling amplitudes in Figure 15.

However, these traveling/standing distinctions seem to become ambiguous in the highly stochastic environments found in real systems. Noiray and Schuermans [120] also noted that the broad width of the wave amplitude PDF, as shown in Figure 16, is associated with intermittent switching between traveling and standing wave motions. This can be seen by comparing the PDF of spinning wave amplitudes (Eq.(11)) and standing wave amplitudes (Eq.(12)), and noting that both seem equally reasonable representations of data at high disturbance amplitudes. They introduced a stochastic model with a tunable additive noise parameter, showing this effect. 
To summarize these studies, the relative preference for standing or traveling disturbances is influenced by the linear flame sensitivity to flow disturbances, nonlinear effects, mean azimuthal flow effects, geometry effects (such as plenum), and noise effects.

\subsection{Multi-Dimensional and Boundary Condition Effects}

The prior section summarized key results for classical duct acoustics where the wave field could be reduced to a one- or two-dimensional problem, and the axial distribution of the wave field was not considered. However, combustors intrinsically involve more complex geometries and boundary conditions, as well as non-uniform temperature fields. We consider three important problems in this section - multidimensional geometry effects, effects of an axial temperature gradient, and effects of an impedance boundary condition on azimuthal modes.

\subsubsection{Multi-dimensional geometry effects - Injector Coupling}

This section introduces multi-dimensional effects in combustors where the acoustic field is dominated by transverse modes. This discussion is particularly focused around annular geometries, although it also has relevance to can-combustors. Specifically, having discussed the basic acoustic features of annular and circular geometries, we next consider the additional effects associated with transverse wave motion interaction with the inflow nozzles, an example of which is shown in Figure 17. In order to motivate the key questions considered in this section, refer to the annular combustor or transverse forcing rig sketched in Figure 2 or Figure 7, showing an annular ring around which nozzles are placed. These nozzles could be situated at any point in the 
standing wave field. For example, Figure 18 shows a nozzle situated near the velocity node and another near the pressure node, which experience significantly different disturbance fields and flame excitation physics. Moreover, these disturbance wavelengths could be quite long relative to the nozzle dimension in the case of annular modes, or on the same order of the wavelength for radial modes.

As will be shown in Sec. 7, several paths exist by which a transverse mode may excite a flame $[86,127,196,197]$. In particular, transverse modes directly excite the flame, they excite hydrodynamic flow instabilities, and they also lead to axial acoustic flow oscillations in the nozzle. It is these axial acoustic flow oscillations that are the focus of this section. Axial acoustic oscillations are a wave diffraction effect, as the dominantly transverse mode leads to an oscillatory pressure field across the nozzle.

We begin this section by highlighting some of the purely acoustic aspects of this problem for cases where the nozzle diameter is small relative to an acoustic wavelength. We closely follow Blimbaum et al. [195], which presented three-dimensional acoustic simulations of a geometry similar to Figure 17 . This geometry consists of a high aspect ratio combustor, simulating an "unwrapped" annulus, connecting into an annular nozzle (such as in Figure 8(a)). An important limiting case is where the nozzle cross-sectional area is small relative to the radial-axial cross-sectional area cut of the annulus, $A_{c s, s} / A_{c s, A} \square 1$, as also discussed in the context of Figure 2. In this case, the azimuthal distribution of volume flow rate oscillations induced by transverse acoustic disturbances are only weakly influenced by the nozzle itself. In this limit the pressure and axial velocity at the nozzle outlet are controlled by the general waveform of the disturbance in the absence of the nozzle, i.e., the location of the nozzle with respect to global velocity or 
pressure nodes. Blimbaum et al. [195] demonstrated this by comparing three different azimuthal acoustic field scenarios: "pressure anti-node", "pressure node", and "traveling wave" scenarios. The first two disturbance fields lead to standing wave fields in the system, where the nozzle outlet centerline is nominally located at a pressure anti-node and node, respectively. The velocity field exhibits a node and antinode, respectively. In the traveling wave forcing scenario, an anechoic boundary is applied to one side of the domain. In the absence of the nozzle, the acoustic field is one-dimensional.

The pressure anti-node case generates zero transverse velocity at the nozzle outlet centerline, but large pressure fluctuations are present that are symmetric across the nozzle centerline. These pressure fluctuations lead to nearly axisymmetric, axial velocity disturbances. The nozzle response for this symmetric forcing case can be understood from quasi one-dimensional concepts. In contrast, the pressure node case exhibits large transverse velocity fluctuations in the center of the combustor. Because of the centerline pressure node, the pressure fluctuations have a 180 degree phase difference on the two sides of the nozzle centerline. This tends to drive axial velocity fluctuations that are phased approximately 180 degrees apart on the left and right sides of the nozzle (but smaller in amplitude as the pressure fluctuations are small if the nozzle is acoustically compact). This nozzle response is intrinsically three-dimensional. Both cases are illustrated in Figure 18. The traveling wave case shows an intermediate behavior (since a traveling wave is the superposition of two standing waves).

The nozzle impedance has a significant effect on this transverse to axial coupling for pressure anti-node and traveling wave acoustic excitation. In order to understand this point, assume that the nozzle impedance is given by $\hat{Z}_{o}$ at the lower end of the nozzle 
section, $z=-h$, as shown in Figure 17. This nozzle impedance, $\hat{Z}_{o}$, is associated with all of the acoustic properties of the flow passages, swirlers, and compressor discharge plenum, upstream of this point [125] (Note however, that it may not be possible to specify an impedance boundary condition of this form in cases where transverse motions are excited in the upstream plenum). As long as the frequency lies below the cutoff frequency of the inflow nozzle, the acoustic field quickly reverts to one-dimensional, as transverse disturbances are evanescent. A one-dimensional field leads to a powerful simplification, as the relationship between the axial velocity and pressure in the nozzle at all other axial locations are uniquely related through the impedance translation theorem [198]:

$$
\frac{\hat{Z}_{t r}(z, \omega)}{\rho_{0} c_{0}}=\frac{\hat{p}_{1}(z, \omega)}{\hat{u}_{z, 1}(z, \omega)}=-\left[\frac{\left(-\frac{\hat{Z}_{o}}{\rho_{0} c_{0}}-1\right) e^{-i k z+h}+\left(-\frac{\hat{Z}_{o}}{\rho_{0} c_{0}}+1\right) e^{i k z+h}}{\left(\frac{\hat{Z}_{o}}{\rho_{0} c_{0}}+1\right) e^{-i k z+h}+\left(-\frac{\hat{Z}_{o}}{\rho_{0} c_{0}}+1\right) e^{i k z+h}}\right]
$$

In other words, for transverse mode frequencies that are much lower than nozzle cutoff frequencies, it is not the nozzle impedance, $\hat{Z}_{0}$, or nozzle length, $\boldsymbol{h}$, parameters that are individually important, but their net translated impedance, $\hat{Z}_{t r}$ at the nozzle outlet. We consider three important limits for $\hat{Z}_{t r}(z=0)$ : pressure release $\left(\hat{Z}_{t r} / \rho_{0} c_{0}<<1\right)$, anechoic $\left(\hat{Z}_{t r} / \rho_{0} c_{0}=1\right)$, and rigid boundary conditions $\left(\hat{Z}_{t r} / \rho_{0} c_{0}>>1\right)$, leading to a matrix of $3 \times 3$ different scenarios which are summarized in Table 2 and discussed further next [195]. 
In this table, "pressure-coupled" means that the axial acoustic velocity and pressure at the nozzle outlet are related through the translated impedance, $\hat{Z}_{t r}$, in the $D / \lambda \square 1$ limit. In this case, the nozzle outlet axial velocity is approximately given by:

$$
\hat{u}_{z, 1}(z=0, \omega)=\frac{\hat{p}_{1}(z=0, \omega)}{\hat{Z}_{t r}(z=0, \omega)}
$$

In contrast, the "Not pressure coupled" results mean that the pressure and axial velocity are not related in this simple way. Although the axial velocity fluctuations at the nozzle outlet may be non-negligible in this out of phase case, they are of opposite phases on the opposite sides of the nozzle and cancel each other, leading to a progressive decay in disturbance amplitude and fluctuation energy in the nozzle. For this reason, the nozzle impedance has no influence on the disturbance field characteristics in the pressure node case. This result has important implications for radial modes in an annular combustor where nozzles located along the radial centerline are located at a velocity antinode/pressure node.

Returning to the table, "Quasi 1-D" means that the pressure fluctuations from the azimuthal mode at the nozzle outlet is controlled by the "bulk" acoustics of the combustor; i.e., they can be calculated to close approximation without accounting for the presence of the nozzles. In contrast, "not Quasi 1-D" means that the side branch (i.e., the nozzle) materially alters the acoustic field structure at the nozzle outlet.

Figure 19(a) provides an example of a case where the quasi 1-D approximation is quite accurate by showing the magnitude of the pressure fluctuation amplitude along a transverse line in the center plane $(y=0)$ for a $\hat{Z}_{t r} / \rho_{0} c_{0}=1$ case where the side-branch acoustics do not significantly impact the acoustics of the annulus. For reference, the solid 
curve denotes the value of the disturbance field that would exist in the absence of the nozzle. Here, the nozzle causes only a slight distortion of the disturbance field. In this case, Eq.(14) can be applied by using the acoustic pressure calculated in the absence of the nozzle.

In contrast, the nozzle significantly distorts the pressure field from the 1-D result when either $A_{c s, s} / A_{c s, A}$ is not small or $\hat{Z}_{t r} / \rho_{0} c_{0}<<1$. These two conditions are particularly important in the pressure anti-node and traveling wave cases, as shown in Figure 19(b), because the transverse mode pressure fluctuation in the region of the nozzle is significant. Little distortion occurs for the pressure node case where the nominal pressure fluctuation is zero. When the combustor acoustic field exhibits a significantly non-zero pressure near the nozzle, note how the low nozzle impedance "pulls" the pressure amplitude toward zero. In this case, the acoustic pressure fluctuation is significantly altered by the presence of the nozzle and cannot be calculated from a decoupled calculation. Further away from the nozzle in the transverse direction, the calculations that do and do not account for the presence of the side-branch converge for both the pressure anti-node and pressure node cases.

This transverse to axial flow coupling process has been highlighted in a number of studies. Staffelbach et al. [199] presented LES results from an annular combustor, clearly showing the presence of strong axial pulsations in nozzle flow accompanying transverse oscillations. This study suggested that it was these axial flow pulsations that dominated the flame response. In other words, the transverse flow oscillations serve as the "clock" that controls the natural frequency of the wave motions and the structure of the wave field, but it is the induced axial fluctuations that actually excite the heat release 
oscillations that, in turn, excite the transverse modes. Experimental observations of this same point were also made by O'Connor and Lieuwen [86], who presented a number of transversely excited flame results, see Figure 20. Figure 20(a) shows an asymmetrically forced case, where the nozzle is located at a pressure node and experiences significant transverse flow oscillations, and a relatively weak unsteady pressure field. Closer to the nozzle, however, an axial asymmetric breathing in and out of the nozzle cavity in the axial direction can be seen. This asymmetric forcing leads to the excitation of strong helical shear layer modes [195]. Figure 20(b) shows the symmetric forcing case, where the nozzle is at a pressure anti-node and acoustic velocity node. Near the nozzle, significant axisymmetric vortical velocity fluctuations are excited by bulk axial velocity fluctuations in and out of the nozzle, because of the large pressure oscillations. This leads to the strong excitation of axisymmetric shear layer modes. Instantaneous images of the flame are also indicated in Figure 20(c) and (d), showing the asymmetric and symmetric wrinkling of flames situated at pressure nodes and anti-nodes, respectively, as a result of the dominantly helical and axisymmetric shear layer modes that are excited by the forcing.

Similar flame fluctuations were seen in an annular configuration by Worth and Dawson [101, 162]. Figure 21 shows two results during $m_{a}=1$ standing-wave azimuthal oscillations. In the annular configuration, flames are not only subjected to purely symmetric (pressure anti-node) and asymmetric (velocity anti-node) disturbances, but also a range of asymmetric acoustic disturbance fields in between those nodes. The fluctuating flame response at these points around the annulus is evident in Figure 21(a) and (b), which shows the normalized fluctuating heat release rate, measured with $\mathrm{OH}^{*}$ 
chemiluminescence, at two places around the annulus at one phase of the acoustic cycle. The approximate location of the pressure anti-node is shown in Figure 21(a) where it is clear that the fluctuation in the heat release is nominally symmetric around the centerline of the nozzle, as is indicated by a similar level of fluctuation around the circumference of the flame. A flame responding asymmetrically is seen in Figure 21(b), at the velocity anti-node, where heat release rate fluctuations on either side of the flame are out of phase. Phase-averaged OH-PLIF imaging at a similar condition, shown in Figure 21(c) and Figure 21(d), shows similar flame disturbances as in Figure 20. The flame response at the pressure anti-node, in Figure 21(c), is relatively symmetric in both branches of the flame. In the image, the flame wrinkling from symmetric vortex shedding is indicated on the inner flame branch with an arrow to highlight the symmetric response of the flame. Flame response at the velocity anti-node, in Figure 21(d), clearly shows asymmetric flame response with helical flame wrinkling in both branches of the flame.

Saurabh et al. [100] also presented measurements of the response of the flame to traveling waves, showing that the flame response is similar to the flame response at the pressure anti-node in the standing wave case. In a linear framework, this result can be understood by noting that a traveling wave is the complex superposition of two standing waves with a 90 degree phase shift: $\exp (i k x)=\cos (k x)+i \sin (k x)$. As will be discussed in Section 7, the global flame response of axisymmetric flames to fluctuations at the pressure node is negligible (although the transverse to longitudinal acoustic coupling at the pressure anti-node can drive significant global heat release rate fluctuations). This would indicate that flame response to traveling wave disturbances would resemble flame response at a pressure anti-node. 


\subsubsection{Axial Temperature Inhomogeneity Effects}

In this section, we consider the effects of temperature inhomogeneity on transverse mode characteristics. We particularly focus on the case of temperature gradients in the axial direction, a situation that is very appropriate for combustor environments. For longitudinal problems, these axial temperature variations are in the same direction as the wave propagation, and so the problem remains nominally onedimensional. Natural frequencies are, to first approximation, reasonably approximated by simple time of travel arguments, i.e., scaling as $f \sim 1 /\left(2 \int d z / c(z)\right)$ for rigid boundary conditions, where $c(z)$ is the spatially varying speed of sound. In contrast, axial temperature gradients increase the dimensionality of transverse acoustic problems and modify the basic natural frequency considerations described in Sec. 5.1. In this case, the natural frequency of the system is controlled by a weighted coupling of the transverse modes in the high and low temperature/sound speed regions of the duct.

In order to illustrate how natural transverse frequencies are affected by axial temperature gradients, consider a model problem of transverse modes in a thin annular duct shown in Figure 22, neglecting fluctuations in heat release [128]. The duct is of radius $R$, where $R=\left(a_{1}+a_{2}\right) / 2$ and length, $L$, with an abrupt temperature discontinuity at $z=L_{i}$. This is a commonly used approximation for the temperature field in a combustor where the flame is acoustically compact $[80,123,125]$. Further, the axial flow is assumed to be of low Mach number axial flow, i.e. $M \square$ 1. The expressions used for the results discussed next are presented in Appendix 1, and the rest of this section presents results from this model problem. These calculations assume that the $z=0$ 
boundary is acoustically rigid and consider both rigid and pressure release boundary conditions at $z=L$.

Figure 23 shows the dependence of the normalized frequency on the azimuthal modes with $L / R=0.5$ (typical values for real hardware range from $\sim 0.5-2$ ), as a function of the temperature jump location, $\beta=L_{i} / L$, for different temperature ratios, ${ } \kappa=T_{b, 0} / T_{u, 0}$. The natural frequencies are normalized by reference frequencies based upon weighted average temperatures that are defined in the Appendix 1. A few important observations follow from these results. First, the results are reasonably captured by the reference frequency calculation based upon average temperatures, although the degree of agreement between the two is a strong function of other parameters. The parameter $L / R$ quantifies finite axial-length effects and, although not shown, the transverse results asymptote to the reference frequency in the limit of $L / R \rightarrow \infty$. This result shows that a weighted temperature approximation is a useful approximation for axial temperature variation effects. This leads to the second observation, which is that finite length effects are more important in the pressure release case. This boundary condition causes significantly more alteration of the axial wave distribution than the rigid condition, due to it forcing the unsteady pressure to zero at $z=L$. This is prominently manifested in the fact that no purely azimuthal mode is present with a pressure release boundary condition. Rather, the first azimuthal mode is a mixed axial-azimuthal mode. This is responsible for the jump in frequencies in Figure 23 between the rigid and pressure release boundary condition cases. This also shows that a purely transverse mode which might be encountered in a real engine with a choked nozzle, cannot be emulated in open ended combustor, even a full annular combustor. 


\subsubsection{Non-ideal Boundary Condition Effects}

The prior section presented an example with "perfect" boundary conditions, where the combustor inlet and outlet were rigid or pressure release. In real systems, while such a boundary condition might be a useful first approximation, there are always losses at boundaries. In this section, we consider the effect of a "nearly-rigid" and a "nearly-pressure release" boundary condition on transverse oscillations.

The "nearly-rigid" boundary condition is an important problem in low Mach number combustors with choked nozzles. The choked nozzle boundary condition has been the subject of an extensive literature for rocket applications [52, 69, 200-203]. An important follow-on to this work for axial disturbances and compact nozzles was given by Marble and Candel [204, 205], who presented a general set of results for acoustic and entropy disturbance interactions with nozzles. While the reader is referred to these references for details, the basic considerations leading to the choked nozzle boundary condition are quite straightforward in cases where the incident disturbance wavelength is long relative to the nozzle itself. In this case, there is negligible mass accumulation in the nozzle, so that the mass flow rate into the nozzle (referred to here as station $\mathrm{X}$ ), and the mass flow rate at the choke point are the same at each instant of time ${ }^{5}$. Assuming isentropic fluctuations, this equation leads to the following linearized boundary condition at station $X$ :

$$
\hat{Z}_{\text {out }}=\frac{\hat{p}_{1}}{\hat{u}_{z, 1}}=\frac{2 \rho_{0} c_{0}}{\gamma-1}\left(\frac{1}{M_{X}}\right)
$$

\footnotetext{
${ }^{5}$ Note that these relations are leading order approximations for low frequency acoustic disturbances. Stow et al. [80] also presented a higher order correction that accounts for the nozzle length and, thus, leads to a phase shift in the impedance; see Appendix 2.
} 
Equation (15) relates the pressure fluctuations to the axial flow fluctuations at the nozzle entrance point for a one dimensional problem. This equation shows that choked nozzles in low Mach number flows behave like acoustically rigid boundaries, as $\hat{Z}_{\text {out }} / \rho_{0} c_{0} \square 1$. Consider now the effect of the outlet nozzle on transverse waves. Similar reasoning suggests that pressure fluctuations present in transverse modes also lead to a mass flow modulation through the nozzle and, therefore, an indirectly induced axial flow disturbance. Stow et al. [80] showed that this same Eq.(15) can be applied to azimuthal waves in a thin gapped annulus. This implies that a transverse mode, with some azimuthal distribution of fluctuating pressure, induces an axial flow disturbance at the nozzle outlet. Similar to the discussion in Sec. 5.2.2, this leads to an axial distribution of the transverse mode amplitude and phase.

Consider open ended pipes next. In cases where the acoustic wavelength is small relative to the duct radius for circular pipes, i.e., $R / \lambda \square 1$ or $k R \square 1$, the outlet impedance for axial disturbances has been derived as an expansion in powers of $k R$, with the pressure release boundary condition (i.e. $p_{1}=0$ ) emerging as the leading order term [206]. While it is not clear whether this boundary condition requires modification for the transverse problem, it seems clear that the impedance will be similarly near zero for a pipe/annulus whose diameter/gap is small relative to the acoustic wavelength. For axial disturbances, the first order correction to approximating an open ended duct as pressure release is purely imaginary, leading to the "end correction" for the effective length of the duct, while the second order term is real, describing sound radiation (damping) from the duct. These expressions are presented in Appendix 2. 
We next illustrate quantitative results by considering finite end impedance effects. The characteristic equation for this analysis is shown in Eq.(57) of Appendix 1. In order to maximize simplicity in expressions, we will assume uniform temperature. In this case the natural modes of the duct are described by the characteristic equation:

$$
\frac{H e}{\sqrt{H e^{2}+m_{a}^{2}}} \tan \alpha H e=\frac{1}{i \tilde{\hat{Z}}_{\text {out }}}
$$

Here, $\tilde{\hat{Z}}_{\text {out }}=\hat{Z}_{\text {out }} / \rho_{b, 0} c_{b, 0}, H e=\sqrt{H e_{R}^{2}-m^{2}}$ and $H e_{R}=\omega R / c_{u, 0}$ is the dimensionless frequency, referred to as the Helmholtz number. The aspect ratio of the combustor is denoted by $\alpha=L / R$. While Eq.(16) is transcendental and can be solved computationally for arbitrary parameter values, we consider the two physically interesting limits of the choked nozzle and open outlet where $\left|\tilde{\hat{Z}}_{\text {out }}\right| \square 1$ and $\left|\tilde{\hat{Z}}_{\text {out }}\right| \square 1$. These two solutions can be expanded around the $1 / \tilde{\hat{Z}}_{\text {out }}=0$ and $\tilde{\hat{Z}}_{\text {out }}=0$ cases, respectively, whose associated eigenvalues are:

$$
\begin{aligned}
& \underset{1 / \tilde{\hat{z}}_{\text {out }}=0}{\operatorname{Rigid}} \rightarrow H e_{0}=\frac{n \pi}{\alpha} \\
& \underset{\tilde{\hat{z}}_{\text {out }}=0}{\operatorname{Pressure}} \text { Release } \rightarrow H e_{0}=\frac{(2 n-1) \pi}{2 \alpha}
\end{aligned}
$$

For the nearly rigid problem ( $\left|\tilde{\hat{Z}}_{\text {out }}\right| \square$ 1), the solutions to Eq.(16) can then be formally expanded around these values as:

$$
\left|\tilde{\hat{Z}}_{\text {out }}\right| \square 1 \rightarrow H e=H e_{0}+\frac{1}{\sqrt{\hat{Z}_{\text {out }}}} H e_{1 / 2}+\frac{1}{\hat{Z}_{\text {out }}} H e_{1}
$$

Where, 


$$
\left|\tilde{\hat{Z}}_{\text {out }}\right| \square 1 \rightarrow\left\{\begin{array}{l}
n \neq 0 \rightarrow H e_{1 / 2}=0 ; \quad H e_{1}=-\frac{i}{\alpha} \sqrt{1+\left(\frac{m_{a} \alpha}{n \pi}\right)^{2}} \\
n=0, m \neq 0 \rightarrow H e_{0}=0 ; H e_{1 / 2}=\left(\frac{1-i}{\sqrt{2}}\right) \sqrt{\frac{m_{a}}{\alpha}} ; H e_{1}=0
\end{array}\right.
$$

Note that the $\left|\hat{Z}_{\text {out }}\right| \square 1$ expansion takes two different asymptotic dependencies on $\tilde{\hat{Z}}_{\text {out }}$, depending upon whether the disturbance is an axial-transverse mode (or purely axial, i.e., $n \neq 0$ ), or a transverse mode ${ }^{6}$ (i.e., $n=0$ ); i.e., it has a $1 / \sqrt{\tilde{\hat{Z}}_{\text {out }}}$ dependence for a transverse mode and a $1 / \tilde{\hat{Z}}_{\text {out }}$ dependence for a mixed axial-transverse or pure axial mode. Thus, the spatial correction is far more significant for the purely transverse mode. However, the outlet impedance scaling of the eigenfrequency, $H e_{R}$, takes a different form. From Eq.(58) we can expand to obtain:

$$
H e_{R}^{2}-m_{a}^{2}=H e_{0}^{2}+\frac{1}{\tilde{\hat{Z}}_{\text {out }}} H e_{1 / 2}^{2}+\frac{2}{\sqrt{\tilde{\hat{Z}}_{\text {out }}}} H e_{0} H e_{1 / 2}
$$

Thus when $n=0$, we have $\left(H e_{0}=0, H e_{R, 0}=m_{a}\right)$ :

$$
2 H e_{R, 0} H e_{R, 1}=\frac{1}{\tilde{\hat{Z}}_{\text {out }}} H e_{1 / 2}^{2}
$$

This implies that even though $H e$ is $O\left(1 / \sqrt{\tilde{\hat{Z}}_{\text {out }}}\right), H e_{R}$ is $O 1 / \tilde{\hat{Z}}_{\text {out }}$.

A key difference of the eigenfrequencies for this problem from that considered in the prior sub-section is that they are not purely real numbers; the complex part of the eigenfrequency describes the exponential temporal decay of oscillations in the system

\footnotetext{
${ }^{6}$ Although even this $n=0$ mode has an axial dependence resulting from the outlet boundary condition correction
} 
due to acoustic losses at the nozzle. The axial and temporal decays can be seen explicitly by writing out the full expressions for the pressure:

$$
\begin{aligned}
& \left.n \neq 0 \rightarrow p_{1}(t, \theta, z)=\left[\begin{array}{l}
e^{i \frac{n \pi z}{L}} e^{\left\{\frac{1}{\hat{\tilde{Z}}_{\text {out }}} \sqrt{1+\left(\frac{m_{a} \alpha}{n \pi}\right)^{2}}\right\} \frac{z}{L}} \\
+e^{-i \frac{n \pi z}{L}} e^{-\left\{\frac{1}{\tilde{\hat{Z}}_{\text {out }}} \sqrt{1+\left(\frac{m_{a} \alpha}{n \pi}\right)^{2}}\right\} \frac{z}{L}}
\end{array}\right] e^{i m_{a} \theta-i\left\{\sqrt{n \pi^{2}+m_{a} \alpha^{2}}-i\right.}-\frac{i}{\hat{\tilde{Z}}_{\text {out }}}\right\} \frac{c t}{L}
\end{aligned}
$$

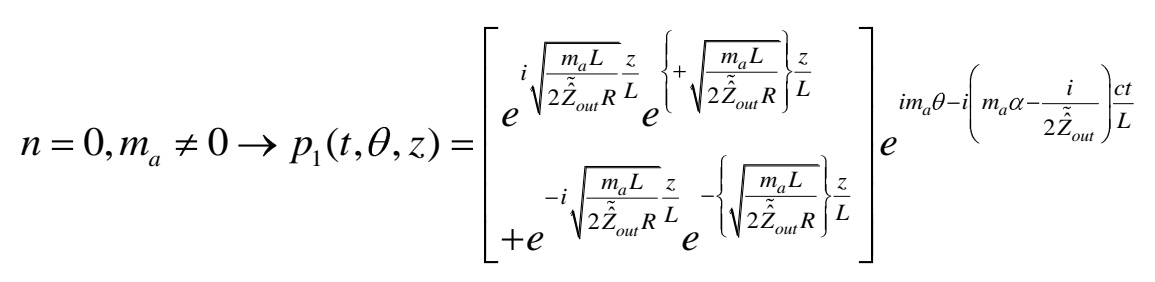

We next consider the effects of the real and imaginary parts of the impedance. For the case of purely imaginary impedance, $\tilde{\hat{Z}}_{\text {out }}$, the temporal damping coefficients for both cases are zero and the normalized frequency is:

$$
\begin{aligned}
& \alpha H e_{R}{ }_{n \neq 0}=\sqrt{n \pi^{2}+m_{a} \alpha^{2}}-\frac{1}{\operatorname{Im} \tilde{\hat{Z}}_{\text {out }}} \\
& \alpha H e_{R_{n=0}}=m_{a} \alpha-\frac{0.5}{\operatorname{Im} \tilde{\hat{Z}}_{\text {out }}}
\end{aligned}
$$

This shows that the purely transverse case shows half the frequency shift compared to the mixed mode case.

For the case of a purely real impedance, $\tilde{\hat{Z}}_{\text {out }}$, there is no frequency shift for both cases, but there is temporal damping ( $\mu$ in $\exp (-\mu c t / L))$ which is given by:

$$
\begin{aligned}
& \mu_{n \neq 0}=\frac{1}{\operatorname{Re} \tilde{\hat{Z}}_{\text {out }}} \\
& \mu_{n=0}=\frac{0.5}{\operatorname{Re} \tilde{\hat{Z}}_{\text {out }}}
\end{aligned}
$$


This shows that the damping induced on purely axial or mixed modes is twice that of purely transverse modes.

We now turn to the nearly pressure release problem $\left(\left|\hat{\mathbf{Z}}_{\text {out }}\right| \square\right.$ 1) whose solution in Eq.(16) can similarly be formally expanded as:

$$
\tilde{\hat{Z}}_{\text {out }} \square 1 \rightarrow H e_{R}=H e_{R, 0}+\tilde{\hat{Z}}_{\text {out }} H e_{R, 1}
$$

where

$$
\left|\tilde{\hat{Z}}_{\text {out }}\right| \square 1 \rightarrow H e_{R, 1}=-\frac{i}{\alpha}\left[\frac{2 n-1 \pi / 2^{2}}{2 n-1 \pi / 2^{2}+m_{a} \alpha^{2}}\right]
$$

And the associated pressure field is given by:

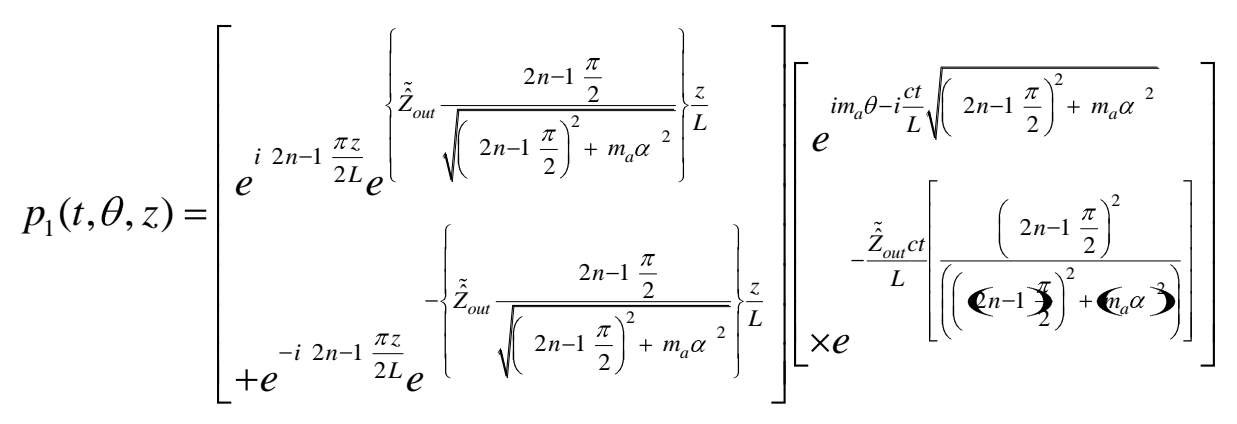

Note the purely imaginary part of the correction, showing that the nonzero impedance effect leads to acoustic damping, but no frequency shift. An important feature of this result is the fact that the damping is a function of both the axial and azimuthal mode numbers. As also discussed in Sec. 5.1, note also that there is no pure $n, m_{a}=0,1$ azimuthal mode in this case, since that would lead to a trivial solution for the pressure when enforcing the nearly pressure release outflow boundary condition. This shows that an open ended annular configuration cannot simulate the purely transverse mode that is present in combustors with choked boundary conditions. 


\section{Hydrodynamic Instabilities}

Although combustion driven oscillations are usually associated with natural acoustic frequencies that serve as the "clock" for the system frequency, the dominant source of disturbances that actually lead to heat release rate oscillations are often vortical flow disturbances. These large scale vortical disturbances themselves arise from concentration of the vorticity that largely originates from boundary layers in approach flow passages or other walls. For example, the separating free shear layer is linearly unstable due to the Kelvin-Helmholtz instability, and amplifies the perturbations that these acoustic perturbations introduce on it. At finite amplitude, these shear layer perturbations saturate into vortical structures composed of concentrated regions of vorticity [207]. The Kelvin-Helmholtz instability is present in flows with axial and azimuthal shear, while centrifugal mechanisms are also at play in swirling flows. These instabilities are also influenced strongly by the density inhomogenities in combusting flows, which both modify how vorticity induces motion on other regions through vorticity dilation processes, as well as the local vorticity through baroclinic mechanisms.

These large scale structures are embedded upon a background of broadband, smaller scale turbulence. A number of studies have used the time averaged velocity profile as inputs to laminar flow stability analyses to make predictions about flow stability behavior - such as linear disturbance amplification rates, nonlinear saturation, and disturbance frequencies [208-210]. This "quasi-laminar" approach is taken in this section as well, but we should caution that a number of questions remain around the bounds of applicability of such analyses. 
In order to fix some notation, we consider problems in both a rectangular and polar coordinate system. For nominally two-dimensional geometries, we decompose the velocity field into "symmetric" $(S)$ and "asymmetric" $(A S)$ components, often referred to as "varicose" and "sinuous" modes, respectively, as [211]:

$$
\vec{u}=\underset{\substack{\text { Symmetric } \\ \text { "Varicose" }}}{\vec{u}_{S}}+\overrightarrow{\text { Asymmetric }}_{\text {"Sinuous" }}
$$

Where the symmetric and asymmetric disturbance components are given by:

$\vec{u}_{S}(x,+y, z, t)=\vec{u}_{S}(x,-y, z, t)$

$\vec{u}_{A S}(x,+y, z, t)=-\vec{u}_{A S}(x,-y, z, t)$

and can be extracted from data using the operation:

$$
\vec{u}_{i}(x, y, z, t)=\frac{\vec{u}(x,+y, z, t)+\delta \vec{u}(x,-y, z, t)}{2} \quad\left\{\begin{aligned}
\delta=1 & \text { for } i \equiv S \\
\delta=-1 & \text { for } i \equiv A S
\end{aligned}\right.
$$

Similarly, disturbances in polar coordinates can be decomposed into modes as:

$$
\hat{u}_{i, 1} r, \varphi, z, \omega=\sum_{m_{h}=-\infty}^{\infty} \hat{B}_{i, m_{h}} r, z, \omega e^{i m_{h} \varphi}
$$

where

$$
\hat{B}_{i, m_{h}} \quad r, z, \omega=\frac{1}{2 \pi} \int_{0}^{2 \pi} e^{-i m_{h} \varphi} \hat{u}_{i, 1} \quad r, \varphi, z, \omega d \varphi
$$

Note that we use $m_{h}$ to denote hydrodynamic azimuthal mode numbers, in order to differentiate from the $m_{a}$ index used for azimuthal acoustic modes, since both disturbances will be simultaneously present. In a polar coordinate system, the $m_{h}=0$ modes describe axisymmetric disturbances and $m_{h} \neq 0$ modes describe helical disturbances. The sign of $m_{h}$, i.e., $m_{h}<0$ and $m_{h}>0$, denote counter-clockwise and 
clockwise modes, respectively ${ }^{7}$. The presence of two modes with equal amplitudes and opposite signed $m_{h}$ values denotes a "standing" wave disturbance in the azimuthal direction. A sketch of these modes in a jet is in Figure 24.

The response of the flow to external forcing can be decomposed into two subproblems, which are the focus of the following two sub-sections: (1) the flow response to a given disturbance, such as the spatial growth rate for an unstable mode, and (2) the excitation strength of the flow forcing, which is related to the initial condition. In a linear framework, each spatial mode of the flow disturbance can be expressed as:

$$
u_{i, 1}=\sum_{m_{h}=-\infty}^{m_{h}=+\infty} \hat{u}_{i, 1, m}(r, z=0) \exp -i\left(\omega t-k_{h, m} z-m_{h} \varphi\right)
$$

Where the excitation frequency, $\omega$, is a real number. The wave number for helical mode $m_{h}$ is denoted by $k_{h, m}$ and is complex, so the first sub-problem above is associated with its imaginary part, which describes the spatial growth rate of a disturbance. For example, a round jet amplifies disturbances that are both axisymmetric $\left(m_{h}=0\right)$ and helical $\left(m_{h} \neq 0\right)$ in nature, and the response of the same jet can differ markedly depending upon which of these modes are excited by the flow. This point will be discussed extensively in Sec. 7, where we show different flame response features, depending upon different acoustic forcing configurations - these different flame response features can be traced directly back to which instability modes are excited. The response of the flow to forcing

\footnotetext{
${ }^{7}$ This can be understood by considering the phase function of the disturbance at a fixed time defined as $\left(k_{h, m} z+m_{h} \varphi\right)$, where $z$ is the axial flow direction and $\varphi$ increases in the counter-clockwise direction.
} Hence, a positive mode $\left(+m_{h}\right)$ has a line of constant phase with a sense of winding in the opposite direction to $\varphi$. Conversely, a negative mode $\left(-m_{h}\right)$ is co-rotating with $\varphi$. 
is discussed first, followed by a treatment of which modes are preferentially excited by transverse forcing

\subsection{Forced Response of Hydrodynamically Unstable Flows}

A hydrodynamically unstable flow can exhibit different types of response to forcing. To understand these differences, it is useful to recall some definitions of spatial and temporal instability, as well as the concepts of convective and global instability [195, 212]. A "convectively unstable" flow region acts an amplifier, so that disturbances created at some point grow in amplitude as they convect out of the system. The growth rate of these disturbances is a function of frequency and mean flow properties, such as shear layer thickness. However, the oscillations are not self-excited, but require a continuous disturbance source to persist. During combustion instabilities, acoustic forcing at narrowband frequencies provides a strong excitation source to these spatially growing disturbances; a good example of this was shown in Figure 4.

A globally unstable flow acts as an oscillator - it is self-excited and does not require external disturbances to persist. In such a self-excited system, the amplitude grows before saturating into a limit cycle oscillation [213]. Moreover, the spectrum of oscillations shows a narrowband peak associated with this natural global mode frequency. When externally forced at another frequency, the limit cycle behavior can co-exist with the externally forced oscillations at low forcing amplitudes, and nonlinearly interact with the forcing at high forcing amplitude. For example, "lock-in" refers to a phenomenon where oscillations at the global mode frequency disappear altogether with sufficient external forcing amplitude at some other frequency. 
Constant density, non-swirling round jets are globally stable but convectively unstable. For example, in non-swirling jets, Michalke's calculations suggest that the symmetric $m_{h}=0$ mode and the first helical mode, $m_{h}= \pm 1$, should have the largest growth rates near the jet exit [214, 215], although higher order modes are also unstable. However, the relative growth rates of these different modes are a sensitive function of the jet shear layer thickness relative to the jet radius. For example, the $m_{h}=0$ and \pm 1 modes have comparable maximum growth rates for thinner boundary layer profiles.

Inviscid, constant density two-dimensional wakes are globally unstable, with the dominant mode being the sinuous one, manifesting itself as the Von Karman vortex street. Axisymmetric wakes are also globally unstable, with the first helical mode, $m_{h}= \pm 1$, having the largest temporal growth rate [216, 217]. However, the strong density gradients introduced by combustion of sufficient gas expansion ratio between reactants and products often causes the wake flow to be globally stable, so that the unsteady flow structures are dominated by the convectively unstable shear layers [218220].

Many flowfields of interest also contain swirl, and the ratio of the reference azimuthal to axial velocity, $S$, has profound influences on the flow structure. Reacting swirl flows reviews are provided by Syred [221] and Candel et al. [25] so we only summarize several key dynamical features. One of the most prominent features of high swirl number flows is the occurrence of "vortex breakdown," which is manifested as a stagnation point in the flow followed by a region of reverse flow [222]. The outer flow not entrained into the recirculating bubble accelerates around it. Typically, the flow does 
not instantaneously rotate about the geometric centerline. Rather, the point of zero azimuthal velocity is located off-center and this point itself rotates about the geometric center. This location of zero azimuthal velocity is referred to as the precessing vortex core (PVC) [223]. This global mode interacts strongly with the separating shear layers, generally causing them to roll up at the same frequency as that of the global mode. However, when forced, the convectively unstable shear layers generally respond at the forcing frequency, resulting in the periodic formation of vortices in the shear layers that convect downstream with the mean flow. However, the central vortex breakdown region structure is altered only at high forcing levels, consistent with its globally unstable nature [87]. Swirl causes important influences on spatial growth rates of shear layer disturbances. In particular, it biases the strength of co- and counter-winding helical vortices, so that positive and negative mode numbers do not have the same growth rate.

Figure 25 shows an example of a linear spatial growth rate calculation, plotting the exponential growth rate, i.e., the imaginary part of $k_{h, m}$, of several modes as a function of excitation frequency for a swirling jet. The different growth rates of the $m_{h}=-1$ and $m_{h}=+1$ modes are due to the presence of swirl. Note how the growth rate is a strong function of both frequency and which spatial mode is being considered.

\subsection{Excitation Features of Transversely Forced Flows}

As shown above, a given flow has multiple unstable modes that have different spatial mode shapes. Thus, not only the frequency, but also the spatial dependence of the forcing strongly influences the response of the flow. This controls the initial amplitude of the disturbance, the second sub-problem described above Eq.(34). 
During longitudinal acoustic instabilities, the acoustic field provides a strong symmetric forcing of the flow field - thus, it preferentially excites the varicose $\delta=1$ and axisymmetric $\left(m_{h}=0\right)$ mode in the two-dimensional and axisymmetric cases, respectively. In contrast, transverse oscillations generally excite non-symmetric modes. To illustrate, consider the decomposition of a disturbance into transverse modes, using the coordinate system shown in Figure 26. Note that the polar coordinate system used here (polar angle is $\varphi$ ) is centered on a given nozzle, and is different from the azimuthal coordinate system (azimuthal angle $\theta$ ) used for the entire annular combustor (such as in Figure 22).

Consider first a one-dimensional acoustic disturbance of the form $\exp (i k x)$, such as might be encountered in an annular combustor. A traveling wave $e^{i k x}$ (or, if considering waves in an annular combustion chamber, the traveling wave is given by $e^{i k R \theta}$ ) can be equivalently decomposed as:

$$
e^{i k x}=\sum_{m_{h}=-\infty}^{\infty} \hat{C}_{m_{h}}(k r) e^{i m_{h} \varphi}
$$

where the strength of azimuthal mode $m$ can be determined using the Jacobi-Anger expansion as:

$$
\text { "traveling wave forcing" } \rightarrow e^{i k x} \Rightarrow \hat{C}_{m_{h}}(k r)=i^{m_{h}} J_{m_{h}}(k r)
$$

and $J_{m_{h}}$ denotes the Bessel function of the first kind. This expression shows that a single traveling wave in the $x$ direction in the rectangular coordinate system can be written as a superposition of an infinite sum of helical disturbances in the polar coordinate system - it thus shows which helical disturbances are most strongly excited by such a traveling 
wave. The results for the modal amplitude strengths, $\hat{C}_{m_{h}}$, for the asymmetric and symmetric forcing can similarly be derived and are shown in Table 3.

In many problems of interest, the nozzle/flame region is small relative to an acoustic wavelength, so $k r \square 1$. Expanding the Bessel functions in a Taylor series and retaining only the leading order term leads to a result that is more easily interpretable and is also shown in the table. These results can be summarized in three key points. First, traveling waves most strongly excite the $m_{h}=0$ modes, but also other even and odd modes. Next, symmetric forcing most strongly excites the axisymmetric, $m_{h}=0$ mode, and does not excite odd modes. Finally, asymmetric forcing most strongly excites the $m_{h}= \pm 1$ modes, and does not excite even modes.

Note in all cases how the amplitude of higher modes drops off quickly in the $k r<<1$ case. The dependence of these coefficients upon mode number are plotted in Figure 27(a) and (b), which can be thought of as a plot of the strength of the forcing of the different hydrodynamic modes of the flow. It can be hypothesized that hydrodynamic modes that are more strongly forced by the acoustic field will manifest themselves with higher responses. While the relative sensitivities of the hydrodynamic modes to excitation also comes into play as described in Sec. 6.1, this hypothesis is generally the case as illustrated in Figure 27(c) and (d), which plots measured velocity fluctuation amplitudes from O'Connor and Lieuwen [87] - these are the hydrodynamic responses to acoustic forcing. Note the very close correspondence of the dominant measured modes, to those that the decomposition shows to be excited by the method of forcing. The one noticeable difference between the experimental results and the analytical symmetric and 
asymmetric prediction is the strong response at $m_{h}=-2$ in the experimental symmetric forcing case. This is likely a result of hydrodynamic amplification of this mode as a result of the natural instability characteristics of this flow field; measurements of the unforced flow have shown that $m_{h}=-2$ is a preferred mode [87]. This result clearly shows how both predictions and measurements bear out the influence of the excitation field structure on the dominant hydrodynamic response. These results complement those shown in Figure 20, and illustrate the fundamental difference that nozzle placement in an acoustic standing wave field plays out in terms of excited hydrodynamic disturbances.

\subsection{Transverse forcing of typical combustor flow fields}

With this background, the rest of this section reviews studies that have analyzed the forced response of flows to transverse (or non-axisymmetric) forcing. Studies of shear layer instability and forced response include forcing the flow from the upstream plenum [224, 225], by mechanical actuation [226, 227], or transversely deflecting the shear layer [228-234]. For example, Fiedler and Mensing [233] forced the shear layer transversely by exciting a waveguide that opened at a slit at the separation point of the mixing layer. The authors noted that a key distinction from bulk-flow forcing was that the boundary layer is periodically "deflected" by the transverse mass flow fluctuation at the slit. A theoretical effort by Tam [234] explored the role of an incident sound wave's angle of incidence relative to the shear layer, showing that an angle between $50^{\circ}$ and $80^{\circ}$ was the most effective at driving the shear layer instability. The efficacy of the forced response was also found to be a function of the acoustic beam half-width, the acoustic frequency, the shear layer thickness, and the Mach number of the flow. 
Studies of the transverse forcing of wakes typically fall into two categories: those looking at the re-attachment of wakes on bluff-bodies [235-237], and the control of wakes of airfoils [238-242]. Re-attachment studies often use slits at the shear layer separation point, similar to Fiedler and Mensing [233], to control shear layer development. The free-stream forcing of airfoil wakes indicates that transverse forcing of the wake or a separation region can significantly reduce the size of the separation bubble, as well as the intensity and size of coherent structures shedding from the back of the airfoil.

A number of studies have investigated the response of jets to transverse forcing. Some of these studies focus on shear layer control and use excitation at the separation point [243-250], mechanical actuation [248, 251-253], or transverse excitation of the free stream by both standing waves [254] and traveling waves [237, 255-257]. Experiments show that control of the shear layer structures, jet turbulence levels, and jet spreading rate is readily achievable with non-axisymmetric forcing. For example, Kusek et al. [250] used twelve speakers located around the jet exit to excite azimuthal modes in the circular jet. Figure 28 shows an example of the smoke visualization from this experiment, illustrating the considerable effect that non-axisymmetric forcing can have on flow response. In Figure 28(a), a symmetric forcing function is imposed on the convectively unstable jet, resulting in a symmetric response, whereas in Figure 28(b), an asymmetric forcing function drives an asymmetric response from the jet. An extreme example of the effects of non-axisymmetric response of jets is in the case of bifurcating and blooming jets [258]. 
Finally, a growing number of studies have investigated the impact of transverse acoustic forcing on swirling flows [86, 87, 259-261]. Studies used acoustic forcing at the jet separation point [259-261], and in the cases of Gallaire et al. [260] and Panda and McLaughlin [261], the azimuthal distribution of the forcing was controlled, as in Kusek et al. [250] Similar results to those from Kusek et al. [250] were found in the swirling flow studies, indicating that the response of the convectively unstable shear layers mirrored the symmetry of the input forcing function. Work by O'Connor and Lieuwen [88] corroborated these shear layer results in the jet near-field, but also showed a growth in the $m_{h}=-1$ mode as a function of downstream distance, even when the external forcing function was symmetric; this is believed to be a result of the natural instability characteristics of the jet shear layer being superimposed on the shear layer response to the forcing function. Results from Gursul [259] and O'Connor and Lieuwen [87] showed that the vortex breakdown region of the flow field, which stems from a global instability, responds very differently to transverse excitation than the shear layers. Gursul observed "lock-in" of the helical instability in the vortex breakdown region downstream of the jet exit, while O'Connor and Lieuwen did not observe any dynamical response of the PVC to transverse forcing. However, O'Connor and Lieuwen did observe a large-scale bifurcation in the vortex breakdown structure at high amplitudes of forcing, somewhat similar to the "blooming jet" phenomenon observed in asymmetrically forced jets.

In two phase flows, transverse acoustic forcing of liquid jets [262-264], supercritical jets $[59,60,63,64,265-268]$, and droplets [269, 270] has shown that the development of interfacial instabilities can be significantly altered by transverse acoustic fields. In liquid jets, the transverse forcing acts to displace the liquid jet and changes the 
jet breakup characteristics at a range of Weber numbers [262]. This problem has been studied extensively in rocket applications, where transverse oscillations are the dominant form of combustion instability. For example, imaging by Talley and coworkers $[59,60$, 265] of transversely forced sub- and supercritical jets has shown that the acoustic field periodically displaces the jet, and can change the time-average spreading rate of the jet, and hence its entrainment rate. An example of these results is shown in Figure 29, where a subcritical co-axial jet is subjected to transverse acoustic forcing [271]. In each of the images, the phase angle in the top-right of the image indicates the phase between the speakers on either end of the forcing facility. " 0 deg" leads to a pressure anti-node at the jet, where " 180 deg" creates a pressure node. Comparison of the response of the liquid jet at each forcing condition with the "no acoustics" case shows a number of the ways that transverse acoustics impacts liquid jet breakup. First, there is significant lateral displacement of the jet, particularly near the " $180 \mathrm{deg}$," or velocity anti-node, case where local velocity fluctuations drive large-scale motion of the jet core. As a result of this, the jet spreading angle near the velocity anti-node/pressure node is much greater. Finally, acoustic driving also changes the length of the "dark core," or the continuous liquid core of the jet, accelerating atomization.

In addition, the induced axial air flow fluctuations described in Sec. 5.2.1 also influence the spray. Studies of axial forcing of nonreacting sprays into an atmospheric pressure ambient have shown that atomization and spray spreading characteristics are modulated [272-274]. For example, Eckstein et al. [272] concluded that the instantaneous Sauter mean diameter was modulated by axial forcing, but in a quasisteady manner. In other words, utilizing the instantaneous air flow velocity in steady 
state droplet sizing correlations captured the instantaneous droplets sizes. In addition, the spray cone angle can be modulated by forcing, such as through modulation of the flow swirl number [273].

\section{Flame Response}

This section discusses more fully the sensitivity of the flame to transverse instabilities. Starting with transverse flow and pressure disturbances as the input, and heat release oscillations as the output, there are multiple pathways through which heat release oscillations are excited, as shown in Figure 30. The flame is dominantly sensitive to flow disturbances (referred to as "velocity coupling"), as well as any fuel/air ratio oscillations excited by these disturbances ("fuel/air ratio coupling"), while its direct sensitivity to pressure fluctuations ("pressure coupling") is weak relative to these other

sources in low Mach number flows ${ }^{8}$ [195]. The pathways for longitudinal oscillations are present in this figure because of the importance of transverse to longitudinal coupling [76].

\subsection{Overview of Flame Excitation Processes}

Four difference sources of heat release rate fluctuations, or flame response, are shown in Figure 30. The net flame response is a complex summation of the transfer functions from these above sources, $F_{\phi}, F_{L}, F_{\omega}, F_{T}$, multiplied by the disturbance source strength associated with each process.

\footnotetext{
${ }^{8}$ Note however, that while the flame itself may not be very sensitive to pressure disturbances, axial flow oscillations and fuel/air ratio oscillations may exhibit very important pressure sensitivities. Thus, it is important to be precise with what ones means by "pressure -coupling"
} 
First, the flame can be directly excited by the transverse acoustic field, and the flame transfer function $F_{T}$ (defined in Eq.(6)) describes the magnitude and phase of the flame response to transverse acoustic velocity fluctuations. Acoustic coupling between the transverse-mode fluctuations in the combustor and longitudinal motion in the nozzle, referred to as "injector coupling," is described by the velocity transfer function $F_{T L}$. The resulting longitudinal acoustic fluctuations also excite the flame, either through the velocity coupled mechanism, $F_{L}$, or by exciting fuel/air ratio oscillations, $F_{\phi}$.

Fuel/air ratio oscillations are excited in both gaseous and two- phase flows. For example, axial acoustic disturbances in premixed systems lead to a time varying air mass flow rate that the fuel is mixing with. In two-phase flows, acoustic fluctuations in both transverse and axial directions modulate spray breakup, atomization, and vaporization processes. These fuel/air ratio fluctuations are induced by both modulation of droplet sizes (discussed in Sec.6.23) [274], as well as modulation of the spray cone angle, moving the location of the fuel droplets with respect to the air [273]. This pathway is described by the transfer function $F_{\omega \phi}$. However, it is important to be precise about the actual physical processes that result in heat release oscillation. In premixed systems, the actual fuel/air ratio of the mixture reacted at the flame is modulated [275]. In nonpremixed systems, downstream mixing could similarly lead to modulation of mixture stoichiometry, but could also simply reflect modulation of some more global fuel/air ratio, with the actual mode of burning remaining nonpremixed with a local equivalence ratio of unity.

Returning to the acoustic-to-flow instability transfer functions, $F_{T \omega}$ and $F_{L \omega}$, the vortical velocity fluctuations that result from this coupling can also lead to flame 
response. Such flame forcing by vortical disturbances is shown in Figure 20 and Figure 21 , and described by the flame transfer function $F_{\omega}$. We will consider the influence of these vortical disturbances on the flame further in this section.

\subsection{Connection to Rayleigh Criterion: Role of Characteristic Times}

Whether or not these pathways cause heat release fluctuations that add energy to the acoustic field depends upon the phasing of the unsteady heat release with respect to the pressure, following Eq.(1). It is often more physically intuitive to equate the phase between pressure and heat release, given by $\Phi$, to a characteristic time delay between them, $\tau_{q}$, where the two are related by:

$$
\Phi=\frac{2 \pi \tau_{q}}{T_{a}}
$$

where $T_{a}$ denotes the acoustic period. The flame adds energy to the acoustic field when:

$$
\Phi<\frac{\pi}{2} \rightarrow-\frac{1}{4}<\frac{\tau_{q}}{T_{a}}<\frac{1}{4}
$$

This time delay, $\tau_{q}$, is itself the sum of all the intermediate delays associated with the progression from pressure disturbances to heat release. This is illustrated in Figure 31, which shows how acoustic pressure disturbances propagate through the system and excite different disturbances that lead to heat release oscillations - results are shown for three of the different pathways in Figure 30. This plot clearly shows how the phase of the transfer functions between the different steps or, equivalently, their time delays, control the conditions under which the resultant heat release rate oscillations are in phase with the pressure. For example, the middle plot shows how pressure disturbances in a transverse disturbance lead to fuel/air ratio disturbances and, consequently, heat release rate 
oscillations. In this case, the pressure and heat release rate oscillations are in phase when the sum of the characteristic delay times shown in the plot add up to an integer multiple of $T_{a}$, as expressed in Eq.(38).

\subsection{Response of Flames to Direct Transverse Flow Excitation $\left(F_{T}\right.$ pathway)}

In order to understand the relative strength of the different coupling pathways shown in the diagram in Figure 30, it is useful to note two key attributes of transverse excitation that differentiate it from axial disturbances. First, while longitudinal disturbances are generally axisymmetric, or at least approximately so, transverse disturbances excite the flame in an intrinsically non-axisymmetric manner that causes significant azimuthal variations in local disturbance field at the flame sheet. As shown in Eq.(36) and Table 3, transverse disturbances excite both axisymmetric and helical/asymmetric disturbances in the flow, while axial disturbances excite $m_{h}=0$ /symmetric flow disturbances. Second, while the flame does not locally differentiate between different vector components of the velocity (being disturbed by the scalar velocity component normal to it, as can be seen by manipulation of Eqs.(2) and (3)), transverse and axial excitation cause profoundly different influences upon the flame heat release oscillations when considered more globally. In the low frequency, quasi-steady limit, the instantaneous heat release is directly proportional to the instantaneous mass flow rate of reactants into the flame [276]. As shown in Figure 32(a), axial disturbances modulate the mass flow rate of reactants into the domain for both premixed and nonpremixed flames and, as such, the low frequency transfer function for both flames 
asymptotes to $F=1[171,181,276]$; in other words, a 5\% flow velocity disturbance leads to a 5\% fluctuation in heat release rate. In contrast, Figure 32(b) shows that purely transverse disturbances do not modulate the reactant mass flow rate into the flame and, as such, the low frequency flame transfer function asymptotes to zero for both premixed and nonpremixed flames $[127,180]$. Thus, bulk transverse flow disturbances can lead to significant wrinkling and flapping of the flame, but no heat release oscillations.

Transverse flow disturbances lead to reactant mass flow modulation through the flame, as in Figure 32(b), only if the flux through the left and right flame branches are not the same (such as if the center of the flame is a velocity node), but this effect is relatively weak since the transverse velocity fluctuations will necessarily be weak near the node, unless the flame width is on the order of an acoustic wavelength.

For this reason, the direct response of the flame to transverse flow disturbances, the $F_{T}$ path in Figure 30, is quite weak, as also indicated in Figure 31.

\subsection{Response of Flames to Axial Flow Excitation ( $F_{L}$ pathway)}

We consider next the various longitudinal forcing pathways shown in Figure 30. Response of the flame to longitudinal acoustic fluctuations can be quite significant. A significant body of well-validated theory has been developed for modeling the response of laminar flames to velocity disturbances using the flamelet approaches described in Sec. 4.2. For example, the transfer function of a premixed flame excited by spatially uniform axial disturbances is $[32,173,277]$ : 


$$
\begin{aligned}
& F_{L}=2\left[\frac{i S t-1-i S t e^{i S t}}{S t^{2}}\right] \\
& \text { where, } S t=\frac{\omega L_{f}}{u_{0}}\left(\frac{1+\chi^{2}}{\chi^{2}}\right) \text { and } \chi=2 L_{f} / D
\end{aligned}
$$

Here, $S t$ is the Strouhal number and $\chi$ is the flame aspect ratio. Transfer functions of this form can then be used to describe the response of the flame to bulk axial disturbances induced by transverse waves described in Sec. 5 .

\subsection{Response of Flames to Vortical Disturbances $\left(F_{T \omega}\right.$ and $\left.F_{L \omega}\right)$}

We next consider the flame response to hydrodynamic flow disturbances, such as convecting vortices that are included in the $F_{\omega}$ path in Figure 30. Assuming a polar coordinate system, the spatially integrated unsteady heat release rate, shown in Eq.(4), is rewritten as:

$$
\dot{Q}=\iint_{A} \dot{m}_{F}^{\prime \prime}(r, \theta, t) h_{R}(r, \theta, t) d A_{f}(r, \theta, t)
$$

Expanding each quantity as a sum of its steady $\quad_{0}$ and unsteady components ${ }_{1}$ and considering the Fourier domain representation of the unsteady heat release fluctuations, we have:

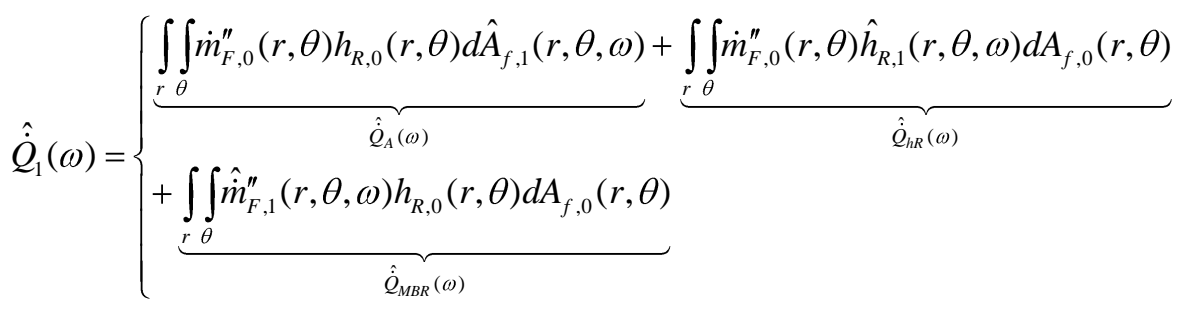

Each of the fluctuating variables can be expanded in azimuthal components as:

$$
{ }_{1}(r, \varphi, \omega)=\sum_{m_{h}=-\infty}^{\infty}{ }_{1, m_{h}}(r, \omega) e^{i m_{h} \varphi}
$$


We can derive a very significant result for a general, axisymmetric flame from this expression. For cases where all time averaged variables are axisymmetric, i.e., ${ }_{0} \neq f n(\varphi)$, they can be pulled out of the angular integral. Take, for example, the last term in Eq.(41), which can then be written as:

$$
\hat{\dot{Q}}_{M B R}(\omega)=\int_{r}\left(\sum_{m_{h}=-\infty}^{\infty}\left[\hat{\dot{m}}_{F, 1, m_{h}}^{\prime \prime}(r, \omega) h_{R, 0}(r) d A_{f, 0}(r)\right] \int_{\varphi=0}^{2 \pi} e^{i m_{h} \varphi} d \varphi\right)
$$

For non-axisymmetric modes $\left(m_{h} \neq 0\right)$, the integral over $\varphi$ is zero (i.e., $\left.\int_{\varphi=0}^{2 \pi} e^{i m_{h} \varphi} d \varphi=0\right)$, which implies that only the axisymmetric $m_{h}=0$ mode contributes to the global unsteady heat release rate fluctuations. In other words:

$$
\hat{\dot{Q}}_{M B R}(\omega)=\left\{\begin{array}{cc}
2 \pi \int\left[\left(\hat{\dot{m}}_{F, m_{h}=0}^{\prime \prime}\right)^{\prime}(r, \omega) \bar{h}_{R}(r)\right] d A_{f, 0}(r) & m_{h}=0 \\
0 & m_{h} \neq 0
\end{array}\right.
$$

An important implication of these results is that helical modes $m_{h} \neq 0$, while introducing substantial wrinkling of the flame front, do not lead to fluctuations in the azimuthally integrated heat release in axisymmetric flames. Only if the time averaged flame position is not axisymmetric do helical modes lead to net heat release oscillations. Furthermore, while the above analysis has been carried out for flamelets, similar reasoning would apply to any distributed combustion zone as well. Thus, while transverse acoustic disturbances, as well as the helical hydrodynamic disturbances that they excite (e.g., see Table 3), can directly lead to significant flame flapping, the heat release rate oscillations they excite may be quite small. Rather it is the induced axial 
acoustic disturbances, as well as the symmetric or $m_{h}=0$ hydrodynamic disturbances that are excited, that dominate the flame response.

For this reason, we consider further the response of flame to $m_{h}=0$ disturbances. The transfer function of a premixed flame, excited by an axisymmetric disturbance convecting at a speed of $u_{c}$ is [32]:

$$
\begin{aligned}
& F_{\omega, m_{h}=0}=2\left[\frac{\eta-1+i \quad i+S t \quad \eta e^{i S t}+1-\eta S t e^{i \eta S t}}{\eta 1-\eta S t^{2}}\right] \\
& \text { where, } \quad \eta=\frac{u_{0}}{u_{c}}\left(\frac{\chi^{2}}{1+\chi^{2}}\right)
\end{aligned}
$$

While this simple result has been developed for a laminar flame with spatially uniform mean velocity, more detailed calculations that better represent the spatial variation of the mean velocity field, and time averaged turbulent flame characteristics show quite good agreement with measurement flame responses [93, 128].

The effect of helical instabilities on flame dynamics has been investigated by several researchers $[33,76,278,279]$ and support the notion that excitation by symmetric disturbances dominate flame response. For example, flame transfer functions measurements from transversely forced flames show that the response of flames located at pressure anti-nodes is stronger than those at nodes [76, 96, 179], in agreement with the above discussion. For example, Figure 33 plots measured flame transfer functions from Acharya et al. [128], obtained from the facility as shown in Figure 8b. Note the stronger response of the flame in the symmetric forcing case than the asymmetric forcing case for a given velocity disturbance. 
However, helical modes can lead to globally integrated heat release oscillations if the nominal flame shape is non axisymmetric. These non-axisymmetries can and do arise through, for example, confinement or flame-flame interactions, such as shown in Figure 11. In such cases, the asymmetric helical modes in the disturbance flow-field lead to non-zero FTF contributions [129]. Thus, in instances where helical modes are much stronger than axisymmetric ones (e.g., see the data in Figure 27), the degree of flame non-axisymmetry can determine which disturbance pathway in Figure 30 is dominant. This result also shows that flame response measurements taken from single flame configurations in circular geometries, where the time averaged flame will be axisymmetric, can differ from the response of flames located in non-circular geometries or in multi-nozzle configurations with significant flame-flame interactions. The former geometry will effectively filter out the effect of strong helical modes that may be present on the flame response, while flames in the latter cases will have sensitivity to these disturbances. This illustrates another challenge in scaling transverse instability conditions with single nozzle configurations that do not correctly emulate the flame shape.

\section{System Thermoacoustic Instability Model Problem}

Having considered different elements of the problem, we now present results from a simplified, quasi one-dimensional model that combines many of the problem's key physical elements into an overall thermoacoustic stability analysis of azimuthal modes. This analysis follows the presentations by Stow et al. [80] and Parmentier et al. [123], and will consider azimuthal oscillations in a thin annular combustor, ignoring plenum acoustics (see, e.g., Bauerheim et al. [79], for inclusion of plenum effects). For 
simplicity, we assume the nozzle flow upstream of the combustion chamber is isothermal with properties given by the subscript $(u)$, and the combustor is isothermal at the burned gas temperature, given by the subscript $(b)$ (thus, assuming a short flame at the nozzle outlet). We also neglect mean flow effects or entropy modes (associated with entropy fluctuations incident upon nozzles that excite acoustic waves).

The basic geometry is shown in Figure 2, which consists of $N$ nozzles mounted at an azimuthal spacing $d_{s}$, and identified with the index $s=1 \ldots N$. Note that $\sum_{s=1}^{N} d_{s}=2 \pi R$. Within a chamber between nozzle $s-1$ and $s$, the acoustic field is a one-dimensional function of the azimuthal direction and given by:

$$
\begin{aligned}
& \hat{p}_{1, s}^{b}(\theta)=G_{+, s}^{b} \exp \left(i k_{b} R \theta\right)+G_{-, s}^{b} \exp \left(-i k_{b} R \theta\right) \\
& \hat{u}_{\theta, 1, s}^{b}(\theta)=\frac{1}{\rho_{b, 0} c_{b, 0}} G_{+, s}^{b} \exp \left(i k_{b} R \theta\right)-G_{-, s}^{b} \exp \left(-i k_{b} R \theta\right)
\end{aligned}
$$

Here, $k_{b}=\omega / c_{b, 0}$ and $\theta=0$ starts at nozzle $s-1$ and ends at nozzle $s$ such that: $0 \leq \theta \leq d_{s} / R$. Thus, for each nozzle, there are 2 unknowns, $\mathscr{G}_{+, s}^{b}$ and $\mathscr{G}_{-, s}^{b}$, leading to a total of $2 N$ unknowns for the entire annular combustor. These coefficients are solved for by solving momentum and energy relations for a control volume encompassing the nozzle region, as shown in Figure 34.

The nozzle-combustion chamber intersection is treated using acoustically compact T-junction relations, which lead to the following matching conditions for acoustic pressure and velocity at the interfaces $[17,280]$ :

$$
\text { Momentum: } \quad \hat{p}_{1, s}^{u}=\hat{p}_{1, s}^{b}\left(\theta=d_{s} / R\right)=\hat{p}_{1, s+1}^{b}(\theta=0)
$$

Energy: $A_{c s, A} \hat{u}_{\theta, 1, s+1}^{b}(\theta=0)=A_{c s, A} \hat{u}_{\theta, 1, s}^{b}\left(\theta=d_{s} / R\right)+A_{c s, s} \hat{u}_{z, 1, s}^{u}\left[1+\hat{F}_{s}(\omega)\right]$ 
The derivation of Eq.(48) is presented in Appendix 3. Here, $A_{c s, A}$ is the cross-sectional area of an axial-radial cut of the combustor and $A_{c s, s}$ is the cross-sectional area of nozzle $s$. In addition, it is assumed that the unsteady heat release equals the axial acoustic flow velocity at the nozzle outlet multiplied by the transfer function $\hat{F}_{s} \omega$. This transfer function incorporates the response of the flame to both axial acoustic flow disturbances and the axisymmetric vortical disturbances they excite, given in Eq.(39) and Eq.(45), respectively. However, this model could be easily generalized to include sensitivity of the flame to transverse disturbances, such as might occur when transverse disturbances excite helical modes that interact with a non-axisymmetric flame.

At the nozzle outlet into the annulus, and for frequencies well below the nozzle cutoff frequency (for which transverse modes are possible in a nozzle itself), the axial nozzle outlet velocity is related to the pressure through the translated nozzle impedance as shown in Eq. (13):

$$
\hat{p}_{1, s}^{u}=-\hat{Z}_{t r} \hat{u}_{z, 1, s}^{u}
$$

Note that the negative sign results from the fact that the impedance is defined as the velocity into the nozzle outlet boundary. For a single nozzle of length $h$, terminated upstream by an impedance, $\hat{Z}_{o}$, the expression for $\hat{Z}_{t r}$ is given by Eq. (13). More generally, it describes the influence of all upstream elements associated with each nozzle.

For the $N$ nozzles, Eqs.(47)-(49) can be combined into a $2 N$ by $2 N$ matrix. This matrix can be simplified to a 2 by 2 matrix whose determinant is the characteristic equation: 


$$
\operatorname{det} .\left(\prod_{s=1}^{N} \mathscr{T}_{s}-\left[\begin{array}{ll}
1 & 0 \\
0 & 1
\end{array}\right]\right)=0
$$

Where:

$$
\mathscr{\mathscr { T } _ { s }}=\left[\begin{array}{cc}
e^{i k_{b} d_{s}} & 0 \\
0 & e^{-i k_{b} d_{s}}
\end{array}\right]+\mathscr{O}\left[\begin{array}{cc}
e^{i k_{b} d_{s}} & e^{-i k_{b} d_{s}} \\
-e^{i k_{b} d_{s}} & -e^{-i k_{b} d_{s}}
\end{array}\right]
$$

And, $\mathscr{O}_{s}$ is a "nozzle coupling factor" given by:

$$
\mathscr{G}=-\frac{1}{2} \frac{A_{c s, s} / A_{c s, A}}{Z_{t r} / \rho_{b, 0} c_{b, 0}}\left[1+\hat{F}_{s}(\omega)\right]
$$

In Appendix 4, Eq. (50) has been expanded using Eq. (51) to derive the characteristic equation for the case of all nozzles having identical geometric and flame properties $\left(\mathscr{G} \equiv, \hat{F}_{s} \equiv \hat{F}\right.$ ). For the following discussion, we shall express the flame transfer function as: $\hat{F} \omega=n \exp -i \omega \tau_{q}$ where $n$ and $\tau_{q}$ are the frequency independent gain and time-delay, respectively, discussed previously in the context of Eqs.(6)-(7). From Eq.(72) and Eq.(73) in the Appendix, we can extract the imaginary part of the frequency, corresponding to the exponential growth/decay rate of disturbances. While a general solution can be calculated with computational methods, explicit analytical results are derived next for the case where $\square 1$ (or) $\square 1$ and $\hat{Z}_{t r}$ is independent of frequency ${ }^{9}$. For $\square$ 1, we obtain: $\operatorname{Im}\left(\frac{\omega R}{c_{b, 0}}\right)=-\frac{N}{2 \pi} \frac{A_{c s, s}}{A_{c s, A}} \frac{\left[\begin{array}{lll}1+n \cos 2 \pi m_{a} \tilde{\tau}_{q} & \operatorname{Re} \tilde{\hat{Z}}_{t r}-n \sin 2 \pi m_{a} \tilde{\tau}_{q} \operatorname{Im} \tilde{\hat{Z}}_{t r}\end{array}\right]}{\left|\tilde{\hat{Z}}_{t r}\right|^{2}}$

\footnotetext{
${ }^{9}$ While assuming $\hat{Z}_{t r}$ is independent of the frequency is a useful assumption for pedagogical purposes of showing explicit results, in reality $\hat{Z}_{t r}$ is a function of frequency as shown in Eq.(13)
} 
For $\square 1$ it is given by:

$\operatorname{Im}\left(\frac{\omega R}{c_{b, 0}}\right)=-\frac{2 N}{\pi} \frac{A_{c s, s}}{A_{c s, A}} \frac{\left[\begin{array}{llll}1+n \cos 2 \pi m_{a} \tilde{\tau}_{q} & \operatorname{Re} \tilde{\hat{Z}}_{t r}-n \sin 2 \pi m_{a} \tilde{\tau}_{q} \operatorname{Im} \tilde{\hat{Z}}_{t r}\end{array}\right]}{\sqrt{1+2 n \cos 2 \pi n_{a} \tilde{\tau}_{q}+n^{2}}}$

Here, $\tilde{\hat{Z}}_{t r}=\hat{Z}_{t r} / \rho_{b, 0} c_{b, 0}$ and $\tilde{\tau}_{q}=\tau_{q} / 2 \pi R / c_{b, 0}$ such that a value of $\tilde{\tau}_{q}=1$ corresponds to a normalized time delay equal to one acoustic period of the first azimuthal mode $m_{a}=1$ in the absence of unsteady heat release. Note that Eq.(54) can be further simplified for small values of $n$ (or low amplification of the disturbance by the flame):

$$
\operatorname{Im}\left(\frac{\omega R}{c_{b, 0}}\right)=-\frac{2 N}{\pi} \frac{A_{c s, s}}{A_{c s, A}}\left[\operatorname{Re} \tilde{\hat{Z}}_{t r}-n \sin 2 \pi n_{a} \tilde{\tau}_{q} \operatorname{Im} \tilde{\hat{Z}}_{t r}\right]
$$

Several observations can be gained from these expressions. First, the instability growth/decay rate is a function of the four parameters: $n, m_{a}, \tilde{\tau}_{q}$ and $\tilde{\hat{Z}}_{t r}$. The two quantities $N$ and $A_{c s, s} / A_{c s, A}$ only scale the growth/decay rate value, however, and do not influence stability boundaries. Second, in the absence of heat release, $n=0$, these expressions show that $\operatorname{Im} \omega<0$ when $\operatorname{Re} \tilde{\hat{Z}}_{t r}>0$ and, thus, that oscillations are damped. This is due to the finite (though small) damping imposed by $\operatorname{Re} \tilde{\hat{Z}}_{t r}$ at the inflow nozzles (note that outlet nozzle damping is not incorporated in this simplified model). Third, the instability growth/decay rate is directly proportional to the number of nozzles, $N$, the nozzle/combustor area ratio (reflecting the dependence of the unsteady heat release upon the axial disturbance velocity), and either $\tilde{\hat{Z}}_{t r}$ or $1 / \tilde{\hat{Z}}_{t r}$ (for $\tilde{\hat{Z}}_{t r} \square 1$ and $\tilde{\hat{Z}}_{t r} \square 1$, respectively). This latter $\tilde{\hat{Z}}_{t r}$ dependence shows that the instability 
growth/decay rate is small for both of these cases. For the $\tilde{\hat{Z}}_{t r} \square 1$ case, the axial nozzle velocity is very small, leading to low amplitude heat release oscillations. For the $\tilde{\hat{Z}}_{t r} \square 1$ case, the acoustic pressure at the flame is weak, leading to low Rayleigh gains (see Eq.(1) ) .

Finally, $\operatorname{Im} \omega$ has an oscillatory dependence upon $\tilde{\tau}_{q}$. This shows the effect of the heat release time delay upon stability limits, due to the influence of this time delay parameter upon the pressure-heat release phase. For this plots, it is convenient to define a normalized frequency given by:

$$
\tilde{\omega}=\left(\frac{\omega R}{N c_{b, 0}}\right)\left(\frac{A_{c s, A}}{A_{c s, s}}\right)
$$

The variation of $\operatorname{Im} \tilde{\omega}$ for the 1 case is plotted in Figure 35 for both $m_{a}=1$ and $m_{a}=2$. As anticipated, the growth rate is an oscillatory function of $\tilde{\tau}_{q}$, and oscillates between positive and negative values. Such oscillatory dependencies upon this dimensionless time-lag can be inferred from Figure 31 and have been well documented in axial instability studies, such as summarized in the results from Chapter 6 of Lieuwen [17].

\section{Concluding Remarks and future work}

Transverse modes are a dominant mode of combustion instability in a range of combustion devices, and the challenges associated with studying and controlling these modes are arguably greater than those associated with longitudinal instabilities. 
Prominent observations from this review are organized next under the headings of acoustics, hydrodynamics, and flame response:

- Acoustics:

- In annular combustion systems with large area ratios between nozzles and the combustor cross sectional area, $A_{c s, s} / A_{c s, A} \square 1$, the transverse mode acts as the "clock", setting the frequency and spatial structure of the acoustic forcing field. However, it is the axial acoustic disturbances through the nozzle, and the vortical disturbances that they excite, that often dominate the flame response.

- The sensitivity of the axial flow oscillations in the inflow nozzles to transverse forcing are a function of the relative location of the nozzle within the acoustic disturbance field (e.g., a pressure node vs. anti-node), as well as the upstream acoustic nozzle impedance. This nozzle impedance is governed by burner aerodynamics and plenum acoustics.

- Boundary conditions have significant influences on transverse modes. First, it is not possible to have a purely azimuthal or radial mode for an open-ended (pressure release) boundary condition only mixed axial-transverse modes are possible. In addition, choked nozzles cause lower acoustic losses to transverse modes than axial ones. 
Transverse motions in upstream plenums, though not discussed extensively here, introduce additional degrees of freedom to the problem.

- Hydrodynamics:

- High shear combustor flows are hydrodynamically unstable and sensitive to acoustic perturbations. The response of the hydrodynamic instabilities to acoustic perturbation depends upon the flows convective/absolute instability character, the acoustic frequency, and acoustic mode shape.

- The same frequency acoustic disturbance can excite different hydrodynamic modes (e.g., axisymmetric vs. helical modes in jets), depending upon the location of the shear flow relative to the acoustic disturbance field (e.g., acoustic pressure node vs. antinode).

- Flame response:

- The flame's spatial integrated heat release is nearly insensitive to direct transverse flow disturbances. However, it is quite sensitive to axial acoustic disturbances, as well as axisymmetric hydrodynamic disturbances.

$\circ$ The heat release rate of axisymmetric flames is insensitive to helical flow disturbances. Thus, in flows with strong helical modes, the degree of axisymmetry of the unforced flame (due to, for example, flame-flame interactions or confinement effects) will profoundly influence its flame response. 
Issues and challenges associated with simulating or scaling instabilities have been discussed throughout the review. Key observations are:

- Sub-scale arrangements are more challenging for simulating the acoustic transverse mode shapes than for longitudinal instabilities, as the transverse scale of the combustor, not just its length must be emulated. The degree to which this can be done is often limited by air flow rate.

- Transverse modes in circular geometries support traveling waves, something which cannot be simulated in sector geometries or rectangular geometries (where, in contrast, the axial acoustic mode shapes in circular and sector/rectangular geometries can be quite close to the full scale system), without specialized forcing at the transverse boundaries.

- Combustors with open (unrestricted) ends do not have a purely transverse or radial mode, a major problem if one is attempting to simulate the first tangential or radial mode of a combustor.

- In many instances, the key flame response in acoustically compact cases can be simulated with axial instabilities, assuming that the nozzle impedance and unforced flame shape is correctly simulated.

- While the flame response during an axial instability may be comparable to that of a transverse instability, the combustor damping is not, as the acoustic damping provided by a choked nozzle for a transverse disturbance is half that of an axial one. 
Future work to further understand and predict the behavior of transverse combustion instabilities falls into several categories. First, continued monitoring of instabilities in operational combustion systems and determination of the conditions under which transverse instabilities appear is a valuable asset to the scientific and engineering community. In particular, there is little open literature on can combustion systems. While data for operational systems is usually proprietary, means for disseminating general observations are important for enabling the research community to mobilize around key problems. Next, capturing the correct acoustic boundary conditions and mode shapes has been shown to be a first-order importance in determining the behavior of an unstable combustor. The following research items address some of the needs to better quantify the acoustic processes that occur during a combustion instability.

- Inlet nozzle impedance and its dependence upon key parameters is critical to understanding the extent of transverse to longitudinal coupling. For example, acoustic calculations show a strong sensitivity of the axial velocity induced by a transverse wave [195] to often ignored properties such as fillet radii of the outlet (note that an angle discontinuity excites an infinite amplitude acoustic field in an inviscid framework). While viscous effects and the excitation of vorticity (which can be modeled by incorporating the Kutta condition [281]), will smooth out this singularity, better understanding of how nozzle parameters influence transverse to longitudinal acoustic coupling is needed.

- The analysis in this review focused its attention on cases where the inlet nozzle is acoustically compact to the transverse acoustic mode. However, frameworks are needed for describing problems in cases where this 
assumption is not appropriate, such as in can-combustors, or during radial modes in annular combustors.

- Similarly, the nozzle cross-sectional area may not be small as compared to the combustor cross-sectional area; this situation is found regularly in can combustors or for radial modes in annular combustors. While this situation can be modeled without difficulty with 3-D Helmholtz solvers or LES, reduced order modeling frameworks are needed for interpretation of results, such as the transverse to longitudinal coupling process.

Some particular needs for better understanding the hydrodynamic response of the flow field to transverse acoustic fields are.

- Hydrodynamic stability characteristics of reacting swirl flows: While a large and growing literature on this topic exists, the generality of these results is major challenge. It is likely that several general classes of problems exist (e.g., those dominated by shear instability mechanisms vs those dominated by centrifugal ones, or those where the flame lies inside or outside the shear layer) and a "taxonomy" for classifying and interpreting swirl flow results is needed. Such a taxonomy might include general parameterizations on axial and azimuthal shear profiles, density profiles, and Reynolds number.

- Forced response of the convectively unstable flow: This receptivity problem is indicated by by $F_{T \omega}$ and $F_{L \omega}$ in Figure 30 . Local stability analysis results, such as absolute growth rate and spatial growth rate, can exhibit different parametric sensitivities. A simple example is the strong sensitivity of the 
absolute growth rate of a reacting wake to flame density ratio, but the much weaker sensitivity of the spatial growth rate of the forced flow problem.

- Forced response of globally unstable flows: interaction between nominal flow dynamics and the forcing is intrinsically nonlinear and bifurcations and hysteresis in properties such as time averaged flow features are often observed.

- Response of two-phase flows to external forcing, including influence on droplet sizes, oscillatory spray location, fuel/air ratio and mixedness distributions. Current models are largely phenomenological and further insight is needed for development of models and interpretive frameworks.

A limited number of studies have investigated the detailed flame response to transverse instabilities, either on a local or global scale. To this end, the follow issues should be addressed in order to better understand flame response to transverse acoustic excitation and the role it plays in driving the combustion instability.

- Measurements of the response of flames to helical modes are needed where the axisymmetry of the flame can be systematically varied.

- Flame response to mixed transverse and longitudinal modes, as well as instabilities with both spinning and standing waves.

- How flame feedback influences the preference to spinning versus standing modes.

Finally, work on active control of combustion instabilities is needed. While active control has been demonstrated on transverse instabilities, generally there is no $a$ priori way of determining how effective a given system will be. Reported values for the 
degree of suppression of instabilities varies substantially in the literature [282], and deeper understanding of the factors that influence how effective a given device will be are needed.

\section{Acknowledgements}

Vishal Acharya and Timothy Lieuwen acknowledge support from the US Department of Energy contract DE-NT0005054 (contract monitor Mark Freeman) and from the National Science Foundation contract CBET-1235779 (contract monitor Professor Ruey-Hung Chen). Jacqueline O'Connor gratefully acknowledges the support of the College of Engineering and the Department of Mechanical and Nuclear Engineering at the Pennsylvania State University. The authors also gratefully acknowledge many helpful comments and critiques of this manuscript offered by Dr. Michael Bauerheim, Prof. James Dawson, Prof. Matthew Juniper, Prof. Nicolas Noiray, Prof. Thierry Schuller, and Prof. Thierry Poinsot, as well as the anonymous reviewers. 


\section{Appendix 1}

In the thin annular gap approximation, the acoustic pressure and axial velocity in each region (Figure 22) are given by the expressions [17]:

$$
\begin{aligned}
& \hat{p}_{1}(z, \theta)=\left[\mathscr{A}_{1} \exp i k_{z, n} z+\mathscr{A}_{2} \exp -i k_{z, n} z\right]\left[\mathscr{G}_{1} \exp (i m \theta)+\mathscr{G}_{2} \exp (-i m \theta)\right] \\
& \hat{u}_{z, 1}(z, \theta)=\frac{k_{z, n}}{\omega \rho_{0}}\left[\mathscr{A}_{1} \exp i k_{z, n} z-\mathscr{A}_{2} \exp -i k_{z, n} z\right]\left[\mathscr{G}_{1} \exp (i m \theta)+\mathscr{C}_{2} \exp (-i m \theta)\right]
\end{aligned}
$$

The upstream inlet boundary $(z=0)$ is considered to be rigid and the temperature jump interface is located at $z=L_{i}$. The mean properties in the regions before and after the interface (denoted by subscript $i$ ) are:

$$
\begin{aligned}
& 0<z<L_{i} \rightarrow \rho_{u, 0}, T_{u, 0} \\
& L_{i}<z<L \rightarrow \rho_{b, 0}, T_{b, 0}
\end{aligned}
$$

Although not necessary, for simplicity we will assume that the molecular weights and mean pressure are the same in the two regions, so that $\rho_{b, 0} / \rho_{u, 0}=T_{u, 0} / T_{b, 0}$ and $c_{b, 0} / c_{u, 0}=\sqrt{T_{b, 0} / T_{u, 0}}$. At the rigid upstream boundary $\left(1 / \hat{Z}_{o}=0\right)$, we have:

$$
\hat{u}_{z, 1}(z=0, \theta)=0
$$

For the impedance condition at $z=L$ :

$$
\hat{p}_{1}(z=L, \theta)=\hat{Z}_{\text {out }} \hat{u}_{z, 1}(z=L, \theta)
$$

At the interface, pressure and axial velocity are matched at each azimuthal location:

$$
\begin{aligned}
& \hat{p}_{1}\left(z=L_{i}^{-}, \theta\right)=\hat{p}_{1}\left(z=L_{i}^{+}, \theta\right) \\
& \hat{u}_{z, 1}\left(z=L_{i}^{-}, \theta\right)=\hat{u}_{z, 1}\left(z=L_{i}^{+}, \theta\right)
\end{aligned}
$$


Note that transverse modes excite vortical and entropy disturbances, whose amplitudes are determined by matching transverse velocities and mass flow rate at the interface, respectively; however, the effect of these vortical and entropy disturbances on the acoustic modes are a lower order effect, of $O M$ and $O M^{2}$, respectively, and are not calculated for space reasons (for example, see Ref. [283] for the effect of vorticity waves on azimuthal instabilities). Thus, using Eqs.(58)-(60) with Eq.(57) results in the dispersion relation:

$$
\left.\begin{array}{l}
\sqrt{\kappa} H e_{\kappa} \cos \alpha \beta H e\left[\cos \left(\frac{\alpha 1-\beta}{\sqrt{\kappa}} H e_{\kappa}\right)-i \hat{Z}_{\text {out }} \frac{H e_{\kappa}}{H e_{R}} \sin \left(\frac{\alpha 1-\beta}{\sqrt{\kappa}} H e_{\kappa}\right)\right] \\
-H e \sin \alpha \beta H e\left[\sin \left(\frac{\alpha 1-\beta}{\sqrt{\kappa}} H e_{\kappa}\right)+i \tilde{\hat{Z}}_{\text {out }} \frac{H e_{\kappa}}{H e_{R}} \cos \left(\frac{\alpha 1-\beta}{\sqrt{\kappa}} H e_{\kappa}\right)\right]
\end{array}\right\}=0
$$

Here:

$$
\begin{aligned}
& H e_{R}=\omega R / c_{u, 0} \\
& H e=\sqrt{H e_{R}^{2}-m^{2}} \\
& H e_{\kappa}=\sqrt{H e_{R}^{2}-m^{2} \kappa}
\end{aligned}
$$

And, the parameters $\alpha, \beta, \kappa$ and $\hat{Z}_{\text {out }}$ are defined as:

$$
\begin{array}{lr}
\alpha=L / R & \beta=L_{i} / L \\
\kappa=T_{b, 0} / T_{u, 0} & \hat{\tilde{Z}}_{\text {out }}=\hat{Z}_{\text {out }} / \rho_{b, 0} c_{b, 0}
\end{array}
$$

Consdier the rigid $\left(\hat{Z}_{\text {out }} \rightarrow \infty\right)$ and pressure release $\left(\hat{Z}_{\text {out }}=0\right)$ combustor exit boundary conditions, respectively. As discussed in Sec. 5.1, these are good approximations for combustors with open and choked outlets. We can define two reference frequencies for the axial and transverse modes that are based on time of travel arguments. A reference 
frequency for the purely axial modes corresponds to the time of travel back and forth for a wave across the distance $L$ through the two regions of differing sound speed:

$$
\begin{aligned}
& \text { Rigid } \rightarrow f_{\text {ref }, \text { axial }}=\frac{n}{2 L\left(\frac{\beta}{c_{u, 0}}+\frac{1-\beta}{c_{b, 0}}\right)} \\
& \text { Pressure Release } \rightarrow f_{\text {ref }, \text { axial }}=\frac{2 n-1}{4 L\left(\frac{\beta}{c_{u, 0}}+\frac{1-\beta}{c_{b, 0}}\right)}
\end{aligned}
$$

where $\beta=L_{i} / L$.

Similarly, a reference frequency for the transverse modes can be defined based upon the weighted natural frequencies of the two regions:

$$
f_{\text {ref, transverse }}=m_{a} \frac{c_{u, 0} \beta+c_{b, 0} 1-\beta}{2 \pi R}
$$

\section{Appendix 2}

Higher order correction to the choked boundary condition result is given by [80]:

$$
2 \frac{u_{z, 1}}{c_{0}}-\frac{p_{1}}{p_{0}}+\frac{\rho_{1}}{\rho_{0}}=\frac{i k l}{1-U^{2}}\left[\begin{array}{l}
\left(\frac{1}{\gamma} \frac{p_{1}}{p_{0}}+\frac{\rho_{1}}{\rho_{0}}+2 m_{a} \frac{u_{\theta, 1}}{c_{0}}\right)^{z / l} \int_{0}^{1} \frac{1}{U(z)} d z \\
-\left(\frac{\gamma-1}{\gamma} \frac{p_{1}}{p_{0}}\right)_{0}^{z / l} U(z) d z
\end{array}\right]+O(k l)^{2}
$$

Here, $U(z)=u_{z, 0}(z) / c_{0}^{*}$.

Similarly, higher order corrections for open ended pipes responding to axial flow disturbances are given by the following expressions [206]:

$$
\text { Flanged: } \quad \hat{Z}_{\text {out }}=\frac{\hat{Z}}{\rho_{0} c_{0}}=i \frac{8}{3 \pi} k R+\frac{1}{2} k R^{2}+O k R^{3}
$$




$$
\text { Unflanged: } \quad \hat{Z}_{\text {out }}=0.61 i k R+\frac{1}{4} k R^{2}+O k R^{3}
$$

\section{Appendix 3}

The energy balance in the control volume ( $C V_{s}$ in Figure 34$)$ leads to the expression [280]:

$$
A_{c s, A} \hat{u}_{\theta, 1, s+1}^{b}(\theta=0)=A_{c s, A} \hat{u}_{\theta, 1, s}^{b}\left(\theta=d_{s} / R\right)+A_{c s, s} \hat{u}_{z, 1, s}^{b}
$$

The quantity $\hat{u}_{z, 1, s}^{b}$ denotes the unsteady velocity that stems from both the axial inflow at the nozzle exit into the control volume and the presence of heat release from the flame. This is given by:

$$
A_{c s, s} \hat{u}_{z, 1, s}^{b}=A_{c s, s} \hat{u}_{z, 1, s}^{u}+\frac{\gamma_{u}-1}{\gamma_{u} p_{0}} \hat{\dot{q}}_{1, s}
$$

Following the results from Sec. 4.2, the unsteady heat release at the $s$-th injector is assumed to be sensitive to the axial flow at the nozzle outlet through the transfer function:

$$
\frac{\gamma_{u}-1}{\gamma_{u} p_{0}} \hat{\dot{q}}_{1, s}=A_{c s, s} \hat{F}_{s}(\omega) \hat{u}_{z, 1, s}^{u}
$$

Thus, combining Eqs.(69)-(71), we obtain Eq.(48).

\section{Appendix 4}

Assuming all the nozzles to be the same, we have $\equiv$ and drop the subscript $s$. Using Eq.(51), Eq.(50) becomes: 


$$
\begin{aligned}
& 8 \mathscr{O} e^{i 4 \pi H e_{N}}\left[1-\mathscr{O}+1+\mathscr{O} e^{i 4 \pi H e_{N}}\right]^{2}-4 e^{i 4 \pi H e_{N}} \\
& \otimes\left(\left[\begin{array}{c}
1-\mathscr{O}+1+\mathscr{O} e^{i 4 \pi H e_{N}} \\
\left.-\sqrt{\mathscr{O}-1^{2}+\mathscr{O}+1^{2} e^{i 8 \pi H e_{N}}-21+\mathscr{O}^{2} e^{i 4 \pi H e_{N}}}\right]^{N}-2 e^{i 4 \pi H e_{N}}{ }^{N}
\end{array}\right]^{N}-2 e^{i 4 \pi H e_{N}}{ }^{N}\right)=0 \\
& \otimes\left(\left[\begin{array}{c}
1-\mathscr{O}+1+\mathscr{O} e^{i 4 \pi H e_{N}} \\
\left.+\sqrt{\mathscr{O}-1^{2}+\mathscr{O}+1^{2} e^{i 8 \pi H e_{N}}-21+\mathscr{O}^{2} e^{i 4 \pi H e_{N}}}\right]^{N}
\end{array}\right.\right.
\end{aligned}
$$

Here,

$$
\begin{aligned}
& H e_{N}=H e / N \\
& H e=\omega R / c_{b, 0}
\end{aligned}
$$

Note that $=\mathrm{He}$ as defined in Eq.(52).

This can be reduced to 3 equations. The first equation is:

$$
\left[1-\mathscr{G}+1+\mathscr{\mathscr { C }} e^{i 4 \pi H e_{N}}\right]^{2}-4 e^{i 4 \pi H e_{N}}=0
$$

Which can be re-written as:

$$
\frac{H e}{N}=m_{a}-\frac{i}{2 \pi} \ln \left[\frac{1-\mathscr{O}(H e)}{1+\mathscr{O}(H e)}\right]
$$

Here, $m_{a}$ is a positive integer corresponding to the azimuthal mode number. For fixed values of the nozzle geometry and the flame parameters, depends on $\mathrm{He}$ and $\hat{Z}_{t r}$. For the limiting case of $\hat{Z}_{t r}=0$ and $1 / \hat{Z}_{t r}=0$, these is no growth or damping in the system and this results in $\mathrm{He} / \mathrm{N}=1$. For weak dependencies of $\mathscr{O}(\mathrm{He})$ on $\mathrm{He}$, which typically occur for $\square 1$ and $\square$ 1, we can derive asymptotic results from Eq.(75): 


$$
\begin{aligned}
& \square 1 \rightarrow \frac{H e}{N}=m_{a}+\frac{i \mathscr{G}}{\pi}+\frac{i \mathscr{G}^{3}}{3 \pi}+O \\
& 1 \rightarrow \frac{H e}{N}=m_{a}+\frac{i}{\pi}+\frac{i}{3 \pi \mathscr{C}^{3}}+O\left(\frac{1}{\mathscr{G}^{4}}\right)
\end{aligned}
$$

Note, for the asymptotic cases,

$$
\begin{aligned}
& \mathscr{F}=-\frac{1}{2} \frac{A_{c s, s} / A_{c s, A}}{\hat{Z}_{t r} / \rho_{b, 0} c_{b, 0}}\left[1+n \cos 2 \pi n_{a} \tilde{\tau}_{q}-i n \sin 2 \pi n_{a} \tilde{\tau}_{q}\right] \\
& \frac{1}{\mathscr{G}}=-2 \frac{\hat{Z}_{t r} / \rho_{b, 0} c_{b, 0}}{A_{c s, s} / A_{c s, A}}\left[\frac{1+n \cos 2 \pi n_{a} \tilde{\tau}_{q}+i n \sin 2 \pi n_{a} \tilde{\tau}_{q}}{\sqrt{1+2 n \cos 2 \pi n_{a} \tilde{\tau}_{q}+n^{2}}}\right]
\end{aligned}
$$

Where, $n$ is the amplitude of the $F T F$ and $\tilde{\tau}_{q}=\tau_{q} / 2 \pi R / c_{b, 0}$ is the non-dimensional time-lag in the FTF model. The second and third equations are:

$$
\begin{aligned}
& {\left[\begin{array}{l}
1-\mathscr{G}+1+e^{i 4 \pi H e_{N}} \\
-\sqrt{-1^{2}+\mathscr{O}+1^{2} e^{i 8 \pi H e_{N}}-21+\mathscr{C}^{2} e^{i 4 \pi H e_{N}}}
\end{array}\right]^{N}-2 e^{i 4 \pi H e_{N}{ }^{N}}=0} \\
& {\left[\begin{array}{l}
1-\mathscr{O}+1+e^{i 4 \pi H e_{N}} \\
+\sqrt{-1^{2}+1^{2} e^{i 8 \pi H e_{N}}-21+\mathscr{C}^{2} e^{i 4 \pi H e_{N}}}
\end{array}\right]^{N}-2 e^{i 4 \pi H e_{N}{ }^{N}}=0}
\end{aligned}
$$

These equations result in either purely imaginary solutions ( $\mathrm{Re} H e=0$ ) or solutions which are governed by Eq.(75). 


\section{References}

1. Lieuwen, T.C., Yang, V., and Lu, F.K., Combustion Instabilities in Gas Turbine Engines: Operational Experience, Fundamental Mechanisms and Modeling. 2005: American Institute of Aeronautics and Astronautics.

2. Mongia, H.C., Held, T.J., Hsiao, G.C., and Pandalai, R.P., Incorporation of Combustion Instability Issues into Design Process: Ge Aeroderivative and Aero Engines Experience, in Combustion Instabilities in Gas Turbine Engines, T.C. Lieuwen and V. Yang, Editors. 2005, AIAA: Washington D.C.

3. Cohen, J., Hagen, G., Banaszuk, A., and Becz, S., Attenuation of Combustor Pressure Oscillations Using Symmetry Breaking, in 49th AIAA Aerospace Sciences Meeting2011: Orlando, FL.

4. Smith, K. and Blust, J., Combustion Instabilities in Industrial Gas Turbines: Solar Turbines' Experience, in Combustion Instabilities in Gas Turbine Engines, T.C. Lieuwen and V. Yang, Editors. 2005, AIAA: Washington D.C. p. 29-42.

5. Krebs, W., Bethke, S., Lepers, J., Flohr, P., and Prade, B., Thermoacoustic Design Tools and Passive Control: Siemens Power Generation Approaches, in Combustion Instabilities in Gas Turbine Engines, T.C. Lieuwen and V. Yang, Editors. 2005, AIAA: Washington D.C. p. 89-112.

6. Zellhuber, M., Meraner, C., Kulkarni, R., Polifke, W., and Schuermans, B., Large Eddy Simulation of Flame Response to Transverse Acoustic Excitation in a Model Reheat Combustor. Journal of engineering for gas turbines and power, 2013. 135(9).

7. Sewell, J. and Sobieski, P., Monitoring of Combustion Instabilities: Calpine's Experience, in Combustion Instabilities in Gas Turbine Engines, T.C. Lieuwen and V. Yang, Editors. 2005, AIAA: Washington D.C. p. 147-162.

8. Ebrahimi, H.B. Overview of Gas Turbine Augmentor Design, Operation, and Combustion Oscillation. in 42nd AIAA/ASME/SAE/ASEE Joint Propulsion Conference. 2006. Sacramento, CA.

9. $\quad$ Rogers, D.E. and Marble, F.E., A Mechanism for High-Frequency Oscillation in Ramjet Combustors and Afterburners. Journal of Jet Propulsion, 1956. 26(6): p. 456-462.

10. Elias, I., Acoustical Resonances Produced by Combustion of a Fuel-Air Mixture in a Rectangular Duct. The Journal of the Acoustical Society of America, 1959. 31: p. 296.

11. Kaskan, W.E. and Noreen, A.E., High-Frequency Oscillations of a Flame Held by a Bluff Body. Transactions of the ASME, 1955: p. 885-895.

12. King, C.R., Experimental Investigation of Effects of Combustion Chamber Length and Inlet Total Temperature, Total Pressure, and Velocity on Afterburner Performance, in NACA Research Memorandum1957, NACA.

13. Staff, L.L., A Summary of Preliminary Investigations into the Characteristics of Combustion Screech in Ducted Burners, 1958, NACA. 
14. Macquisten, M.A. and Dowling, A.P., Combustion Oscillations in a Twin-Stream Afterburner. Journal of Sound and Vibration, 1995. 188(4): p. 545-560.

15. Rayleigh, J.W.S.B., The Theory of Sound. Vol. 2. 1896: Macmillan.

16. Brear, M., Nicoud, F., Talei, M., Giauque, A., and Hawkes, E., Disturbance Energy Transport and Sound Production in Gaseous Combustion. Journal of Fluid Mechanics, 2012. 707: p. 53-73.

17. Lieuwen, T.C., Unsteady Combustor Physics. 2012: Cambridge University Press.

18. Dowling, A., Thermoacoustic Sources and Instabilities, in Modern Methods in Analytical Acoustics: Lecture Notes, D. Crighton, A. Dowling, Ffowcs Williams JE, M. Heckl, and F. Leppington, Editors. 1992, Springer-Verlag: New York. p. 378-405.

19. Hughes, I. and Dowling, A., The Absorption of Sound by Perforated Linings. Journal of Fluid Mechanics, 1990. 218: p. 299-335.

20. Balachandran, R., Ayoola, B.O., Kaminski, C.F., Dowling, A.P., and Mastorakos, E., Experimental Investigation of the Nonlinear Response of Turbulent Premixed Flames to Imposed Inlet Velocity Oscillations. Combustion and Flame, 2005. 143(1-2): p. 37-55.

21. Cohen, J. and Banaszuk, A., Factors Affecting the Control of Unstable Combustors, in Combustion Instabilities in Gas Turbine Engines, T. Lieuwen and V. Yang, Editors. 2005, AIAA: Washington, D.C.

22. McManus, K., Poinsot, T., and Candel, S., A Review of Active Control of Combustion Instabilities. Progress in energy and combustion science, 1993. 19(1): p. 1-29.

23. Ducruix, S., Schuller, T., Durox, D., and Candel, S., Combustion Dynamics and Instabilities: Elementary Coupling and Driving Mechanisms. Journal of Propulsion and Power, 2003. 19(5): p. 722-734.

24. Huang, Y. and Yang, V., Dynamics and Stability of Lean-Premixed SwirlStabilized Combustion. Progress in Energy and Combustion Science, 2009. 35(4): p. 293-364.

25. Candel, S., Durox, D., Schuller, T., Bourgouin, J.-F., and Moeck, J.P., Dynamics of Swirling Flames. Annual Review of Fluid Mechanics, 2014. 46(1): p. 147-173.

26. Venkataraman, K., Preston, L., Simons, D., Lee, B., Lee, J., and Santavicca, D., Mechanism of Combustion Instability in a Lean Premixed Dump Combustor. Journal of Propulsion and Power, 1999. 15(6): p. 909-918.

27. Shreekrishna, Response Mechanisms of Attached Premixed Flames Subjected to Harmonic Forcing, in Aerospace Engineering2011, Georgia Institute of Technology: Atlanta, GA.

28. Poinsot, T.J., Trouve, A.C., Veynante, D.P., Candel, S.M., and Esposito, E.J., Vortex-Driven Acoustically Coupled Combustion Instabilities. Journal of Fluid Mechanics, 1987. 177: p. 265-292.

29. Schadow, K., Gutmark, E., Parr, T., Parr, D., Wilson, K., and Crump, J., LargeScale Coherent Structures as Drivers of Combustion Instability. Combustion science and technology, 1989. 64(4-6): p. 167-186.

30. Thumuluru, S.K. and Lieuwen, T., Characterization of Acoustically Forced Swirl Flame Dynamics. Proceedings of the Combustion Institute, 2009. 32(2): p. 28932900. 
31. Palies, P., Durox, D., Schuller, T., and Candel, S., The Combined Dynamics of Swirler and Turbulent Premixed Swirling Flames. Combustion and Flame, 2010. 157(9): p. 1698-1717.

32. Preetham, Hemchandra, S., and Lieuwen, T., Dynamics of Laminar Premixed Flames Forced by Harmonic Velocity Disturbances. Journal of Propulsion and Power, 2008. 24(6): p. 1390-1402.

33. Paschereit, C.O., Gutmark, E., and Weisenstein, W., Coherent Structures in Swirling Flows and Their Role in Acoustic Combustion Control. Physics of Fluids (1994-present), 1999. 11(9): p. 2667-2678.

34. Balachandran, R., Ayoola, B., Kaminski, C., Dowling, A., and Mastorakos, E., Experimental Investigation of the Nonlinear Response of Turbulent Premixed Flames to Imposed Inlet Velocity Oscillations. Combustion and Flame, 2005. 143(1): p. 37-55.

35. Huang, Y. and Yang, V., Effect of Swirl on Combustion Dynamics in a LeanPremixed Swirl-Stabilized Combustor. Proceedings of the Combustion Institute, 2005. 30(2): p. 1775-1782.

36. Sattelmayer, T., Influence of the Combustor Aerodynamics on Combustion Instabilities from Equivalence Ratio Fluctuations. Journal of Engineering for Gas Turbines and Power, 2003. 125(1): p. 11-19.

37. Lee, J.G., Kim, K., and Santavicca, D., Measurement of Equivalence Ratio Fluctuation and Its Effect on Heat Release During Unstable Combustion. Proceedings of the Combustion Institute, 2000. 28(1): p. 415-421.

38. Lieuwen, T. and Zinn, B.T. The Role of Equivalence Ratio Oscillations in Driving Combustion Instabilities in Low Nox Gas Turbines. in Symposium (International) on Combustion. 1998. Elsevier.

39. Shreekrishna, Hemchandra, S., and Lieuwen, T., Premixed Flame Response to Equivalence Ratio Perturbations. Combustion Theory and Modelling, 2010. 14(5): p. 681-714.

40. Birbaud, A.-L., Ducruix, S., Durox, D., and Candel, S., The Nonlinear Response of Inverted " $V$ " Flames to Equivalence Ratio Nonuniformities. Combustion and Flame, 2008. 154(3): p. 356-367.

41. Murat Altay, H., Speth, R.L., Hudgins, D.E., and Ghoniem, A.F., The Impact of Equivalence Ratio Oscillations on Combustion Dynamics in a Backward-Facing Step Combustor. Combustion and Flame, 2009. 156(11): p. 2106-2116.

42. McIntosh, A., The Interaction of High Frequency Low Amplitude Acoustic Waves with Premixed Flames, in Nonlinear Waves in Active Media. 1989, Springer. p. 218-231.

43. Ledder, G. and KAPHA, A., The Response of Premixed Flames to Pressure Perturbations. Combustion science and technology, 1991. 76(1-3): p. 21-44.

44. Keller, D. and Peters, N., Transient Pressure Effects in the Evolution Equation for Premixed Flame Fronts. Theoretical and Computational Fluid Dynamics, 1994. 6(2-3): p. 141-159.

45. Clavin, P., Pelcé, P., and He, L., One-Dimensional Vibratory Instability of Planar Flames Propagating in Tubes. Journal of Fluid Mechanics, 1990. 216: p. 299-322. 
46. Wangher, A., Searby, G., and Quinard, J., Experimental Investigation of the Unsteady Response of Premixed Flame Fronts to Acoustic Pressure Waves. Combustion and Flame, 2008. 154(1): p. 310-318.

47. Schmidt, H. and JIMenez, C., Numerical Study of the Direct Pressure Effect of Acoustic Waves in Planar Premixed Flames. Combustion and flame, 2010. 157(8): p. 1610-1619.

48. Sirignano, W., Delplanque, J., Chiang, C., and Bhatia, R., Liquid-Propellant Droplet Vaporization: A Rate-Controlling Process for Combustion Instability. Liquid rocket engine combustion instability(A 96-11301 01-20), Washington, DC, American Institute of Aeronautics and Astronautics, Inc.(Progress in Astronautics and Aeronautics., 1995. 169: p. 307-343.

49. Duvvur, A., Chiang, C., and Sirignano, W., Oscillatory Fuel Droplet Vaporization-Driving Mechanism for Combustion Instability. Journal of propulsion and Power, 1996. 12(2): p. 358-365.

50. Rosner, D.E. and Chang, W., Transient Evaporation and Combustion of a Fuel Droplet near Its Critical Temperature. Combustion Science and Technology, 1973. 7(4): p. 145-158.

51. Yang, V. and Anderson, W., Liquid Rocket Engine Combustion Instability. 1995, Washington D.C.: AIAA.

52. Crocco, L., Harrje, D.T., Sirignano, W.A., Bracco, F.V., Mitchell, C.E., Tang, P.K., Williams, R.M., Black, G.R., and Stinger, W.A., Nonlinear Aspects of Combustion Instability in Liquid Propellant Rocket Motors, 1967, NASA.

53. Harrje, D.T. and Reardon, F.H., Liquid Propellant Rocket Combustion Instability, 1972, NASA.

54. Dranovsky, M.L., Combustion Instabilities in Liquid Rocket Engines: Testing and Development Practices in Russia. Progress in Aeronautics and Astronautics. 2007, Washington D.C.: AIAA.

55. Richecoeur, F., Ducruix, S., Scouflaire, P., and Candel, S., Effect of Temperature Fluctuations on High Frequency Acoustic Coupling. Proceedings of the Combustion Institute, 2009. 32(2): p. 1663-1670.

56. Richecoeur, F., Ducruix, S., Scouflaire, P., and Candel, S., Experimental Investigation of High-Frequency Combustion Instabilities in Liquid Rocket Engine. Acta Astronautica, 2008. 62(1): p. 18-27.

57. Quinlan, J., Bibik, O., and Zinn, B., Experimental and Numerical Combustion Instability Modes in Asymmetric Baffled Chambers, in 46th AIAA/ASME/SAE/ASEE Joint Propulsion Conference 2010: Nashville, TN.

58. Hardi, J., Oschwald, M., and Dally, B., Flame Response to Acoustic Excitation in a Rectangular Rocket Combustor with Lox/H2 Propellants. CEAS Space Journal, 2011. 2(1-4): p. 41-49.

59. Davis, D., Chehroudi, B., Talley, D., Engineering, R., and Consulting Inc Edwards Afb, C.A., The Effects of Pressure and an Acoustic Field on a Cryogenic Coaxial Jet, in 42nd Aerospace Sciences Meeting and Exhibit2004: Reno, NV.

60. Rodriguez, J., Graham, J., Leyva, I., and Talley, D., Effect of Variable Phase Transverse Acoustic Fields on Coaxial Jet Forced Spread Angles, in 47th AIAA Aerospace Sciences Meeting2009: Orlando, FL. 
61. Pomeroy, B., Nugent, N., and Anderson, W., Measuring Transverse Stability Combustion Response at Full Scale Frequencies in a Subscale Combustor, in 46th AIAA/ASME/SAE/ASEE Joint Propulsion Conference2010: Nashville, TN.

62. Marshall, W., Pal, S., Woodward, R., Santoro, R., Smith, R., Xia, G., Sankaran, $\mathrm{V}$., and Merkle, C. Experimental and Computational Investigation of Combustor Acoustics and Instabilities, Part Ii: Transverse Modes. in 44th Aerospace Sciences Meeting and Exhibit. 2006. Reno, NV.

63. Oefelein, J.C. and Yang, V., Modeling High-Pressure Mixing and Combustion Processes in Liquid Rocket Engines. Journal of Propulsion and Power, 1998. 14(5): p. 843-857.

64. Méry, Y., Hakim, L., Scouflaire, P., Vingert, L., Ducruix, S., and Candel, S., Experimental Investigation of Cryogenic Flame Dynamics under Transverse Acoustic Modulations. Comptes Rendus Mécanique, 2013. 341(1): p. 100-109.

65. Sohn, C.H., Park, I.-S., Kim, S.-K., and Jip Kim, H., Acoustic Tuning of GasLiquid Scheme Injectors for Acoustic Damping in a Combustion Chamber of a Liquid Rocket Engine. Journal of sound and vibration, 2007. 304(3): p. 793-810.

66. Sirignano, W.A. and Popov, P.P., Two-Dimensional Model for Liquid-Rocket Transverse Combustion Instability. AIAA Journal, 2013. 51(12): p. 2919-2934.

67. Crocco, L. and Sirignano, W., Effect of the Transverse Velocity Component on the Nonlinear Behavior of Short Nozzles. AIAA Journal, 1966. 4(8): p. 1428-1430.

68. Reardon, F.H., Crocco, L., and Harrje, D.T., Combustion Instability in Liquid Propellant Rocket Motors, 1961, Department of the Navy Bureau of Naval Weapons.

69. Crocco, L., Harrje, D.T., and Reardon, F.H., Transverse Combustion Instability in Liquid Propellant Rocket Motors. ARS Journal, 1962. 32(3): p. 366-373.

70. Hutt, J.J. and Rocker, M., High-Frequency Injection-Coupled Combustion Instability, in Liquid Rocket Engine Combustion Instability, V. Yang and W. Anderson, Editors. 1995, AIAA: Washington D.C. p. 345-356.

71. Hulka, J. and Hutt, J.J., Instability Phenomena in Liquid Oxygen/Hydrogen Propellant Rocket Engines, in Liquid Rocket Engine Combustion Instability, V. Yang and W. Anderson, Editors. 1995, AIAA: Washington D.C. p. 39-72.

72. Vingert, L., Gicquel, P., Lourme, D., and Menoret, L., Coaxial Injector Atomization, in Liquid Rocket Engine Combustion Instability, V. Yang and W. Anderson, Editors. 1995, AIAA: Washington D.C. p. 145-190.

73. Zaller, M.M. and Klem, M.D., Shear Coaxial Injector Spray Characterization, in Liquid Rocket Engine Combustion Instability, V. Yang and W. Anderson, Editors. 1995, AIAA: Washington D.C. p. 191-214.

74. Delplanque, J.-P. and Sirignano, W., Transcritical Liquid Oxygen Droplet Vaporization-Effect on Rocket Combustion Instability. Journal of propulsion and power, 1996. 12(2): p. 349-357.

75. Staffelbach, G., Gicquel, L., Boudier, G., and Poinsot, T., Large Eddy Simulation of Self Excited Azimuthal Modes in Annular Combustors. Proceedings of the Combustion Institute, 2009. 32(2): p. 2909-2916.

76. O'Connor, J. and Acharya, V., Development of a Flame Transfer Function Description for Transversely Forced Flames, in ASME Turbo Expo2013: San Antonio, TX. 
77. Cohen, J. and Proscia, W., Characterization and Control of Aeroengine Combustion Instability: Pratt and Whitney and Nasa Experience, in Combustion Instabilities in Gas Turbine Engines, T.C. Lieuwen and V. Yang, Editors. 2005, AIAA: Washington D.C. p. 113-146.

78. Mongia, H., Held, T., Hsiao, G., and Pandalai, R., Challenges and Progress in Controlling Dynamics in Gas Turbine Combustors. Journal of Propulsion and Power, 2003. 19(5): p. 822-829.

79. Bauerheim, M., Parmentier, J., Salas, P., Nicoud, F., and Poinsot, T., An Analytical Model for Azimuthal Thermoacoustic Modes in an Annular Chamber Fed by an Annular Plenum. Combustion and Flame, 2014. 161: p. 1374-1389.

80. Stow, S., Dowling, A., and Hynes, T., Reflection of Circumferential Modes in a Choked Nozzle. Journal of Fluid Mechanics, 2002. 467(1): p. 215-239.

81. Blackshear, P.L., Rayle, W.D., and Tower, L.K., Study of Screeching Combustion in a 6-Inch Simulated Afterburner. 1955: National Advisory Committee for Aeronautics.

82. Schwing, J., Grimm, F., and Sattelmayer, T., A Model for the Thermo-Acoustic Feedback of Transverse Acoustic Modes and Periodic Oscillations in Flame Position in Cylindrical Flame Tubes, in ASME Turbo Expo2012: Copenhagen, Denmark.

83. Schwing, J. and Sattelmayer, T., High-Frequency Instabilities in Cylindrical Flame Tubes: Feedback Mechanism and Damping, in ASME Turbo Expo2013: San Antonio, TX.

84. Schwing, J., Sattelmayer, T., and Noiray, N., Interaction of Vortex Shedding and Transverse High-Frequency Pressure Oscillations in a Tubular Combustion Chamber, in ASME Turbo Expo2011: Vancouver, Canada.

85. O'Connor, J., Kolb, M., and Lieuwen, T., Visualization of Shear Layer Dynamics in a Transversely Excited, Annular Premixing Nozzle, in 49th AIAA Aerospace Sciences Meeting 2011: Orlando, FL.

86. O'Connor, J. and Lieuwen, T., Disturbance Field Characteristics of a Transversely Excited Burner. Combustion Science and Technology, 2011. 183(5): p. 427-443.

87. O'Connor, J. and Lieuwen, T., Recirculation Zone Dynamics of a Transversely Excited Swirl Flow and Flame. Physics of Fluids, 2012. 24(7): p. 075107-07510730 .

88. O'Connor, J. and Lieuwen, T., Further Characterization of the Disturbance Field in a Transversely Excited Swirl-Stabilized Flame. Journal of Engineering for Gas Turbines and Power - Transactions of the ASME, 2012. 134(1).

89. O'Connor, J. and Lieuwen, T., Influence of Transverse Acoustic Modal Structure on the Forced Response of a Swirling Nozzle Flow, in ASME Turbo Expo2012: Copenhagen, Denmark.

90. O'Connor, J., Mannino, J., C.Vanatta, and Lieuwen, T., Mechanisms for Flame Response in a Transversely Forced Flame, in 7th US National Technical Meeting of the Combustion Institute2011: Atlanta, GA.

91. Malanoski, M., Aguilar, M., Acharya, V., and Lieuwen, T., Dynamics of a Transversely Excited Swirling, Lifted Flame: Part 1 - Experiments and Data Analysis, in ASME Turbo Expo2013: San Antonio, TX. 
92. Malanoski, M., Aguilar, M., O'Connor, J., Shin, D.-h., Noble, B., and Lieuwen, T., Flame Leading Edge and Flow Dynamics in a Swirling, Lifted Flame, in ASME Turbo Expo2012: Copenhagen, Denmark.

93. Acharya, V., Emerson, B., Mondragon, U., Shin, D.-H., Brown, C., McDonell, V., and Lieuwen, T., Velocity and Flame Wrinkling Characteristics of a Transversely Forced, Bluff Body Stabilized Flame, Part Ii: Flame Response Modeling and Comparison with Measurements. Combustion Science and Technology, 2013(just-accepted).

94. Emerson, B., Mondragon, U., Acharya, V., Shin, D.-H., Brown, C., McDonell, V., and Lieuwen, T., Velocity and Flame Wrinkling Characteristics of a Transversely Forced, Bluff Body Stabilized Flame, Part I: Experiments and Data Analysis. Combustion Science and Technology, 2013(just-accepted).

95. Hauser, M., Lorenz, M., and Sattelmayer, T. Influence of Transversal Acoustic Excitation of the Burner Approach Flow on the Flame Structure. in ASME Turbo Expo. 2010. Glasgow, Scotland.

96. Hauser, M., Wagner, M., and Sattelmayer, T., Transformation of Transverse Acoustic Velocity of the Burner Approach Flow into Flame Dynamics, in ASME Turbo Expo2012: Copenhagen, Denmark.

97. Baillot, F. and Lespinasse, F., Response of a Laminar Premixed V-Flame to a High-Frequency Transverse Acoustic Field. Combustion and Flame, 2013.

98. Lespinasse, F., Baillot, F., and Boushaki, T., Responses of V-Flames Placed in an Hf Transverse Acoustic Field from a Velocity to Pressure Antinode. Comptes Rendus Mécanique, 2013. 341(1): p. 110-120.

99. Saurabh, A. and Paschereit, C.O., Combustion Instability in a Swirl Flow Combustor with Transverse Extensions, in ASME Turbo Expo2013: San Antonio, TX.

100. Saurabh, A., Steinert, R., Moeck, J., and Paschereit, C.O., Swirl Flame Response to Traveling Acoustic Waves, in ASME Turbo Expo2014: Dusseldorf, Germany.

101. Worth, N.A. and Dawson, J.R., Self-Excited Circumferential Instabilities in a Model Annular Gas Turbine Combustor: Global Flame Dynamics. Proceedings of the Combustion Institute, 2012.

102. Worth, N.A. and Dawson, J.R., Modal Dynamics of Self-Excited Azimuthal Instabilities in an Annular Combustion Chamber. Combustion and Flame, 2013.

103. Dawson, J.R. and Worth, N.A., The Effect of Baffles on Self-Excited Azimuthal Modes in an Annular Combustor. Proceedings of the Combustion Institute, 2014. in press.

104. Durox, J.-F.B.D., Moeck, J., Schuller, T., and Candel, S., Self-Sustained Instabilities in an Annular Combustor Coupled by Azimuthal and Longitudinal Acoustic Modes, in ASME Turbo Expo2013: San Antonio, TX.

105. Bourgouin, J.-F., Durox, D., Moeck, J., Schuller, T., and Candel, S., Characterization and Modeling of a Spinning Thermoacoustic Instability in an Annular Combustor Equipped with Multiple Matrix Injectors, in ASME Turbo Expo2014: Dusseldorf, Germany.

106. Moeck, J.P. and Paschereit, C.O., Modeling Thermoacoustic Instabilities in an Annular Rijke Tube: Asymmetries and Standing and Spinning Modes. ICSV 16th Proc., Poland, Krakòw, 5-6 July 2009, 2009. 
107. Moeck, J.P., Paul, M., and Paschereit, C.O., Thermoacoustic Instabilities in an Annular Rijke Tube, in ASME Turbo Expo2010, ASME: Glasgow, Scotland.

108. Maslen, S.H. and Moore, F.K., On Strong Transverse Waves without Shocks in a Circular Cylinder. Journal of the Aeronautical Sciences (Institute of the Aeronautical Sciences), 1955. 23(6).

109. Ghirardo, G. and Juniper, M.P., Azimuthal Instabilities in Annular Combustors: Standing and Spinning Modes. Proceedings of the Royal Society A: Mathematical, Physical and Engineering Science, 2013. 469(2157).

110. Burnley, V.S. and CULIC, F.E., On the Energy Transfer between Transverse Acoustic Modes in a Cylindrical Combustion Chamber. Combustion science and technology, 1999. 144(1-6): p. 1-19.

111. Sensiau, C., Nicoud, F., and Poinsot, T., A Tool to Study Azimuthal Standing and Spinning Modes in Annular Combustors. International Journal of Aeroacoustics, 2009. 8(1): p. 57-67.

112. Evesque, S., Polifke, W., and Pankiewitz, C. Spinning and Azimuthally Standing Acoustic Modes in Annular Combustors. in 9th AIAA/CEAS Aeroacoustics Conference. 2003.

113. Polifke, W., Paschereit, C., and Dobbeling, K., Constructive and Destructive Interference of Acoustic and Entropy Waves in a Premixed Combustor with a Choked Exit. International Journal of Acoustics and Vibration, 2001. 6(3): p. 135146.

114. Yang, V. and Culick, F.E.C., On the Existence and Stability of Limit Cycles for Transverse Acoustic Oscillations in a Cylindrical Combustion Chamber. 1: Standing Modes. Combustion Science and Technology, 1990. 72(1-3): p. 37-65.

115. Krebs, W., Walz, G., Flohr, P., and Hoffmann, S., Modal Analysis of Annular Combustors: Effect of the Burner Impedance, in ASME Turbo Expo2001: New Orleans, LA. p. 0042.

116. Pankiewitz, C. and Sattelmayer, T., Time Domain Simulation of Combustion Instabilities in Annular Combustors. Journal of engineering for gas turbines and power, 2003. 125(3): p. 677-685.

117. Nicoud, F., Benoit, L., Sensiau, C., and Poinsot, T., Acoustic Modes in Combustors with Complex Impedances and Multidimensional Active Flames. AIAA Journal, 2007. 45(2): p. 426-441.

118. Campa, G. and Camporeale, S., Prediction of the Thermoacoustic Combustion Instabilities in Practical Annular Combustors. Journal of Engineering for Gas Turbines and Power, 2014. 136(9): p. 091504-1.

119. Camporeale, S., Fortunato, B., and Campa, G., A Finite Element Method for Three-Dimensional Analysis of Thermoacoustic Combustion Instability. Journal of Engineering for Gas Turbines and Power, 2011. 133(1): p. 011506.

120. Noiray, N. and Schuermans, B., On the Dynamic Nature of Azimuthal Thermoacoustic Modes in Annular Gas Turbine Combustion Chambers. Proceedings of the Royal Society A: Mathematical, Physical and Engineering Science, 2013. 469(2151).

121. Noiray, N., Bothien, M., and Schuermans, B., Investigation of Azimuthal Staging Concepts in Annular Gas Turbines. Combustion Theory and Modelling, 2011. 15(5): p. 585-606. 
122. Schuermans, B., Paschereit, C., and Monkewitz, P. Non-Linear Combustion Instabilities in Annular Gas-Turbine Combustors. in 44th AIAA Aerospace Sciences Meeting and Exhibit. 2006. Reno, NV.

123. Parmentier, J.-F., Salas, P., Wolf, P., Staffelbach, G., Nicoud, F., and Poinsot, T., A Simple Analytical Model to Study and Control Azimuthal Instabilities in Annular Combustion Chambers. Combustion and Flame, 2012. 159(7): p. 23742387.

124. You, D., Sun, X., and Yang, V. A Three-Dimensional Linear Acoustic Analysis of Gas Turbine Combustion Instability. in 41 st AIAA Aerospace Sciences Meeting \& Exhibit, Reno, NV. 2003.

125. Dowling, A.P. and Stow, S.R., Acoustic Analysis of Gas Turbine Combustors. Journal of Propulsion and Power, 2003. 19(5): p. 751-764.

126. Graham, O. and Dowling, A., Low-Order Modelling of the Response of Ducted Flames in Annular Geometries, in ASME Turbo Expo2012: Copenhagen, Denmark. p. 479-490.

127. Acharya, V., Shreekrishna, Shin, D.H., and Lieuwen, T., Swirl Effects on Harmonically Excited, Premixed Flame Kinematics. Combustion and Flame, 2012. 159(3): p. 1139-1150.

128. Acharya, V., Malanoski, M., Aguilar, M., and Lieuwen, T., Dynamics of a Transversely Excited Swirling, Lifted Flame: Flame Response Modeling and Comparison with Experiments. Journal of Engineering for Gas Turbines and Power, 2014. 136(5): p. 051503.

129. Acharya, V. and Lieuwen, T. Response of Non-Axisymmetric Premixed, Swirl Flames to Helical Disturbances. in ASME TurboExpo 2014. 2014. Dusseldorf, Germany: ASME.

130. Acharya, V., Shin, D.-H., and Lieuwen, T., Premixed Flames Excited by Helical Disturbances: Flame Wrinkling and Heat Release Oscillations. Journal of Propulsion and Power, 2013. 29(6): p. 1282-1291.

131. Lee, C.Y. and Cant, R.S., Cfd Investigation of Turbulent Premixed Flame Response to Transverse Forcing, in ASME Turbo Expo2013: San Antonio, TX.

132. Wolf, P., Balakrishnan, R., Staffelbach, G., Gicquel, L.Y., and Poinsot, T., Using Les to Study Reacting Flows and Instabilities in Annular Combustion Chambers. Flow, turbulence and combustion, 2012. 88(1-2): p. 191-206.

133. Wolf, P., Staffelbach, G., Roux, A., Gicquel, L., Poinsot, T., and Moureau, V., Massively Parallel Les of Azimuthal Thermo-Acoustic Instabilities in Annular Gas Turbines. Comptes Rendus Mecanique, 2009. 337(6): p. 385-394.

134. Wolf, P., Staffelbach, G., Gicquel, L.Y.M., Muller, J.-D., and Poinsot, T., Acoustic and Large Eddy Simulation Studies of Azimuthal Modes in Annular Combustion Chambers. Combustion and Flame, 2012. 159: p. 3398-3413.

135. Bauerheim, M., Staffelbach, G., Worth, N., Dawson, J., Gicquel, L., and Poinsot, T., Sensitivity of Les-Based Harmonic Flame Response Model for Turbulent Swirled Flames and Impact on the Stability of Azimuthal Modes. Proceedings of the Combustion Institute, 2014.

136. Bauerheim, M., Cazalens, M., and Poinsot, T., A Theoretical Study of Mean Azimuthal Flow and Asymmetry Effects on Thermo-Acoustic Modes in Annular Combustors. Proceedings of the Combustion Institute, 2014. 
137. Fureby, C., Les of a Multi-Burner Annular Gas Turbine Combustor. Flow, turbulence and combustion, 2010. 84(3): p. 543-564.

138. Grinstein, F. and Fureby, C., Les Studies of the Flow in a Swirl Gas Combustor. Proceedings of the combustion institute, 2005. 30(2): p. 1791-1798.

139. Zellhuber, M., Schwing, J., Schuermans, B., Sattelmayer, T., and Polifke, W., Experimental and Numerical Investigation of Thermoacoustic Sources Related to High-Frequency Instabilities. International Journal of Spray and Combustion Dynamics, 2014. 6(1): p. 1-34.

140. Selle, L., Benoit, L., Poinsot, T., Nicoud, F., and Krebs, W., Joint Use of Compressible Large-Eddy Simulation and Helmholtz Solvers for the Analysis of Rotating Modes in an Industrial Swirled Burner. Combustion and Flame, 2006. 145(1): p. 194-205.

141. Martin, C., Benoit, L., Sommerer, Y., Nicoud, F., and Poinsot, T., Large-Eddy Simulation and Acoustic Analysis of a Swirled Staged Turbulent Combustor. AIAA Journal, 2006. 44(4): p. 741-750.

142. Eisenhower, B., Hagen, G., Banaszuk, A., and Mezic, I., Passive Control of Limit Cycle Oscillations in a Thermoacoustic System Using Asymmetry. Journal of Applied Mechanics, 2008. 75(1): p. 011021-011021.

143. Putnam, A.A., Combustion-Driven Oscillations in Industry. 1971: American Elsevier Publishing Company.

144. Richards, G.A., Straub, D.L., and Robey, E.H., Passive Control of Combustion Dynamics in Stationary Gas Turbines. Journal of Propulsion and Power, 2003. 19(5): p. 795-810.

145. Pandalai, R.P. and Mongia, H.C., Combustion Instability Characteristics of Industrial Engine Dry Low Emission Combustion Systems. AIAA Paper, 1998. 3379: p. 1998.

146. Stow, S. and Dowling, A., Modelling of Circumferential Modal Coupling Due to Helmholtz Resonators, in ASME Turbo Expo 20032003: Atlanta, GA.

147. Bellucci, V., Flohr, P., Paschereit, C., and Magni, F., On the Use of Helmholtz Resonators for Damping Acoustic Pulsations in Industrial Gas Turbines. Journal of engineering for gas turbines and power, 2004. 126(2): p. 271-275.

148. Hermann, J., Orthmann, A., Hoffmann, S., and Berenbrink, P., Combination of Active Instability Control and Passive Measures to Prevent Combustion Instabilities in a 260mw Heavy Duty Gas Turbine, 2001, DTIC Document.

149. Gelbert, G., Moeck, J., Bothien, M., King, R., and Paschereit, C. Model Predictive Contorl of Thermoacoustic Instabilities in a Swirl-Stabilized Combustor. in 46th AIAA Aerospace Sciences Meeting and Exhibit. 2008. Reno, NV, USA: AIAA.

150. Illingworth, S.J. and Morgans, A.S., Adaptive Feedback Control of Combustion Instability in Annular Combustors. Combustion Science and Technology, 2010. 182(2): p. 143-164.

151. Hoffmann, S., Weber, G., Judith, H., Hermann, J., and Orthmann, A. Application of Active Combustion Control to Siemens Heavy Duty Gas Turbines. in Symposium of the AVT Panel on Gas Turbine Engine Combustion, Emissions and Alternative Fuels, Lisbon. 1998. 
152. Seume, J.R., Vortmeyer, N., Krause, W., Hermann, J., Hantschk, C.C., Zangl, P., Gleis, S., Vortmayer, D., and Orthmann, A., Application of Active Combustion Instability Control to a Heavy Duty Gas Turbine, 1997.

153. Hermann, J. and Hoffmann, S., Implementation of Active Control in a Full-Scale Gas-Turbine Combustor, in Combustion Instabilities in Gas Turbine Engines, T. Lieuwen and V. Yang, Editors. 2005, AIAA: Washington, D.C.

154. Morgans, A. and Stow, S., Model-Based Control of Combustion Instabilities in Annular Combustors. Combustion and flame, 2007. 150(4): p. 380-399.

155. Fanaca, D., Alemela, P.R., Ettner, F., Hirsch, C., Sattelmayer, T., and Schuermans, B., Determination and Comparison of the Dynamic Characteristics of a Perfectly Premixed Flame in Both Single and Annular Combustion Chambers, in ASME Turbo Expo2008: Berlin, Germany.

156. Kopitz, J., Huber, A., Sattelmayer, T., and Polifke, W. Thermoacoustic Stability Analysis of an Annular Combustion Chamber with Acoustic Low Order Modeling and Validation against Experiment. in ASME Turbo Expo 2005: Power for Land, Sea, and Air. 2005. American Society of Mechanical Engineers.

157. Kunze, K., Hirsch, C., and Sattelmayer, T. Transfer Function Measurements on a Swirl Stabilized Premix Burner in an Annular Combustion Chamber. in ASME Turbo Expo 2004: Power for Land, Sea, and Air. 2004. American Society of Mechanical Engineers.

158. Fanaca, D., Alemela, P., Hirsch, C., and Sattelmayer, T., Comparison of the Flow Field of a Swirl Stabilized Premixed Burner in an Annular and a Single Burner Combustion Chamber. Journal of Engineering for Gas Turbines and Power, 2010. 132(7): p. 071502.

159. Greenblatt, D., Paschereit, C., Lehmann, O., Luchtenburg, D., and Noack, B., Spatiotemporal Characterization of a Conical Swirler Flow Field under Strong Forcing. Journal of Engineering for Gas Turbines and Power, 2009. 131: p. 031504-1.

160. Long, M.R., Bazarov, V.G., and Anderson, W.E., Main Chamber Injectors for Advanced Hydrocarbon Booster Engines, in 39th AIAA/ASME/SAE/ASEE Joint Propulsion Conference2003: Huntsville, AL.

161. Richards, G., Gemmen, R., and Yip, M., A Test Device for Premixed Gas Turbine Combustion Oscillations. Journal of engineering for gas turbines and power, 1997. 119(4): p. 776-782.

162. O'Connor, J., Worth, N.A., and Dawson, J.R., Flame and Flow Dynamics of a Self-Excited, Standing Wave Circumferential Instability in a Model Annular Gas Turbine Combustor, in ASME Turbo Expo2013: San Antonio, TX.

163. Sattelmayer, T. and Polifke, W., Assessment of Methods for the Computation of the Linear Stability of Combustors. Combustion Science and Technology, 2003. 175(3): p. 453-476.

164. Eckstein, J. and Sattelmayer, T., Low-Order Modeling of Low-Frequency Combustion Instabilities in Aeroengines. Journal of propulsion and power, 2006. 22(2): p. 425-432.

165. Sattelmayer, T. and Polifke, W., A Novel Method for the Computaiton of the Linear Stability of Combustors. Combustion Science and Technology, 2003. 175: p. 477-497. 
166. Balasubramanian, K. and Sujith, R., Non-Normality and Nonlinearity in Combustion-Acoustic Interaction in Diffusion Flames. Journal of Fluid Mechanics, 2008. 594: p. 29-57.

167. Balasubramanian, K. and Sujith, R., Nonlinear Response of Diffusion Flames to Uniform Velocity Disturbances. Combustion science and technology, 2008. 180(3): p. 418-436.

168. Yao, Z. and Zhu, M., A Distributed Transfer Function for Non-Premixed Combustion Oscillations. Combustion science and technology, 2012. 184(6): p. 767-790.

169. Markstein, G.H., Nonsteady Flame Propagation. 1964: Pergamon.

170. Williams, F., Turbulent Combustion. the Mathematics of combustion, 1985: p. 97131.

171. Fleifil, M., Annaswamy, A.M., Ghoneim, Z.A., and Ghoniem, A.F., Response of a Laminar Premixed Flame to Flow Oscillations: A Kinematic Model and Thermoacoustic Instability Results. Combustion and Flame, 1996. 106(4): p. 487510.

172. Dowling, A., A Kinematic Model of a Ducted Flame. Journal of Fluid Mechanics, 1999. 394: p. 51-72.

173. Schuller, T., Durox, D., and Candel, S., A Unified Model for the Prediction of Laminar Flame Transfer Functions: Comparisons between Conical and V-Flame Dynamics. Combustion and Flame, 2003. 134(1): p. 21-34.

174. Kim, D., Lee, J.G., Quay, B.D., Santavicca, D.A., Kim, K., and Srinivasan, S., Effect of Flame Structure on the Flame Transfer Function in a Premixed Gas Turbine Combustor. Journal of Engineering for Gas Turbines \& Power, 2010. 22(2): p. 021502.

175. Alemela, P.R., Fanaca, D., Ettner, F., Hirsch, C., Sattelmayer, T., and Schuermans, B. Flame Transfer Matrices of a Premixed Flame and a Global Check with Modelling and Experiments. 2008. ASME.

176. Preetham, Santosh, H., and Lieuwen, T., Dynamics of Laminar Premixed Flames Forced by Harmonic Velocity Disturbances. Journal of Propulsion and Power, 2008. 24(6): p. 1390-1402.

177. Noiray, N., Durox, D., Schuller, T., and Candel, S., A Unified Framework for Nonlinear Combustion Instability Analysis Based on the Flame Describing Function. Journal of Fluid Mechanics, 2008. 615(1): p. 139-167.

178. Bellows, B.D., Neumeier, Y., and Lieuwen, T., Forced Response of a Swirling, Premixed Flame to Flow Disturbances. Journal of propulsion and power, 2006. 22(5): p. 1075-1084.

179. Acharya, V., Malanoski, M., Aguilar, M., and Lieuwen, T., Dynamics of a Transversely Excited Swirling, Lifted Flame, Part Ii: Flame Response Modeling and Comparison with Experiments, in ASME Turbo Expo2013: San Antonio, TX.

180. Magina, N. and Lieuwen, T., Response of over-Ventilated Non-Premixed Flames to Transverse Flow Perturbations, in Fall Technical Meeting of the Eastern States Section of the Combustion Institute2013: Clemson, SC.

181. Magina, N., Acharya, V., and Lieuwen, T., Response of Non-Premixed Flames to Bulk Flow Perturbations. Proceedings of the Combustion Institute, 2013. 34(1): p. 963-971. 
182. Merk, H., Analysis of Heat-Driven Oscillations of Gas Flows: General Considerations. Applied Scientific Research, Section A, 1957. 6(4): p. 317-336.

183. Putnam, A., Combustion-Driven Oscillations in Industry. 1971, New York: Elsevier.

184. Bloxsidge, G., Dowling, A., and Langhorne, P., Reheat Buzz: An Acoustically Coupled Combustion Instability, Part 2. Theory. Journal of Fluid Mechanics, 1988. 193: p. 445-473.

185. Heckl, M., Active Control of the Noise from a Rijke Tube, in Aero-and HydroAcoustics. 1986, Springer Berlin Heidelberg. p. 211-216.

186. Bohn, D. and Deuker, E., An Acoustical Model to Predict Combustion Driven Oscillations, in 20th International Congress on Combustion Engines1993: London, UK.

187. Dowling, A., The Calculation of Thermoacoustic Oscillations. Journal of sound and vibration, 1995. 180(4): p. 557-581.

188. Dowling, A.P. and Morgans, A.S., Feedback Control of Combustion Oscillations. Annual Review of Fluid Mechanics, 2005. 37(1): p. 151-182.

189. Munjal, M., Acoustics of Ducts and Mufflers. 1986: John Wiley \& Sons.

190. Crocco, L. and Mitchell, C., Nonlinear Periodic Oscillations in Rocket Motors with Distributed Combustion. Combustion science and technology, 1969. 1(2): p. 146-169.

191. Culick, F., Some Recent Results for Nonlinear Acoustics in Combustion Chambers. AIAA Journal, 1994. 32(1): p. 146-169.

192. Culick, F. and Yang, V., Overview of Combustion Instabilities in LiquidPropellant Rocket Engines, in Liquid Rocket Engine Combustion Instability, V. Yang and W. Anderson, Editors. 1995, AIAA: Washington D.C. p. 3-38.

193. Stow, S., Dowling, A., and Hynes, T., Reflection of Circumferential Modes in a Choked Nozzle. Journal of Fluid Mechanics, 2002. 467: p. 215-239.

194. Evesque, S.p. and Polifke, W. Low-Order Acoustic Modelling for Annular Combustors: Validation and Inclusion of Modal Coupling. in ASME Turbo Expo 2002: Power for Land, Sea, and Air. 2002. American Society of Mechanical Engineers.

195. Blimbaum, J., Zanchetta, M., Akin, T., Acharya, V., O'Connor, J., Noble, D., and Lieuwen, T., Transverse to Longitudinal Acoustic Coupling Processes in Annular Combustion Chambers. International Journal of Spray and Combustion Dynamics, 2012. 4(4): p. 275-298.

196. Stow, S.R. and Dowling, A.P. Low-Order Modelling of Thermoacoustic Limit Cycles. 2004.

197. Worth, N.A. and Dawson, J.R., Cinematographic Oh-Plif Measurements of Two Interacting Turbulent Premixed Flames with and without Acoustic Forcing. Combustion and Flame, 2011.

198. Pierce, A.D., Acoustics: An Introduction to Its Physical Principles and Applications. 1989: Acoustical Society of America.

199. Staffelbach, G., Gicquel, L.Y.M., Boudier, G., and Poinsot, T., Large Eddy Simulation of Self Excited Azimuthal Modes in Annular Combustors. Proceedings of the Combustion Institute, 2009. 32: p. 2909-2916. 
200. Tsien, H.S., The Transfer Function of Rocket Nozzles. Journal of the American Rocket Society, 1952. 22: p. 139-143.

201. Crocco, L., Supercritical Gaseous Discharge with High Frequency Oscillations. Aerotechnica, 1953. 33: p. 46-53.

202. Crocco, L. and Sirignano, W.A., Behavior of Supercritical Nozzles under ThreeDimensional Oscillatory Conditions, in AGARDograph1967, AGARD.

203. Crocco, L. and Sirignano, W.A., Effect of Transverse Velocity Component on the Nonlinear Behavior of Short Nozzles. AIAA Journal, 1966. 4(8): p. 1428-1430.

204. Marble, F., Acoustic Disturbance from Gas Non-Uniformities Convecting through a Nozzle, in Symposium on Transportation Noise 1973: Stanford, CA, USA.

205. Marble, F. and Candel, S., Acoustic Disturbance from Gas Non-Uniformities Convected through a Nozzle. Journal of Sound and Vibration, 1977. 55(2): p. 225243.

206. Levine, H. and Schwinger, J., On the Radiation of Sound from an Unflanged Circular Pipe. Physical Review, 1948. 73(4): p. 383-406.

207. Brown, G.L. and Roshko, A., On Density Effects and Large Structure in Turbulent Mixing Layers. Journal of Fluid Mechanics, 1974. 64(4): p. 775-816.

208. Barkley, D., Linear Analysis of the Cylinder Wake Mean Flow. Europhysics Letters, 2006. 75(5): p. 750.

209. Meliga, P., Pujals, G., and Serre, E., Sensitivity of 2-D Turbulent Flow Past a DShaped Cylinder Using Global Stability. Physics of Fluids, 2012. 24(6): p. 061701.

210. Mettot, C., Sipp, D., and Bezard, H., Quasi-Laminar Stability and Sensitivity Analyses for Turbulent Flows: Prediction of Low-Frequency Unsteadiness and Passive Control. Physics of Fluids, 2014. 26(4): p. 045112.

211. Yu, M.-H. and Monkewitz, P., The Effect of Nonuniform Density on the Absolute Instability of Two-Dimensional Inertial Jets and Wakes. Physics of Fluids A: Fluid Dynamics, 1990. 2(7): p. 1175-1181.

212. Schmid, P. and Henningson, D., Stability and Transition in Shear Flows. Applied Mathematical Sciences. Vol. 142. 2001: Springer.

213. Yildirim, B. and Agrawal, A., Full-Field Measurements of Self-Excited Oscillations in Momentum-Dominated Helium Jets. Experiments in Fluids, 2005. 38(2): p. 161-173.

214. Morris, P., The Spatial Viscous Instability of Axisymmetric Jets. Journal of Fluid Mechanics, 1976. 77(03): p. 511-529.

215. Batchelor, G. and Gill, A., Analysis of the Stability of Axisymmetric Jets. Journal of Fluid Mechanics, 1962. 14(04): p. 529-551.

216. Pier, B., Local and Global Instabilities in the Wake of a Sphere. Journal of Fluid Mechanics, 2008. 603: p. 39-61.

217. Monkewitz, P., A Note on Vortex Shedding from Axisymmetric Bluff Bodies. Journal of Fluid Mechanics, 1988. 192: p. 561-575.

218. Rusak, Z., Kapila, A., and Choi, J.J., Effect of Combustion on near-Critical Swirling Flow. Combustion Theory and Modelling, 2002. 6(4): p. 625-645.

219. Emerson, B., O'Connor, J., Juniper, M., and Lieuwen, T., Density ratio effects on reacting bluffbody flow field characteristics. Journal of Fluid Mechanics, 2012. 706: p. 219-250. 
220. Emerson, B., O'Connor, J., Noble, D., and Lieuwen, T., Frequency Locking and Vortex Dynamics of an Acoustically Excited Bluff Body Stabilized Flame, in 50th AIAA Aerospace Sciences Meeting, 2012: Nashville, TN.

221. Syred, N., A Review of Oscillation Mechanisms and the Role of the Precessing Vortex Core $(P v c)$ in Swirl Combustion Systems. Progress in Energy and Combustion Science, 2006. 32(2): p. 93-161.

222. Billant, P., Chomaz, J., and Huerre, P., Experimental Study of Vortex Breakdown in Swirling Jets. Journal of Fluid Mechanics, 1998. 376: p. 183-219.

223. Fick, W., Griffiths, A., and O'Doherty, T., Visualisation of the Precessing Vortex Core in an Unconfined Swirling Flow. Optical Diagnostics in Engineering, 1997. 2(1): p. 19-31.

224. Ho, C.-M. and Huang, L.-S., Subharmonics and Vortex Merging in Mixing Layers. Journal of Fluid Mechanics, 1982. 119: p. 443-473.

225. Roberts, F. and Roshko, A., Effects of Periodic Forcing on Mixing in Turbulent Shear Layers and Wakes, 1985, DTIC Document.

226. Oster, D. and Wygnanski, I., The Forced Mixing Layer between Parallel Streams. Journal of Fluid Mechanics, 1982. 123: p. 91-130.

227. Greenblatt, D. and Wygnanski, I.J., The Control of Flow Separation by Periodic Excitation. Progress in Aerospace Sciences, 2000. 36(7): p. 487-545.

228. Bechert, D. and Stahl, B., Excitation of Instability Waves in Free Shear Layers Part 2. Experiments. Journal of Fluid Mechanics, 1988. 186: p. 63-84.

229. Chun, K.-B. and Sung, H., Control of Turbulent Separated Flow over a Backward-Facing Step by Local Forcing. Experiments in Fluids, 1996. 21(6): p. 417-426.

230. Chun, S., Lee, I., and Sung, H.J., Effect of Spanwise-Varying Local Forcing on Turbulent Separated Flow over a Backward-Facing Step. Experiments in Fluids, 1999. 26(5): p. 437-440.

231. Hasan, M., The Flow over a Backward-Facing Step under Controlled Perturbation: Laminar Separation. Journal of Fluid Mechanics, 1992. 238: p. $73-$ 96.

232. Marrot, F., Gajan, P., Pauzin, S., and Simon, F., Experimental Application of an Active Control Loop on Backward-Facing Step Flow. AIAA journal, 2005. 43(6): p. 1176-1186.

233. Fiedler, H. and Mensing, P., The Plane Turbulent Shear Layer with Periodic Excitation. Journal of Fluid Mechanics, 1985. 150(1): p. 281-309.

234. Tam, C.K., Excitation of Instability Waves in a Two-Dimensional Shear Layer by Sound. Journal of Fluid Mechanics, 1978. 89(02): p. 357-371.

235. Kiya, M., Shimizu, M., and Mochizuki, O., Sinusoidal Forcing of a Turbulent Separation Bubble. Journal of Fluid Mechanics, 1997. 342: p. 119-139.

236. Sigurdson, L., The Structure and Control of a Turbulent Reattaching Flow. Journal of Fluid Mechanics, 1995. 298: p. 139-165.

237. Mills, R., Sheridan, J., and Hourigan, K., Response of Base Suction and Vortex Shedding from Rectangular Prisms to Transverse Forcing. Journal of Fluid Mechanics, 2002. 461: p. 25-49.

238. Nishioka, M., Asai, M., and Yoshida, S., Control of Flow Separation by Acoustic Excitation. AIAA journal, 1990. 28(11): p. 1909-1915. 
239. Yang, S. and Spedding, G., Separation Control by External Acoustic Excitation at Low Reynolds Numbers. AIAA journal, 2013. 51(6): p. 1506-1515.

240. Yarusevych, S., Sullivan, P.E., and Kawall, J.G., Effect of Acoustic Excitation Amplitude on Airfoil Boundary Layer and Wake Development. AIAA journal, 2007. 45(4): p. 760-771.

241. Zaman, K., Bar-Sever, A., and Mangalam, S., Effect of Acoustic Excitation on the Flow over a Low-Re Airfoil. Journal of Fluid Mechanics, 1987. 182: p. 127-148.

242. Zaman, K.B., Effect of Acoustic Excitation on Stalled Flows over an Airfoil. AIAA journal, 1992. 30(6): p. 1492-1499.

243. Raman, G., Rice, E., and Reshotko, E., Mode Spectra of Natural Disturbances in a Circular Jet and the Effect of Acoustic Forcing. Experiments in fluids, 1994. 17(6): p. 415-426.

244. Arbey, H. and Williams, J., Active Cancellation of Pure Tones in an Excited Jet. Journal of Fluid Mechanics, 1984. 149: p. 445-454.

245. Bechert, D. and Pfizenmaier, E., On the Amplification of Broad Band Jet Noise by a Pure Tone Excitation. Journal of Sound and Vibration, 1975. 43(3): p. 581-587.

246. Hussain, A. and Hasan, M., Turbulence Suppression in Free Turbulent Shear Flows under Controlled Excitation. Part 2. Jet-Noise Reduction. Journal of Fluid Mechanics, 1985. 150: p. 159-168.

247. Kibens, V., Discrete Noise Spectrum Generated by Acoustically Excited Jet. AIAA Journal, 1980. 18(4): p. 434-441.

248. Parekh, D., Kibens, V., Glezer, A., Wiltse, J., and Smith, D., Innovative Jet Flow Control: Mixing Enhancement Experiments. AIAA paper, 1996. 308: p. 1996.

249. Corke, T. and Kusek, S., Resonance in Axisymmetric Jets with Controlled Helical-Mode Input. Journal of Fluid Mechanics, 1993. 249: p. 307-336.

250. Kusek, S., Corke, T., and Reisenthel, P., Seeding of Helical Modes in the Initial Region of an Axisymmetric Jet. Experiments in Fluids, 1990. 10(2-3): p. 116-124.

251. Zaman, K. and Hussain, A., Turbulence Suppression in Free Shear Flows by Controlled Excitation. Journal of Fluid Mechanics, 1981. 103: p. 133-159.

252. Suzuki, H., Kasagi, N., and Suzuki, Y., Active Control of an Axisymmetric Jet with Distributed Electromagnetic Flap Actuators. Experiments in fluids, 2004. 36(3): p. 498-509.

253. Cattafesta III, L.N. and Sheplak, M., Actuators for Active Flow Control. Annual Review of Fluid Mechanics, 2011. 43: p. 247-272.

254. Matta, L., Wuersig, H., Choung, A., Jagoda, J., and Zinn, B. Excitation of a Round Jet by Transverse Standing Acoustic Oscillations. in 34th Aerospace Sciences Meeting and Exhibit. 1996.

255. Suzuki, M., Atarashi, T., and Masuda, W., Behavior and Structure of Internal Fuel-Jet in Diffusion Flame under Transverse Acoustic Excitation. Combustion Science and Technology, 2007. 179(12): p. 2581-2597.

256. Morkovin, M. and Paranjape, S., On Acoustic Excitation of Shear Layers. Zeitschrift für Flugwissenschaften, 1971. 19: p. 328-335.

257. Kozlov, V., Grek, G., Litvinenko, Y.A., Kozlov, G., and Litvinenko, M., Round and Plane Jets in a Transverse Acoustic Field. Journal of Engineering Thermophysics, 2011. 20(3): p. 272-289. 
258. Reynolds, W., Parekh, D., Juvet, P., and Lee, M., Bifurcating and Blooming Jets. Annual review of fluid mechanics, 2003. 35(1): p. 295-315.

259. Gursul, I., Effect of Nonaxisymmetric Forcing on a Swirling Jet with Vortex Breakdown. Journal of fluids engineering, 1996. 118(2): p. 316-321.

260. Gallaire, F., Rott, S., and Chomaz, J.-M., Experimental Study of a Free and Forced Swirling Jet. Physics of Fluids (1994-present), 2004. 16(8): p. 2907-2917.

261. Panda, J. and McLaughlin, D., Experiments on the Instabilities of a Swirling Jet. Physics of Fluids (1994-present), 1994. 6(1): p. 263-276.

262. Baillot, F., Blaisot, J.-B., Boisdron, G., and Dumouchel, C., Behaviour of an AirAssisted Jet Submitted to a Transverse High-Frequency Acoustic Field. Journal of Fluid Mechanics, 2009. 640: p. 305-342.

263. Carpentier, J.-B., Baillot, F., Blaisot, J.-B., and Dumouchel, C., Behavior of Cylindrical Liquid Jets Evolving in a Transverse Acoustic Field. Physics of Fluids (1994-present), 2009. 21(2): p. 023601.

264. Greenberg, J. and Katoshevski, D., Vaporization Damköhler Number and Enrichment Effects in Spray Diffusion Flames in an Oscillating Flow Field. Combustion Science and Technology, 2013. 185(6): p. 898-919.

265. Leyva, I.A., Chehroudi, B., and Talley, D., Dark Core Analysis of Coaxial Injectors at Sub-, near-, and Supercritical Pressures in a Transverse Acoustic Field. AIAA, 2007. 5456: p. 2007.

266. Richecoeur, F., Scouflaire, P., Ducruix, S., and Candel, S., High-Frequency Transverse Acoustic Coupling in a Multiple-Injector Cryogenic Combustor. Journal of Propulsion and Power, 2006. 22(4): p. 790-799.

267. Hardi, J.S., Martinez, H.C.G., Oschwald, M., and Dally, B.B., Lox Jet Atomization under Transverse Acoustic Oscillations. Journal of Propulsion and Power, 2014. 30(2): p. 337-349.

268. Liu, T., Zong, N., and Yang, V. Dynamics of Shear-Coaxial Cryogenic Nitrogen Jets with Acoustic Excitation under Supercritical Conditions. in 44th AIAA Aerospace Sciences Meeting and Exhibit. 2006.

269. Sevilla-Esparza, C.I., Wegener, J.L., Teshome, S., Rodriguez, J.I., Smith, O.I., and Karagozian, A.R., Droplet Combustion in the Presence of Acoustic Excitation. Combustion and Flame, 2014. 161(6): p. 1604-1619.

270. Larsen, P.S. and Jensen, J.W., Evaporation Rates of Drops in Forced Convection with Superposed Transverse Sound Field. International Journal of Heat and Mass Transfer, 1978. 21(4): p. 511-517.

271. Leyva, I.A., Rodriguez, J.I., Chehroudi, B., and Talley, D. Preliminary Results on Coaxial Jet Spread Angles and the Effects of Variable Phase Transverse Acoustic Fields. in 46th AIAA Aerospace Sciences Meeting and Exhibit, Reno, Nevada, Jan. 2008.

272. Eckstein, J., Freitag, E., Hirsch, C., Sattelmayer, T., von der Bank, R., and Schilling, T. Forced Low-Frequency Spray Characteristics of a Generic Airblast Swirl Diffusion Burner. in ASME Turbo Expo 2003: Power for Land, Sea and Air. 2003. Atlanta, GA, USA: ASME.

273. Kim, W., Zhang, S., Palies, P., Cohen, J., Liljenberg, S., and Hautman, D. The Behavior of Liquid Fuel Sprays in Acoustically-Forced Air Swirler Flows. in ASME Turbo Expo 2012. 2012. Copenhagen, Denmark: ASME. 
274. Zhu, M., Dowling, A., and Bray, K., Forced Oscillations in Combustors with Spray Atomizers. Journal of Engineering for Gas Turbines and Power, 2002. 124(1): p. 20-30.

275. Cho, J. and Lieuwen, T., Laminar Premixed Flame Response to Equivalence Ratio Oscillations. Combustion and Flame, 2005. 140(1): p. 116-129.

276. Polifke, W. and Lawn, C., On the Low-Frequency Limit of Flame Transfer Functions. Combustion and Flame, 2007. 151(3): p. 437-451.

277. Boyer, L. and Quinard, J., On the Dynamics of Anchored Flames. Combustion and Flame, 1990. 82: p. 51-65.

278. Moeck, J.P., Bourgouin, J.-F., Durox, D., Schuller, T., and Candel, S., Nonlinear Interaction between a Precessing Vortex Core and Acoustic Oscillations in a Turbulent Swirling Flame. Combustion and Flame, 2012. 159(8): p. 2650-2668.

279. Stohr, M., Boxx, I., Carter, C.D., and Meier, W., Experimental Study of VortexFlame Interaction in a Gas Turbine Model Combustor. Combustion and Flame, 2012. 159(8): p. 2636-2469.

280. Lighthill, J., Waves in Fluids. 2001: Cambridge University Press.

281. Crighton, D., The Kutta Condition in Unsteady Flow. Annual review of fluid mechanics, 1985. 17(1): p. 411-445.

282. Crawford, J., Factors That Limit Control Effectiveness in Self-Excited Noise Driven Combustors, in Aerospace Engineering2012, Georgia Institute of Technology: Atlanta, GA, USA.

283. Li, L. and Sun, X., Effect of Vorticity Waves on Azimuthal Instabilities in Annular Chambers. Combustion and Flame, 2014. 


\section{Figure Captions}

Figure 1 - Thermoacoustic feedback cycle.

Figure 2 - Conceptual model of an annular combustor undergoing azimuthal combustion instability.

Figure 3 - Model annular combustor configurations from (a) Worth and Dawson [102] and (b) Bourgouin et al. [104].

Figure 4 - Conceptual drawing of transverse velocity coupled combustion instability (a), and spark-schlieren photograph of the resulting oscillations (b), reproduced from Rogers and Marble [9].

Figure 5 - Quartz cylindrical combustor at atmospheric conditions for high-frequency, self-excited oscillation studies from Schwing et al. [84].

Figure 6 - Conceptual view of the General Electric tunable combustor acoustic (TCA) test rig with a perforated piston to vary plenum length (A), single injector (B), combustion chamber with flame (C), and perforated piston to vary combustor length (D), adapted from Mongia et al. [78].

Figure 7 - Combustor design concept for transversely forced experiments, Adapted from Malanoski et al. [91].

Figure 8 - Transverse forcing facilities in the unwrapped annular combustor configuration with high aspect ratio combustors and speaker tubes: (a) Experiment of O'Connor and Lieuwen [87], and (b) Experiment of Malanoski et al. [92].

Figure 9 - (a) Acoustic forcing configuration and (b) acoustic field measurements for the transverse forcing facility of Lespinasse $e t$ al. [98]. Filled symbols are acoustic pressure measurements and open symbols are acoustic velocity measurements.

Figure 10 - T-shape transverse forcing experiments from (a) Hauser et al. [95] and (b) Saurabh and Paschereit [99], capable of both transverse and longitudinal forcing.

Figure 11 - Extent of flame interaction in an annular combustor measured via timeaverage $\mathrm{OH}^{*}$ chemiluminescence from an overhead view. Images show two nozzle spacings - (a) 2.33 and (b) 1.56 times the nozzle diameter - where all swirlers have a counterclockwise rotation direction. Reproduced from Worth and Dawson [102].

Figure 12 - Pressure (left) and temperature (right) fields from LES simulation of a fullannular combustor during azimuthal acoustic mode oscillations. Reproduced from Wolf et al. [134]. 
Figure 13 - General Electric LM6000 DLE combustor cross-section with damper tube in combustor plenum and circumferential baffles. Reproduced from Mongia et al. [78].

Figure 14 - Dependence of (a) azimuthal and (b) radial natural frequencies of annular geometry upon normalized gap width; following Lieuwen [195].

Figure 15 - Joint probability of forward and backward spinning wave amplitudes for an $m_{a}=1$ azimuthal instability in a model annular combustor with a) counterclockwise swirling nozzles and b) alternating swirl between clockwise and counterclockwise, and c) two baffles placed at $180^{\circ}$ angles around the annulus. Adapted from Worth and Dawson $[102,103]$.

Figure 16 - Joint probability density functions of standing wave amplitudes in an azimuthally unstable annular combustor at three noise levels. Adapted from Noiray et al. [120].

Figure 17 - Standing acoustic pressure and velocity fields shown relative to inflow nozzle. The $x$-coordinate corresponds to the azimuthal coordinate $\theta$ in the full annular combustor, where $x=R \theta$.

Figure 18 - Schematic of transverse pressure fluctuations as experienced by nozzle for pressure anti-node (a) and pressure node (b) forcing cases.

Figure 19 - Pressure amplitudes at the center plane $(y=0)$ for $D / \lambda \approx 0.04(400 \mathrm{~Hz})$ with (a) an anechoic nozzle for the pressure node case, and (b) pressure release condition at the nozzle for the pressure anti-node case. Reproduced from Blimbaum et al. [195].

Figure 20 - Experimental results of PIV measurements of velocity (vectors) and normalized vorticity (color) fluctuation for $400 \mathrm{~Hz}$ asymmetric (a) and $400 \mathrm{~Hz}$ symmetric (b) forcing in non-reacting swirling flow at $u_{z, 0}=10 \mathrm{~m} / \mathrm{s}, S=0.85$. Experimental flame luminescence images of $400 \mathrm{~Hz}$ asymmetric (c) and $400 \mathrm{~Hz}$ symmetric (d) forcing of a flame in a swirling flow at $u_{z, 0}=10 \mathrm{~m} / \mathrm{s}, S=0.5$, and an equivalence ratio of 0.9 . Arrows point to the wrinkles resulting from c) helical and d) ring vortices.

Figure 21 - Examples of symmetric and asymmetric flame oscillations in an annular combustor. Flames displaying (a) symmetric (ring vortex) and (b) asymmetric (helical vortex) response are shown using the fluctuation amplitude of $\mathrm{OH}^{*}$ chemiluminescence imaging from an overhead view [103]. In (c) and (d), OH-PLIF images of flames at pressure anti-nodes and velocity anti-nodes, respectively, with arrows showing similar flame wrinkling to Figure 20 [162].

Figure 22 - Simplified representation of Figure 2 for a model thin annular combustor with a temperature discontinuity interface. 
Figure 23 - Effect of azimuthal modes $(m)$ on the normalized natural frequency in a thin annular duct with $L / R=0.5$ and $\kappa=T_{b, 0} / T_{u, 0}$. "PR" denotes a pressure release outflow boundary condition.

Figure 24 - Representation of modes for $m_{h}=0$ (ring vortices), $m_{h}>0$ (clockwisewinding helix), and $m_{h}<0$ (counter-clockwise-winding helix).

Figure 25 - Linear spatial growth rate of hydrodynamic modes in a swirling jet as a function of excitation frequency. Plot courtesy of Benjamin Emerson.

Figure 26 - Top-view of nozzle showing Cartesian and cylindrical polar coordinate systems with respect to the center of the nozzle.

Figure 27 - Amplitude of helical modes excited by (a) symmetric forcing and (b) asymmetric acoustic forcing. Illustrative results from transversely forced cold-flow experiment by O'Connor and Lieuwen [87] showing modal amplitudes for $1200 \mathrm{~Hz}$ (c) symmetric forcing and (d) asymmetric forcing.

Figure 28 - Response of a circular jet to (a) symmetric and (b) asymmetric (forcing of the $m_{h}= \pm 1$ modes) acoustic forcing around the circumference of the jet. Images reproduced from Kusek et al. [250].

Figure 29. Response of a liquid coaxial jet in a transverse acoustic at a range of phases between the speakers at either end of a transverse forcing facility [271].

Figure 30 - Flame coupling pathways in transversely excited flames.

Figure 31 - Time-delays between the unsteady pressure and intermediate processes affecting the heat release: (a) Effect of transverse velocity oscillations, (b) Effect of fuel/air ratio oscillations from induced longitudinal velocity oscillations, (c) Effect of flow instabilities.

Figure 32 - Velocity fluctuations during (a) longitudinal forcing; (b) transverse forcing.

Figure 33 - Flame transfer function $(F)$ amplitude comparison between symmetric (black) and asymmetric (gray) forcing. Data reproduced from Acharya et al. [128].

Figure 34 - Cut-section side view along the circumferential direction, showing the control volume and the coordinate system.

Figure 35 - Variation of instability growth rate for the 1 case for the fundamental frequency $m_{a}=1$ and the second mode $m_{a}=2$, with the time-delay parameter $\tilde{\tau}_{q}$ for $n=1.5$. 
Figures

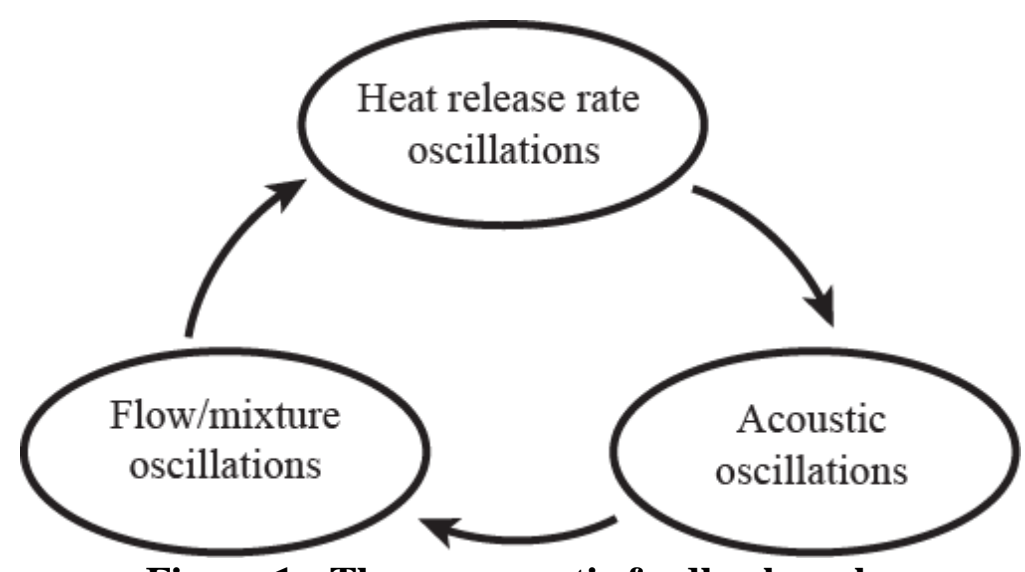

Figure 1 - Thermoacoustic feedback cycle. 


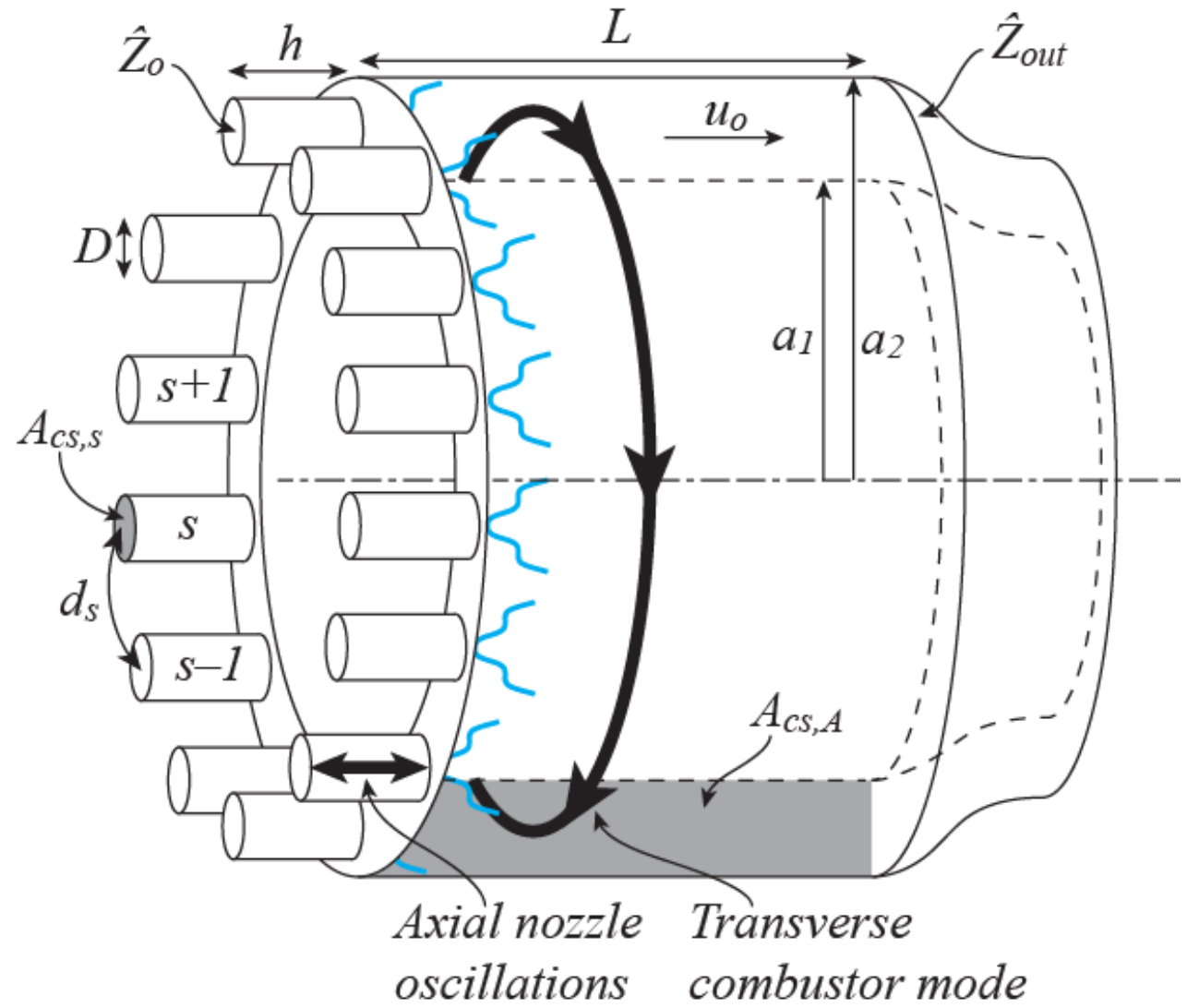

Figure 2 - Conceptual model of an annular combustor undergoing azimuthal combustion instability. 


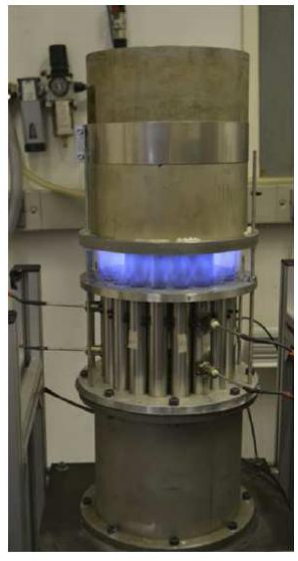

(a)

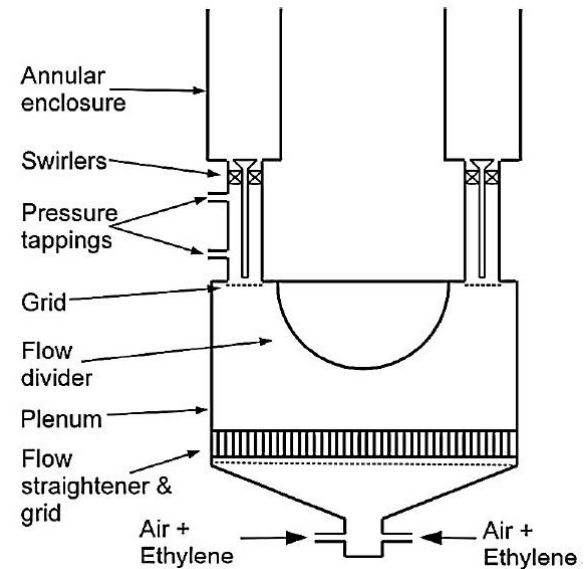

Ethylene Ethylene

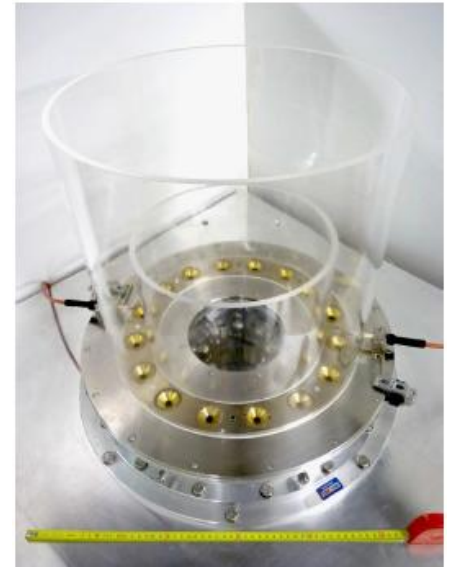

(b)

Figure 3 - Model annular combustor configurations from (a) Worth and Dawson [102] and (b) Bourgouin et al. [104]. 


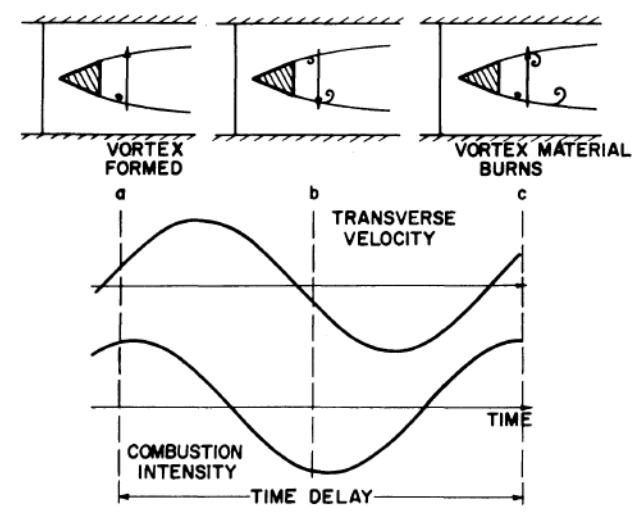

(a)

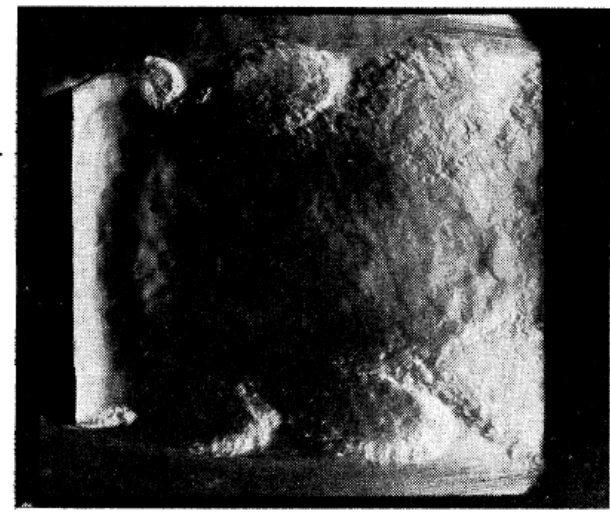

(b)

Figure 4 - Conceptual drawing of transverse velocity coupled combustion instability (a), and spark-schlieren photograph of the resulting oscillations (b), reproduced from Rogers and Marble [9]. 


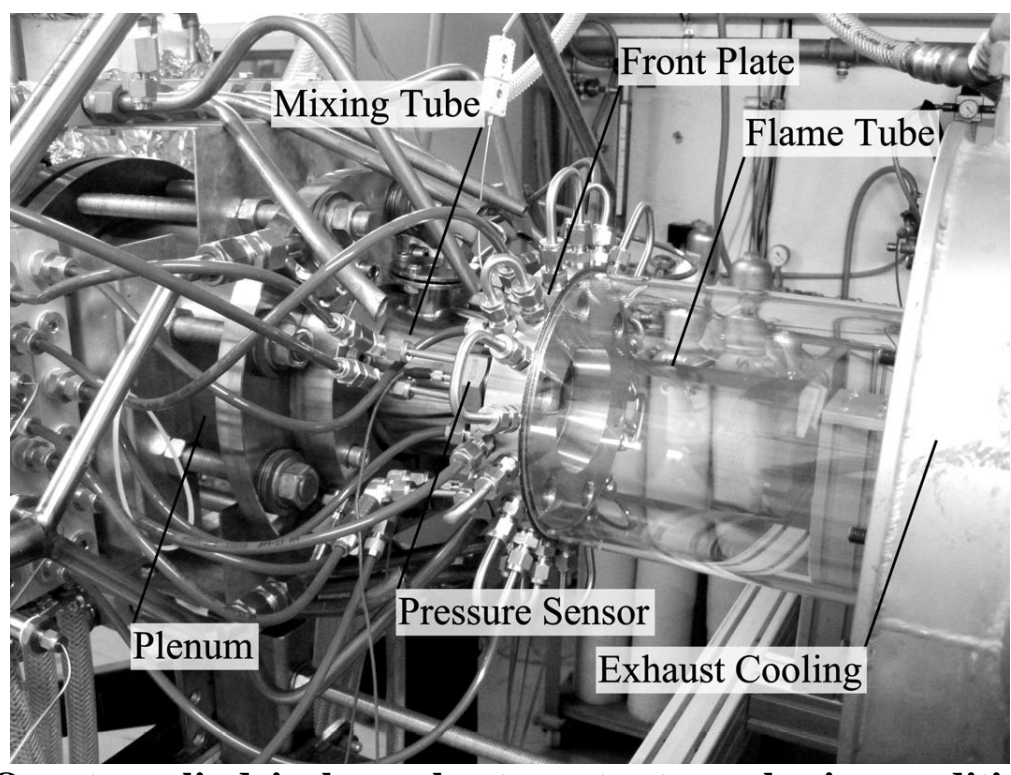

Figure 5 - Quartz cylindrical combustor at atmospheric conditions for highfrequency, self-excited oscillation studies from Schwing et al. [84]. 


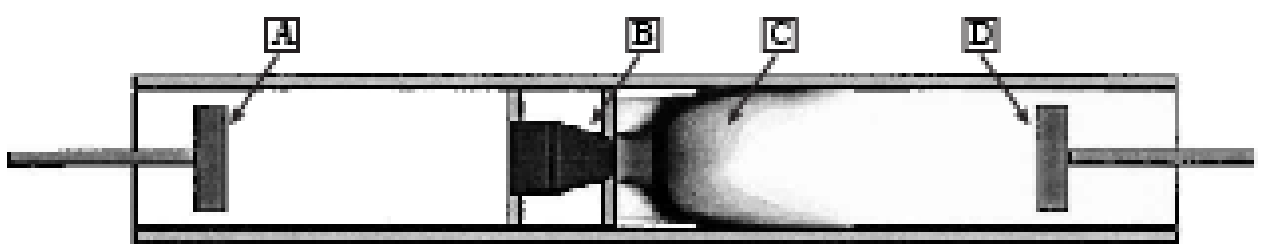

Figure 6 - Conceptual view of the General Electric tunable combustor acoustic (TCA) test rig with a perforated piston to vary plenum length (A), single injector (B), combustion chamber with flame $(C)$, and perforated piston to vary combustor length (D), adapted from Mongia et al. [78]. 


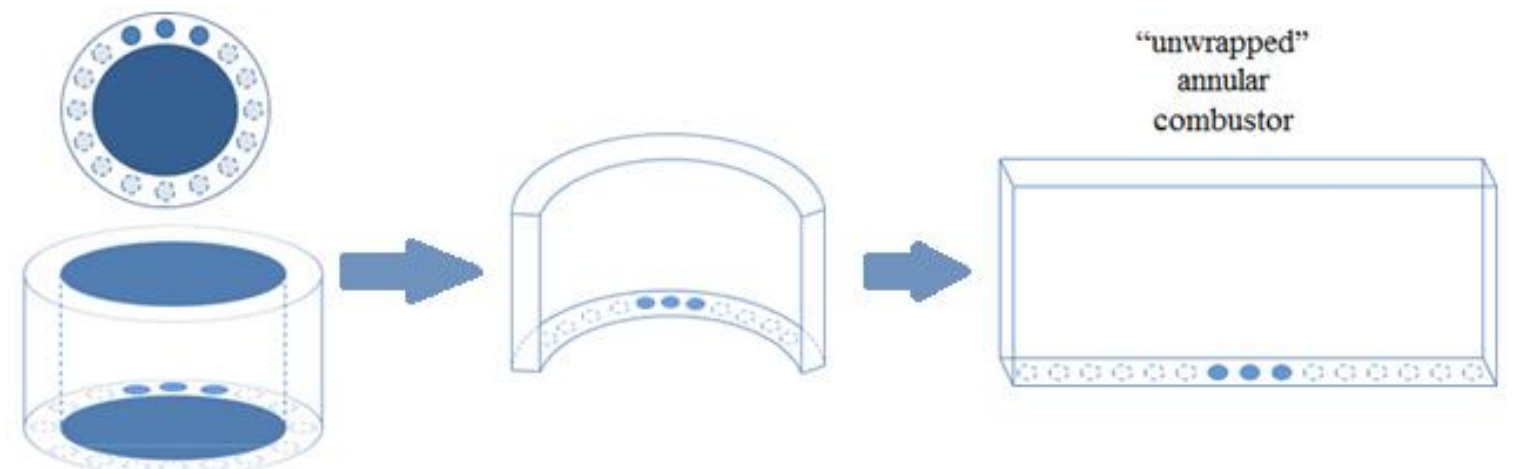

Figure 7 - Combustor design concept for transversely forced experiments, Adapted from Malanoski et al. [91]. 


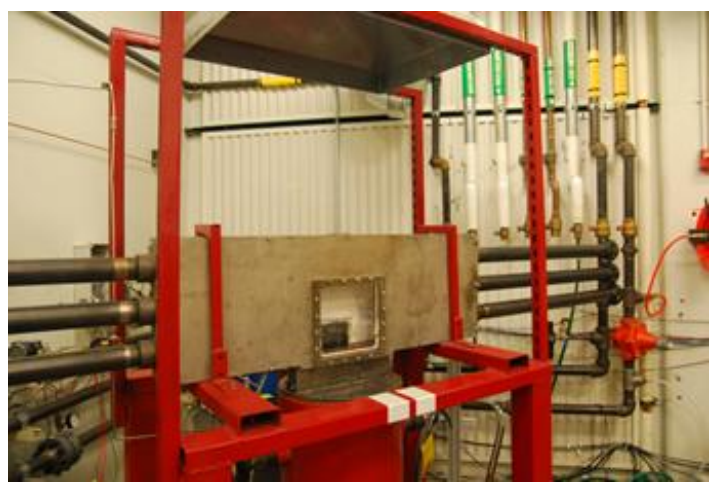

(a)

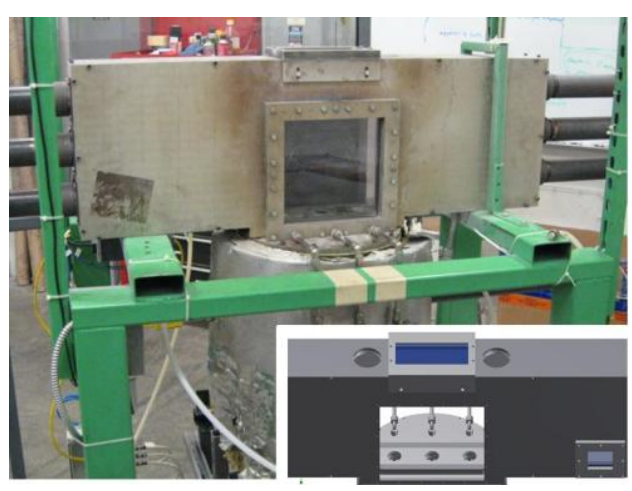

(b)

Figure 8 - Transverse forcing facilities in the unwrapped annular combustor configuration with high aspect ratio combustors and speaker tubes: (a) Experiment of O'Connor and Lieuwen [87], and (b) Experiment of Malanoski et al. [92]. 


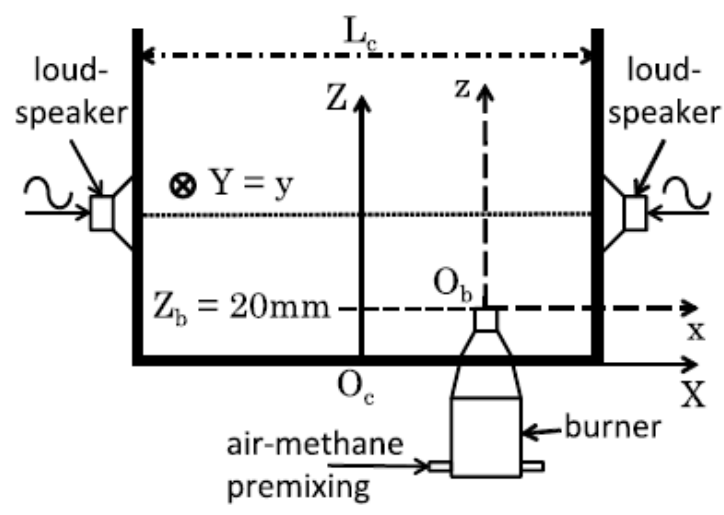

(a)

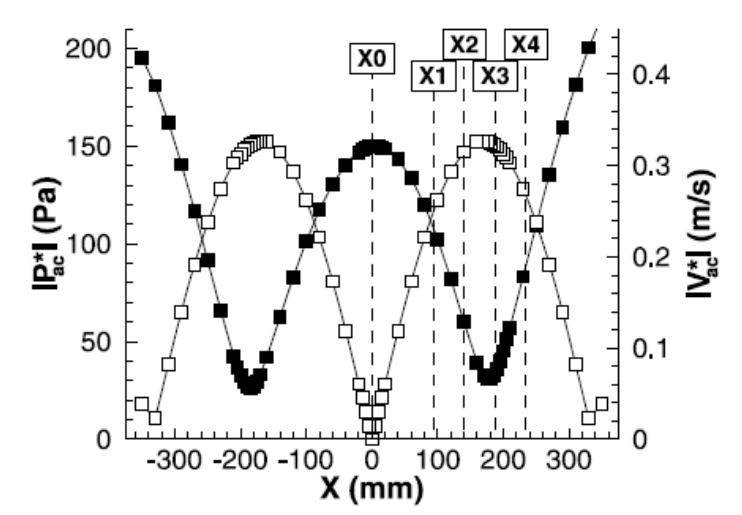

(b)

Figure 9 - (a) Acoustic forcing configuration and (b) acoustic field measurements for the transverse forcing facility of Lespinasse et al. [98]. Filled symbols are acoustic pressure measurements and open symbols are acoustic velocity measurements. 


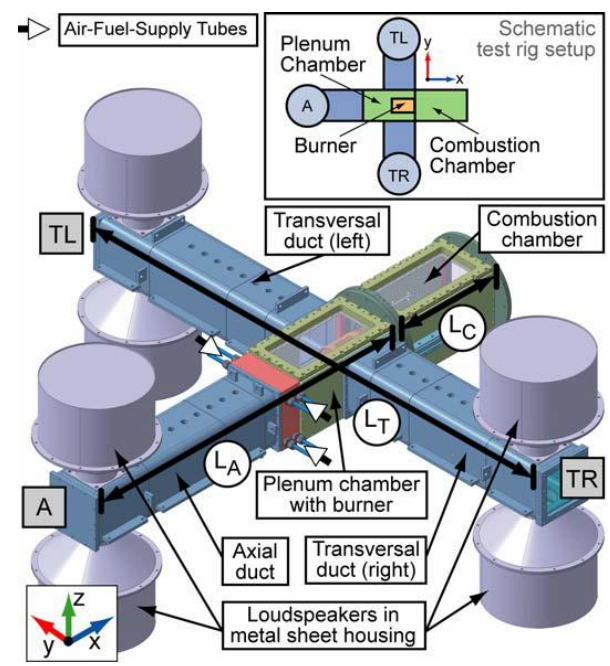

(a)

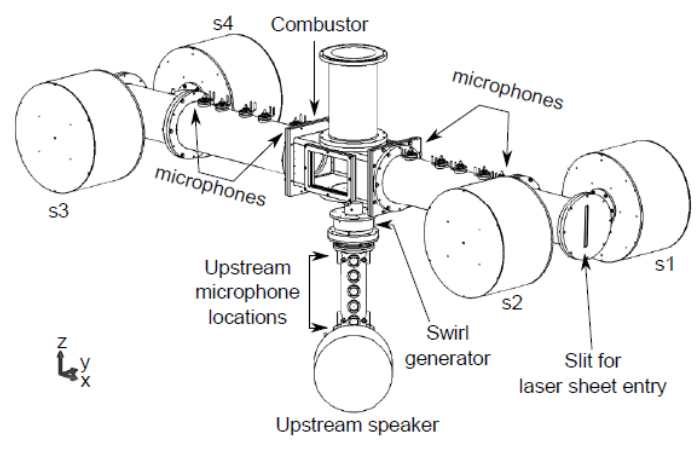

(b)

Figure 10 - T-shape transverse forcing experiments from (a) Hauser et al. [95] and (b) Saurabh and Paschereit [99], capable of both transverse and longitudinal forcing. 


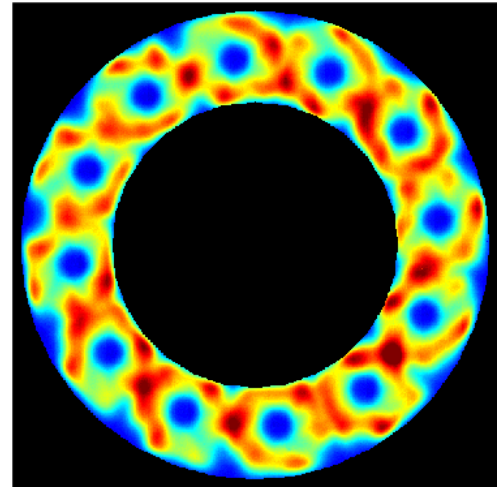

(a)

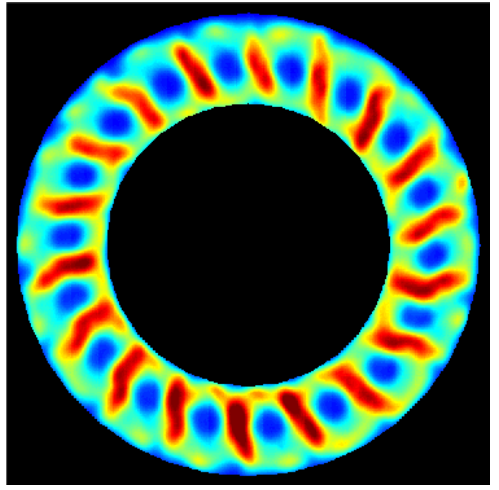

(b)

Figure 11 - Extent of flame interaction in an annular combustor measured via timeaverage $\mathrm{OH}^{*}$ chemiluminescence from an overhead view. Images show two nozzle spacings - (a) 2.33 and (b) 1.56 times the nozzle diameter - where all swirlers have a counterclockwise rotation direction. Reproduced from Worth and Dawson [102]. 


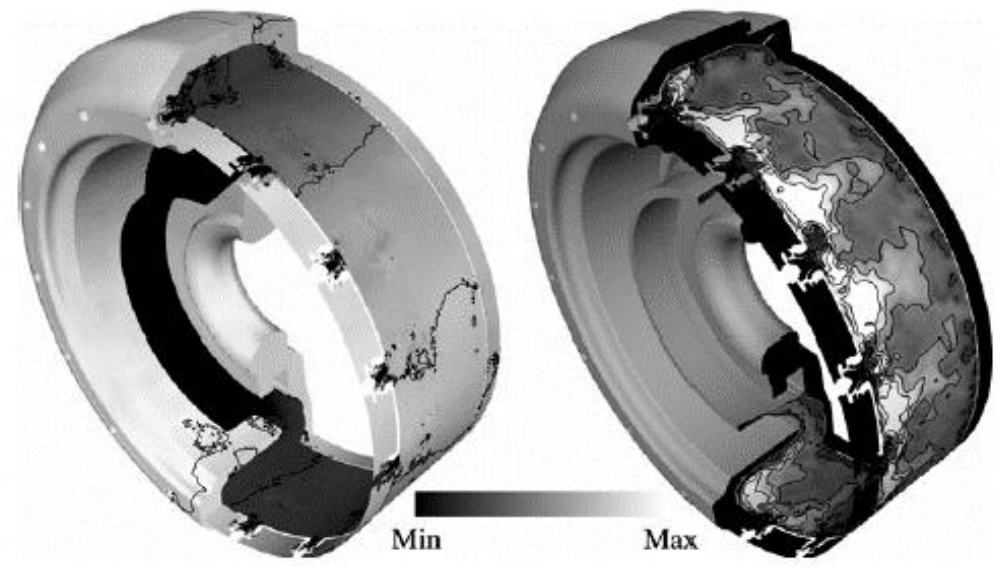

Figure 12 - Pressure (left) and temperature (right) fields from LES simulation of a full-annular combustor during azimuthal acoustic mode oscillations. Reproduced from Wolf et al. [134]. 


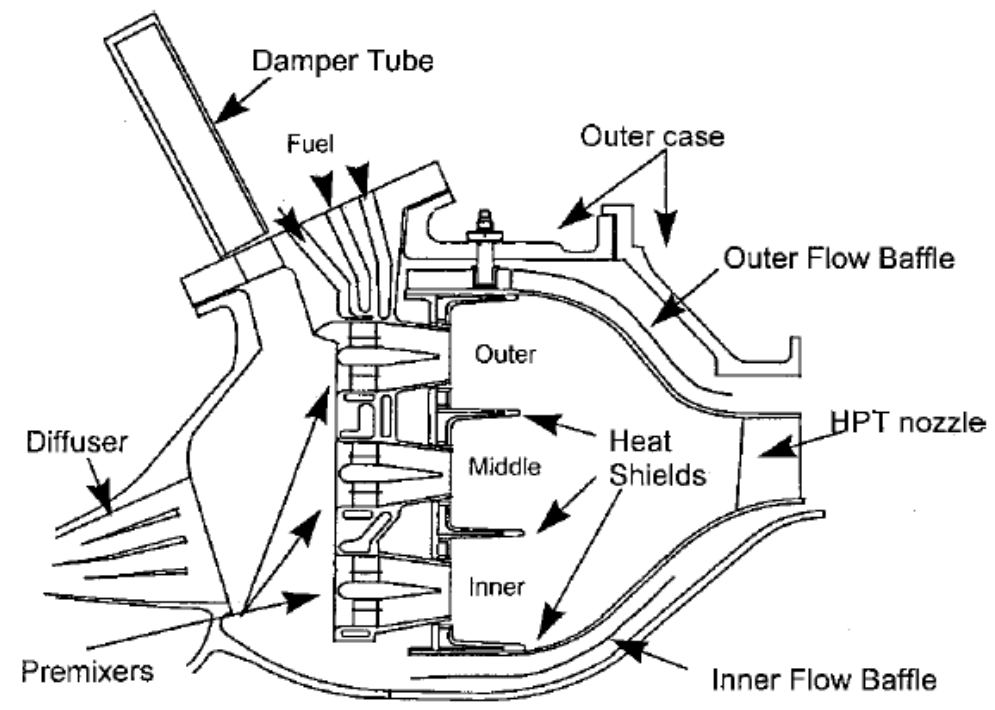

Figure 13 - General Electric LM6000 DLE combustor cross-section with damper tube in combustor plenum and circumferential baffles. Reproduced from Mongia $e t$ al. [78]. 


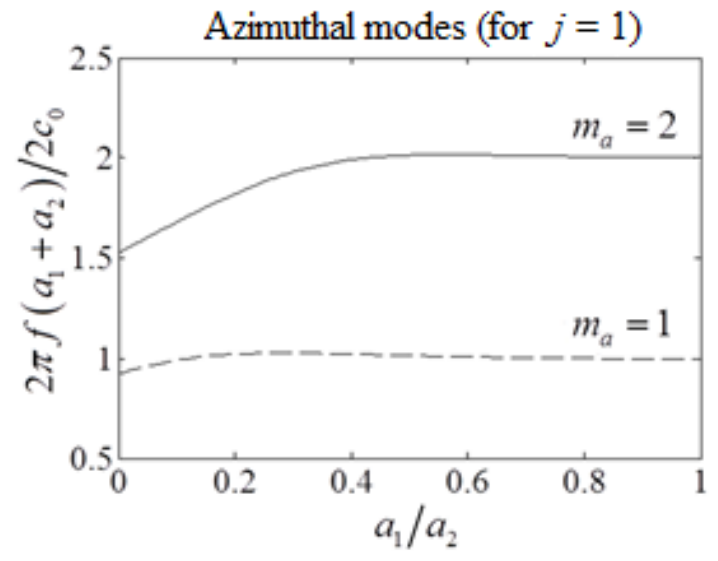

(a)

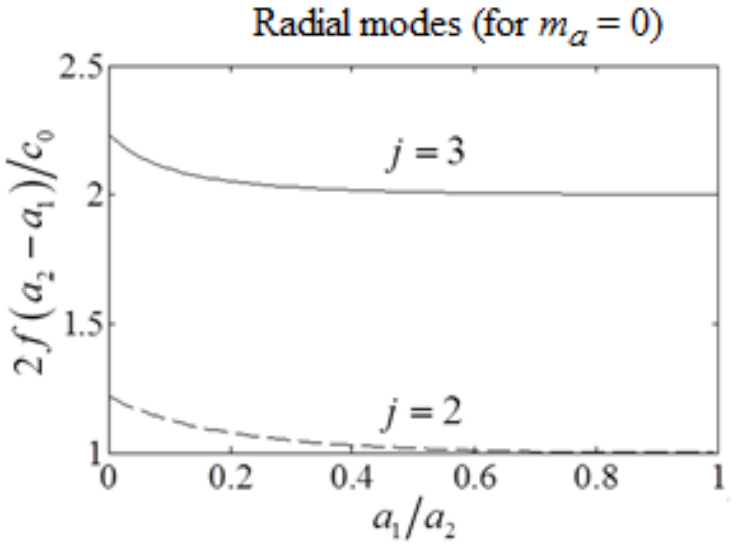

(b)

Figure 14 - Dependence of (a) azimuthal and (b) radial natural frequencies of annular geometry upon normalized gap width; following Lieuwen [195]. 


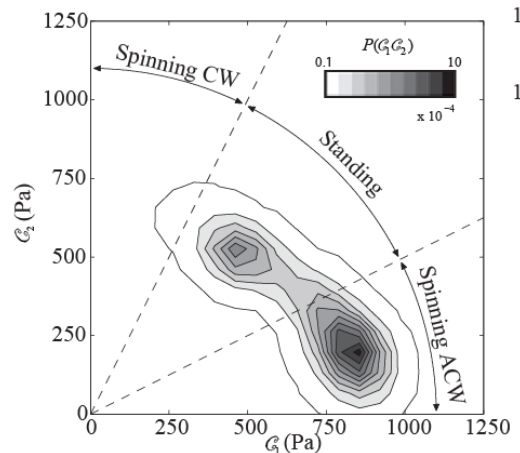

(a)

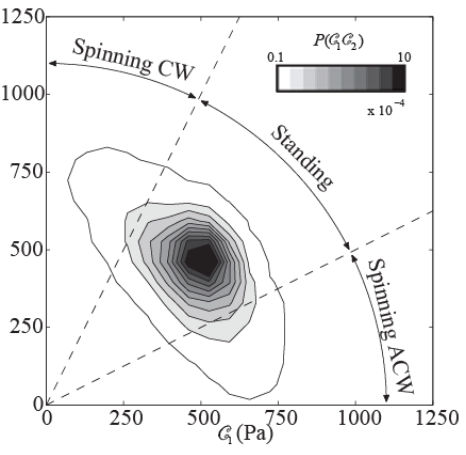

(b)

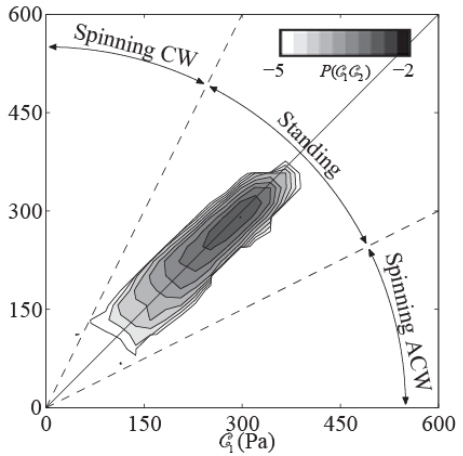

(c)

Figure 15 - Joint probability of forward and backward spinning wave amplitudes for an $m_{a}=1$ azimuthal instability in a model annular combustor with a) counterclockwise swirling nozzles and b) alternating swirl between clockwise and counterclockwise, and c) two baffles placed at $180^{\circ}$ angles around the annulus. Adapted from Worth and Dawson [102, 103]. 


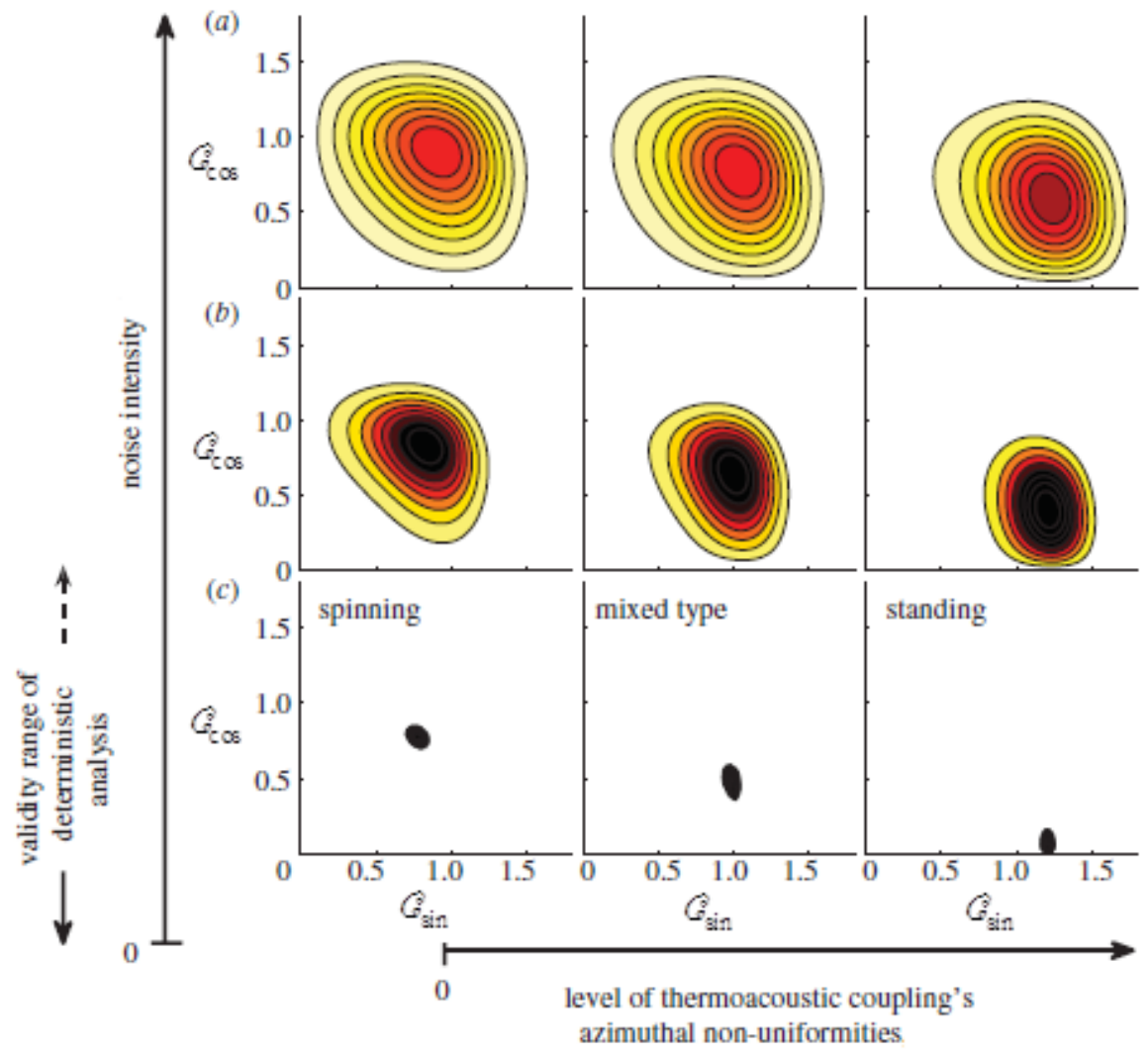

Figure 16 - Joint probability density functions of standing wave amplitudes in an azimuthally unstable annular combustor at three noise levels. Adapted from Noiray et al. [120]. 


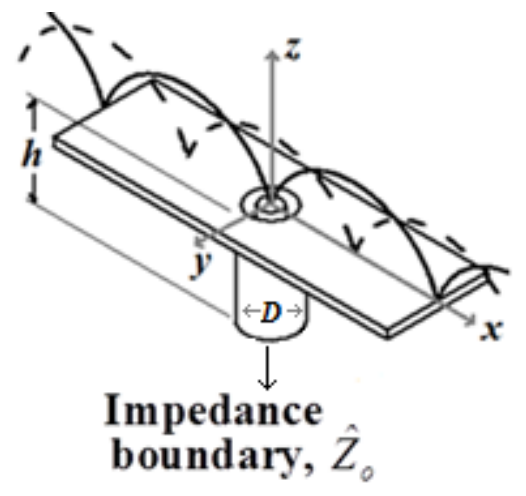

Figure 17 - Standing acoustic pressure and velocity fields shown relative to inflow nozzle. The $x$-coordinate corresponds to the azimuthal coordinate $\theta$ in the full annular combustor, where $x=R \theta$. 


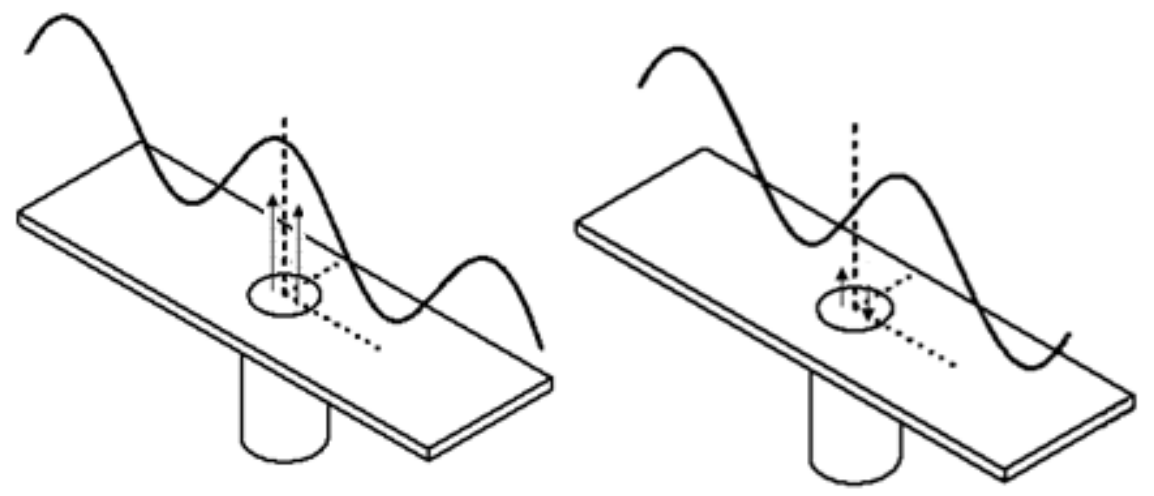

Figure 18 - Schematic of transverse pressure fluctuations as experienced by nozzle for pressure anti-node (a) and pressure node (b) forcing cases. 


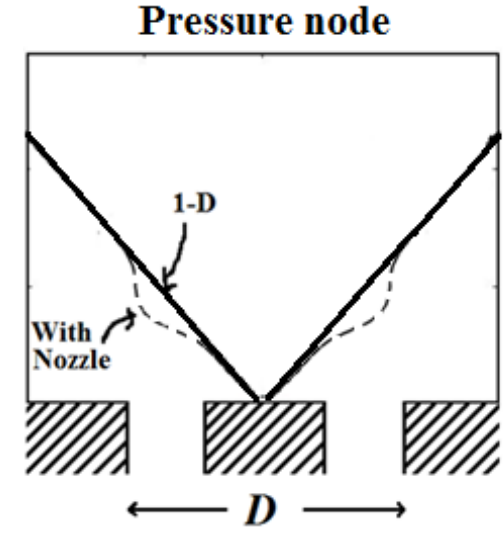

(a)

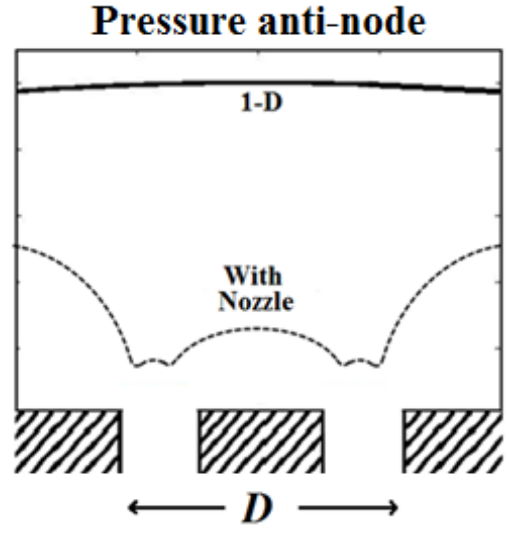

(b)

Figure 19 - Pressure amplitudes at the center plane $(y=0)$ for $D / \lambda \approx 0.04(400 \mathrm{~Hz})$ with (a) an anechoic nozzle for the pressure node case, and (b) pressure release condition at the nozzle for the pressure anti-node case. Reproduced from Blimbaum et al. [195]. 


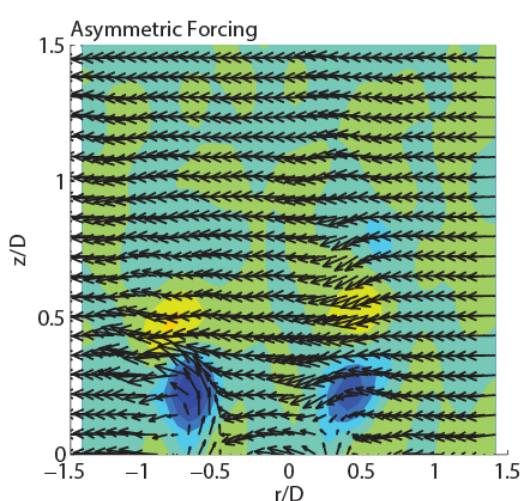

(a)

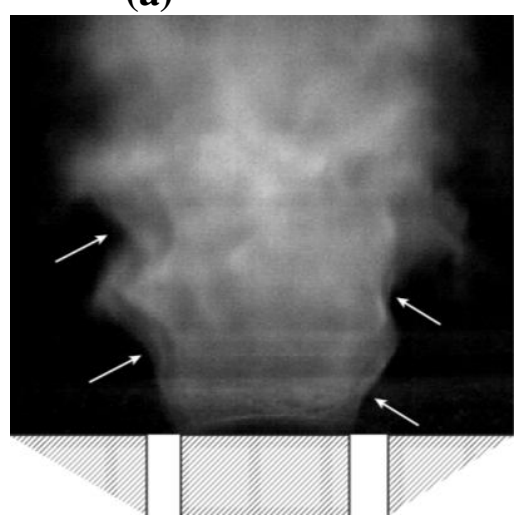

(c)

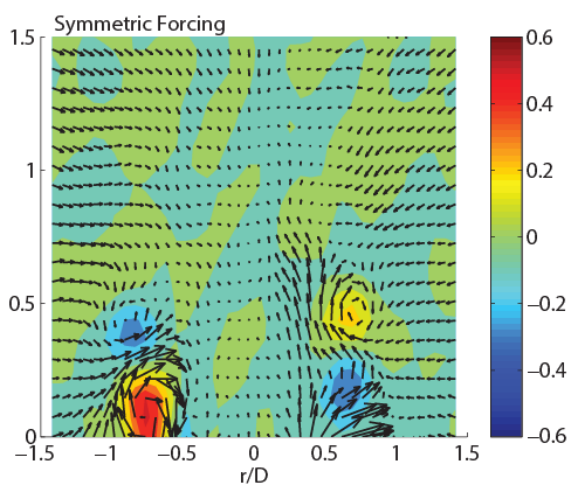

(b)

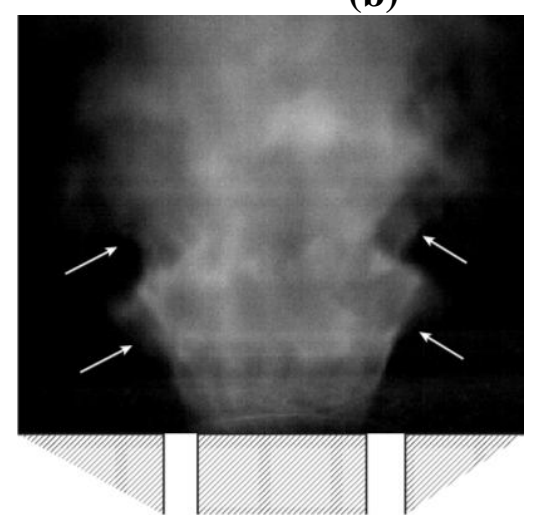

(d)

Figure 20 - Experimental results of PIV measurements of velocity (vectors) and normalized vorticity (color) fluctuation for $400 \mathrm{~Hz}$ asymmetric (a) and $400 \mathrm{~Hz}$ symmetric (b) forcing in non-reacting swirling flow at $u_{z, 0}=10 \mathrm{~m} / \mathrm{s}, S=0.85$. Experimental flame luminescence images of $400 \mathrm{~Hz}$ asymmetric (c) and $400 \mathrm{~Hz}$ symmetric (d) forcing of a flame in a swirling flow at $u_{z, 0}=10 \mathrm{~m} / \mathrm{s}, S=0.5$, and an equivalence ratio of 0.9 . Arrows point to the wrinkles resulting from c) helical and d) ring vortices. 


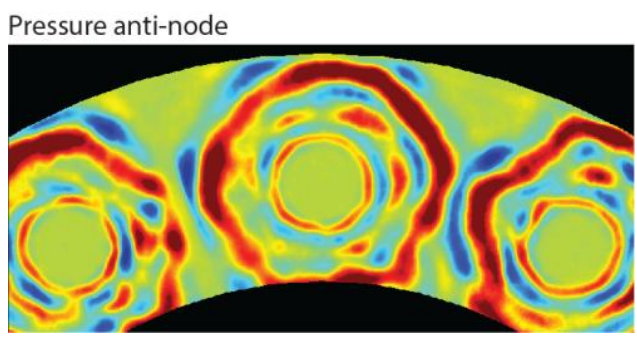

(a)

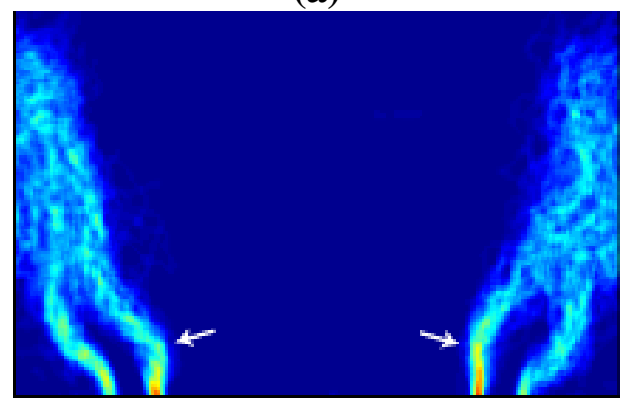

(c)

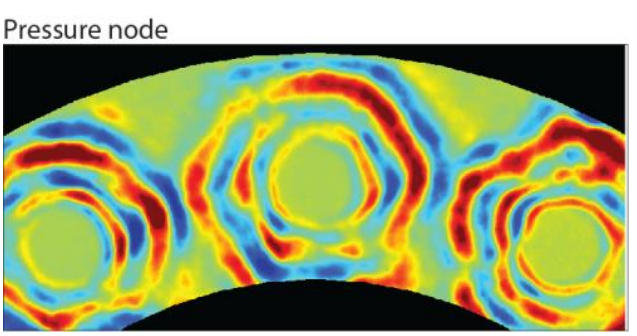

(b)

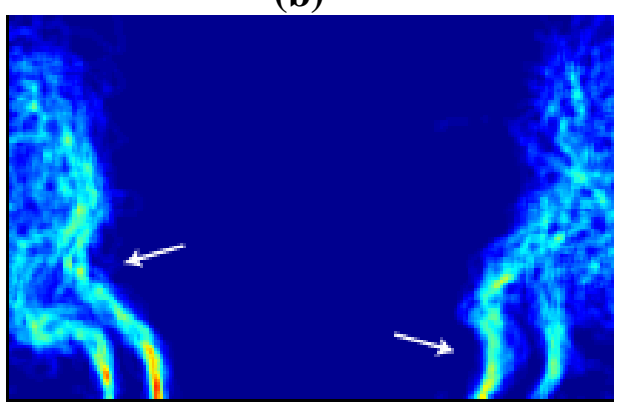

(d)

Figure 21 - Examples of symmetric and asymmetric flame oscillations in an annular combustor. Flames displaying (a) symmetric (ring vortex) and (b) asymmetric (helical vortex) response are shown using the fluctuation amplitude of $\mathrm{OH}^{*}$ chemiluminescence imaging from an overhead view [103]. In (c) and (d), OH-PLIF images of flames at pressure anti-nodes and velocity anti-nodes, respectively, with arrows showing similar flame wrinkling to Figure 20 [162]. 


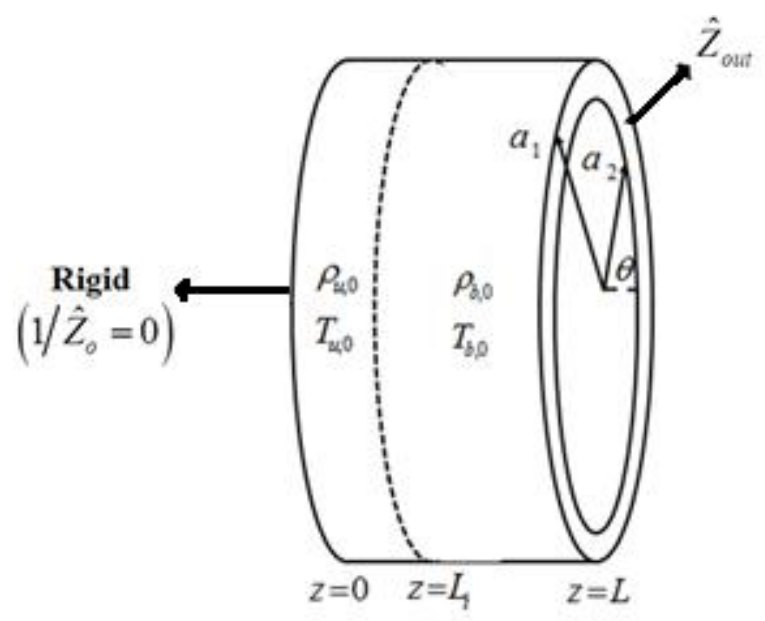

Figure 22 - Simplified representation of Figure 2 for a model thin annular combustor with a temperature discontinuity interface. 


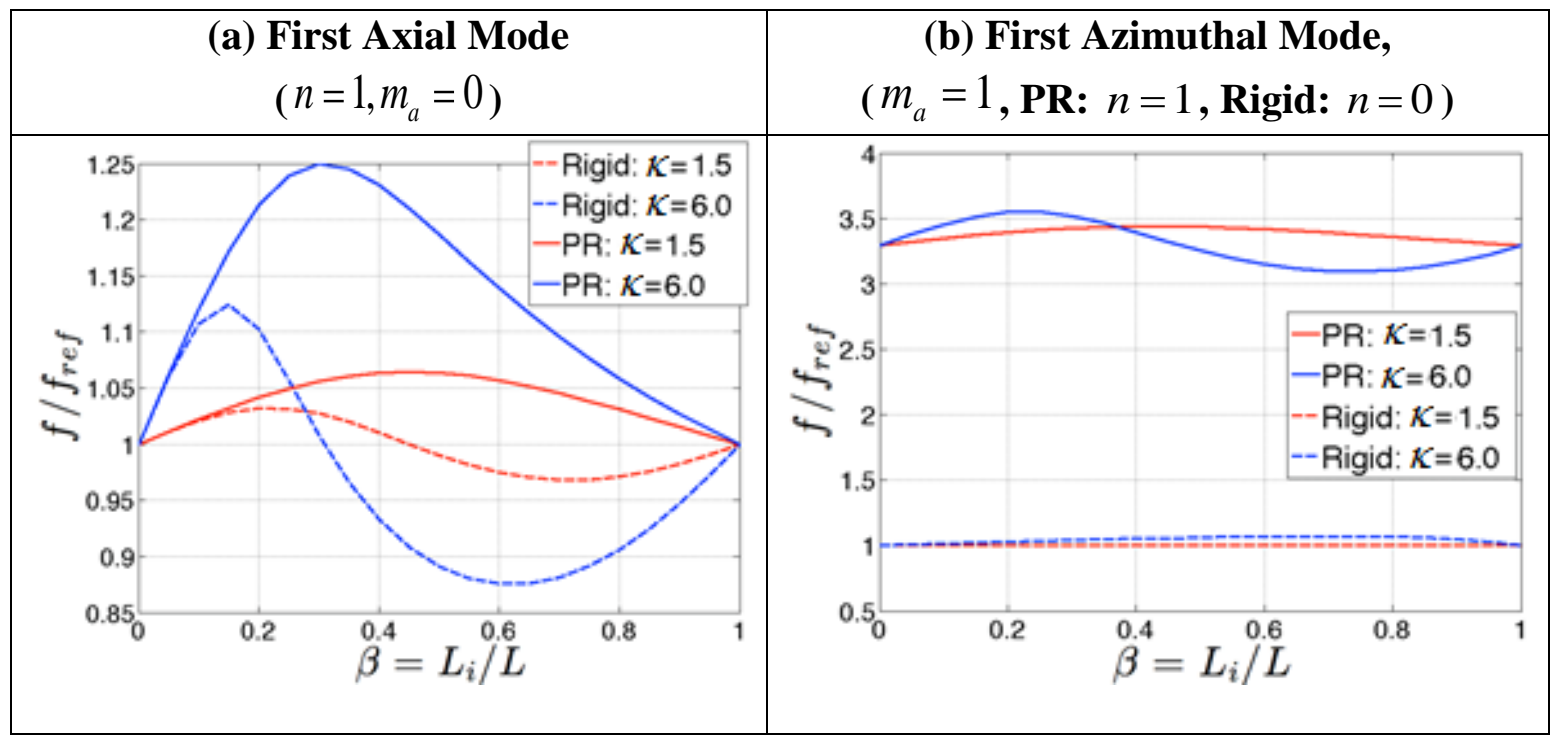

Figure 23 - Effect of azimuthal modes $(m)$ on the normalized natural frequency in a thin annular duct with $L / R=0.5$ and $\kappa=T_{b, 0} / T_{u, 0}$. "PR" denotes a pressure release outflow boundary condition. 


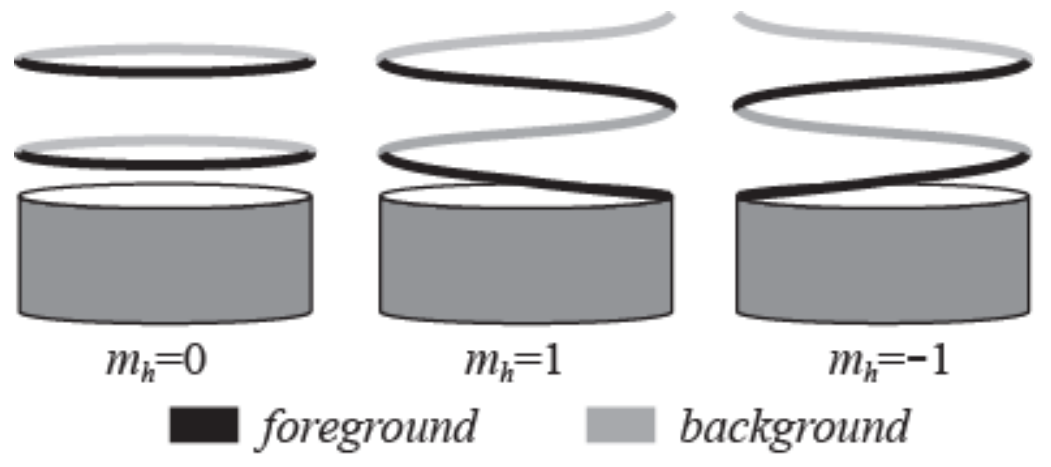

Figure 24 - Representation of modes for $m_{h}=0$ (ring vortices), $m_{h}>0$ (clockwisewinding helix), and $m_{h}<0$ (counter-clockwise-winding helix). 


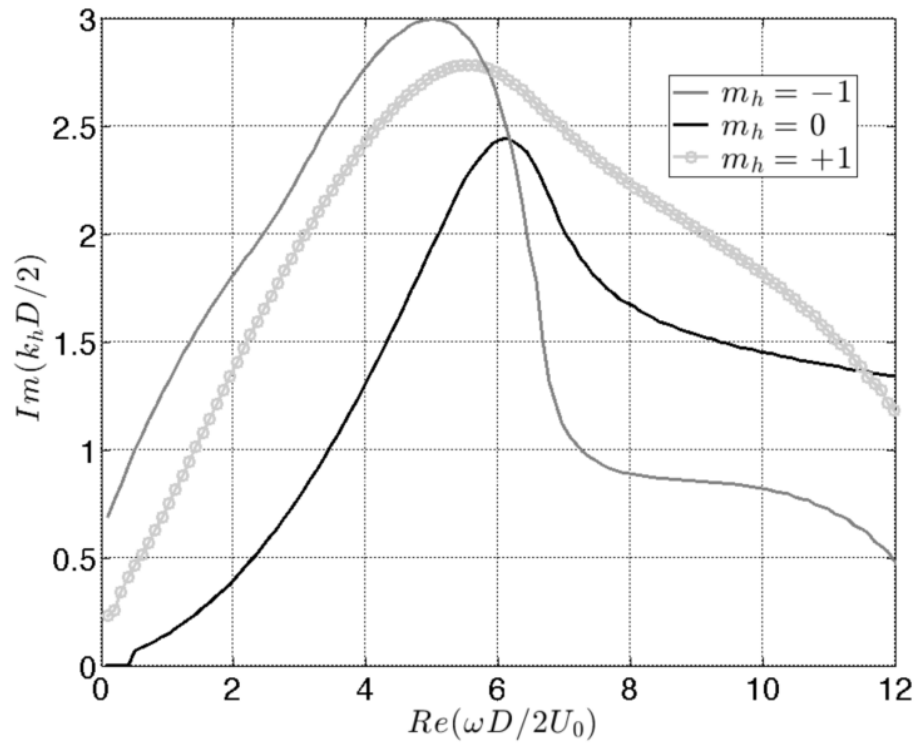

Figure 25 - Linear spatial growth rate of hydrodynamic modes in a swirling jet as a function of excitation frequency. Plot courtesy of Benjamin Emerson. 


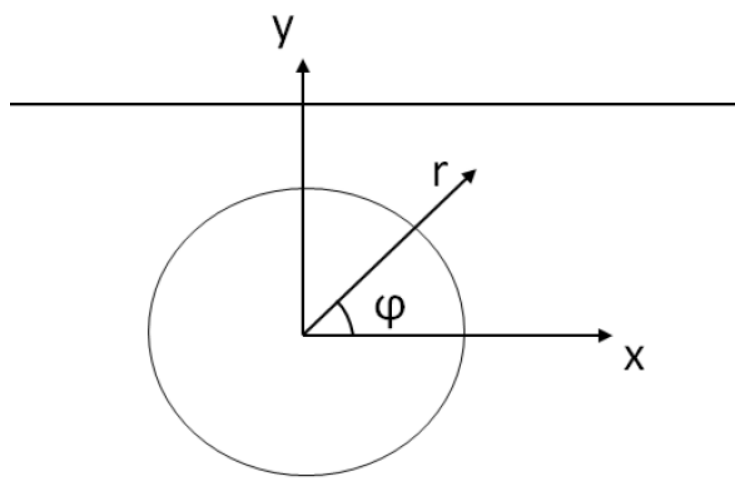

Figure 26 - Top-view of nozzle showing Cartesian and cylindrical polar coordinate systems with respect to the center of the nozzle. 


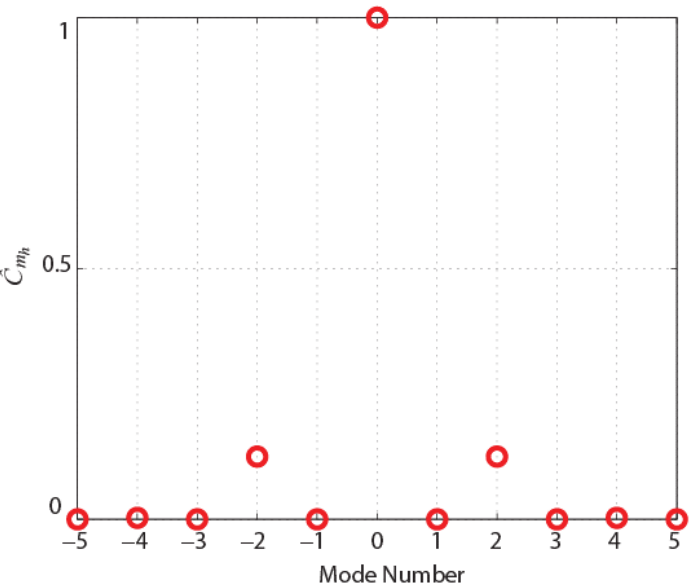

(a)

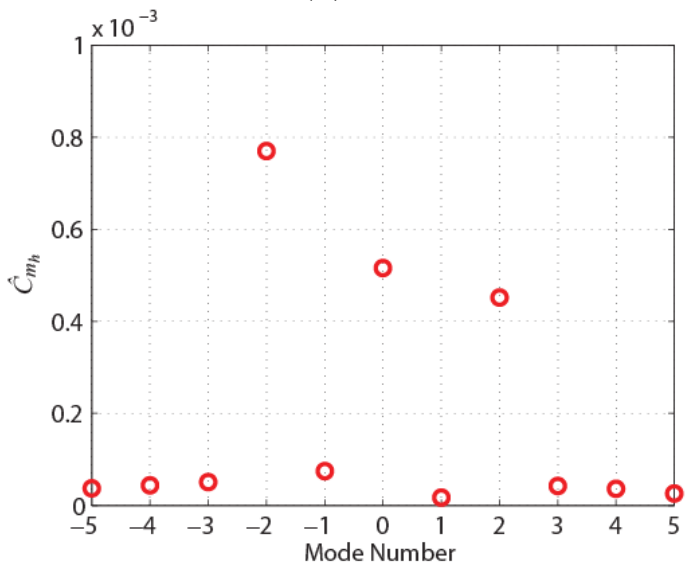

(c)

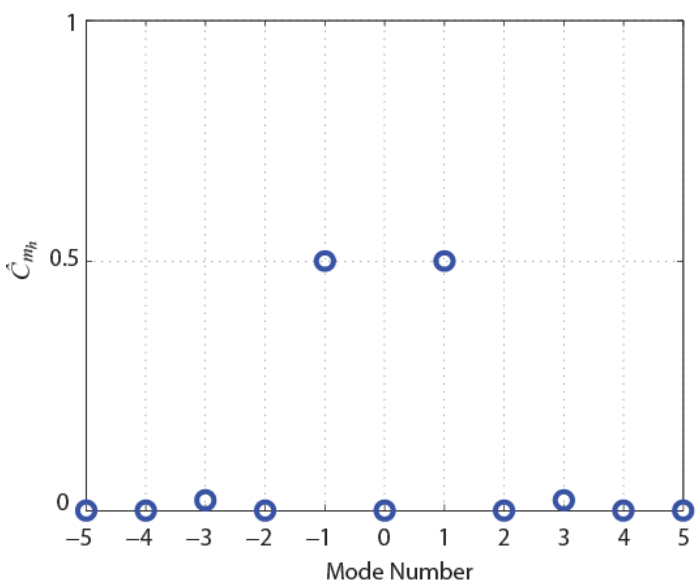

(b)

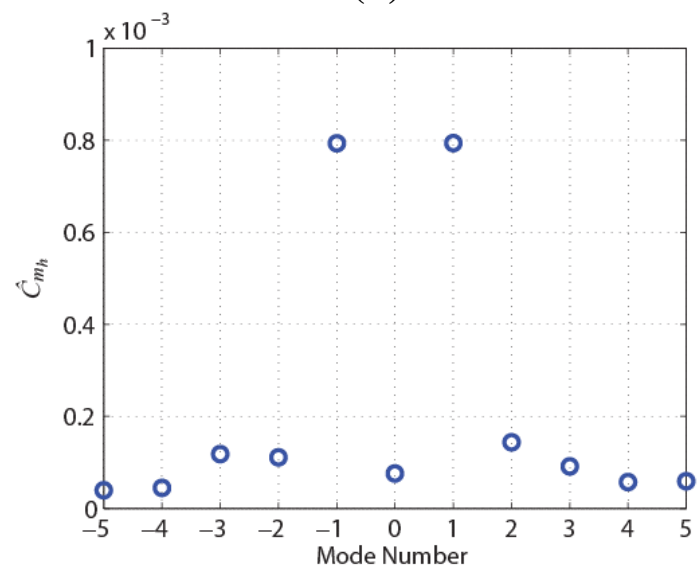

(d)

Figure 27 - Amplitude of helical modes excited by (a) symmetric forcing and (b) asymmetric acoustic forcing. Illustrative results from transversely forced cold-flow experiment by O'Connor and Lieuwen [87] showing modal amplitudes for $1200 \mathrm{~Hz}$ (c) symmetric forcing and (d) asymmetric forcing. 
(a)

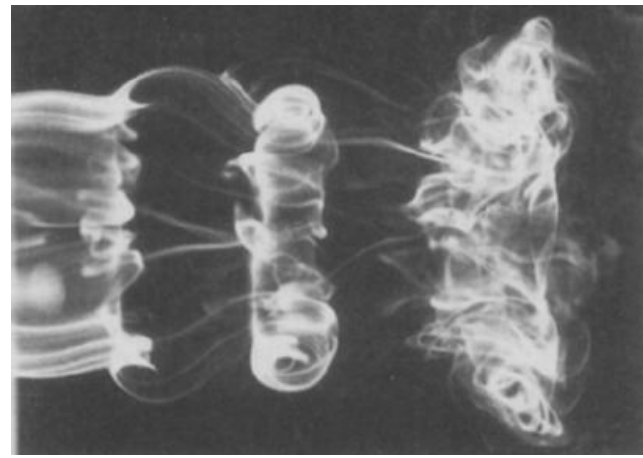

(b)

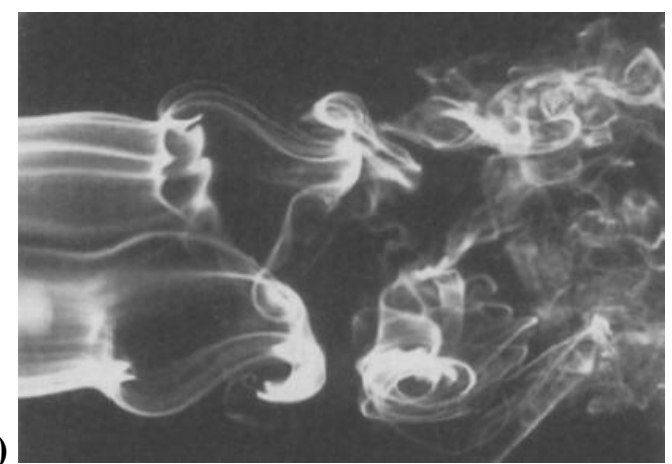

Figure 28 - Response of a circular jet to (a) symmetric and (b) asymmetric (forcing of the $m_{h}= \pm 1$ modes) acoustic forcing around the circumference of the jet. Images reproduced from Kusek et al. [250]. 


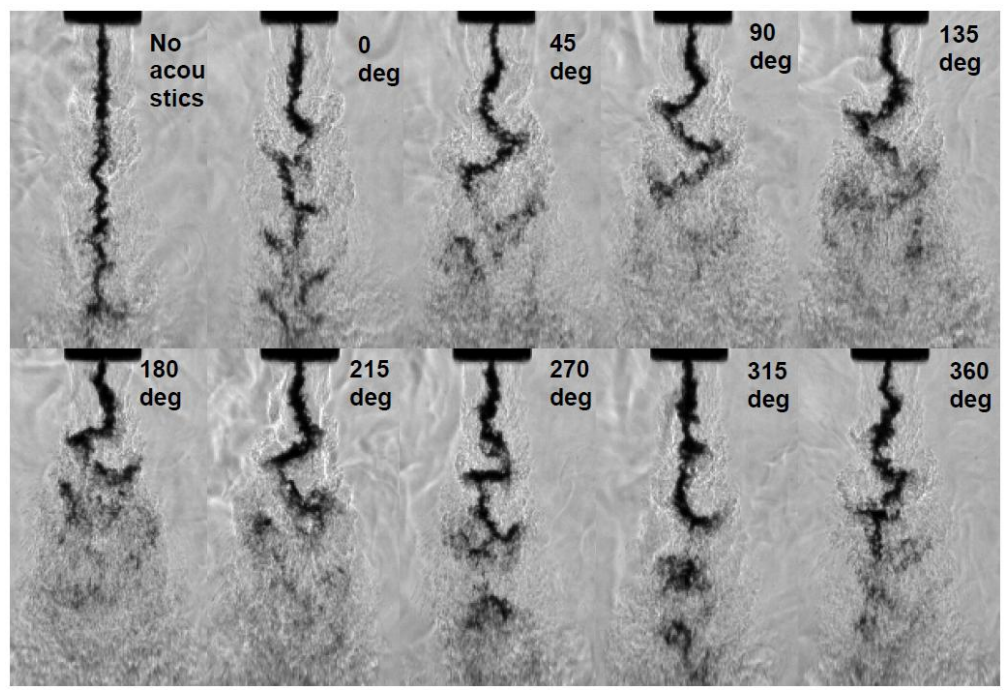

Figure 29. Response of a liquid coaxial jet in a transverse acoustic at a range of phases between the speakers at either end of a transverse forcing facility [271]. 


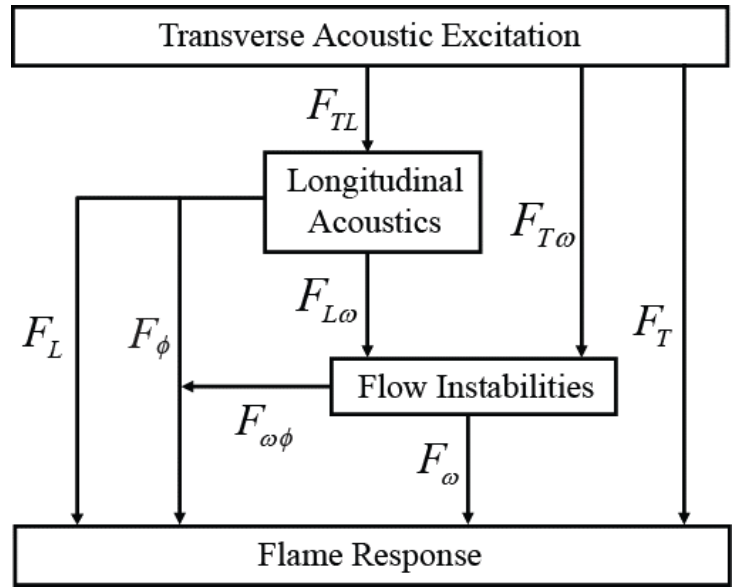

Figure 30 - Flame coupling pathways in transversely excited flames. 


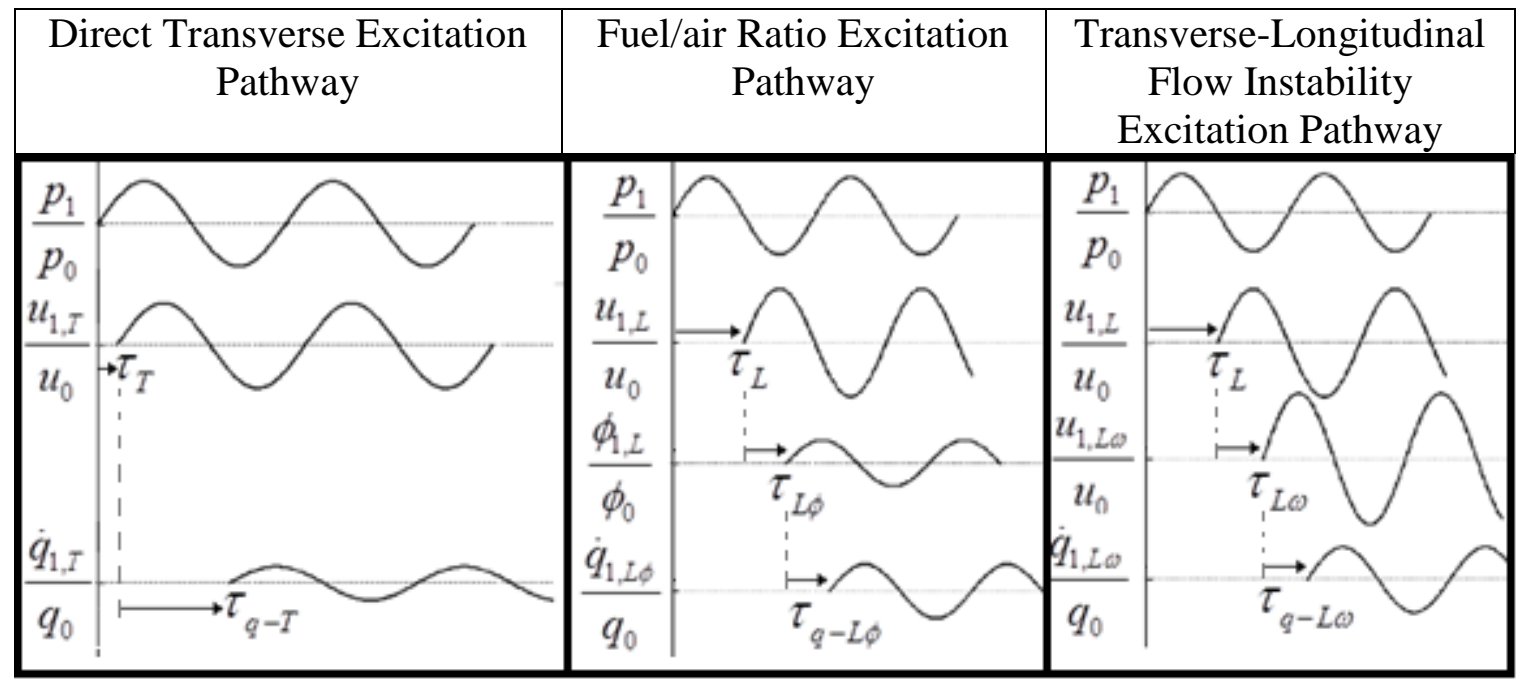

(a)

(b)

(c)

Figure 31 - Time-delays between the unsteady pressure and intermediate processes affecting the heat release: (a) Effect of transverse velocity oscillations, (b) Effect of fuel/air ratio oscillations from induced longitudinal velocity oscillations, (c) Effect of flow instabilities. 


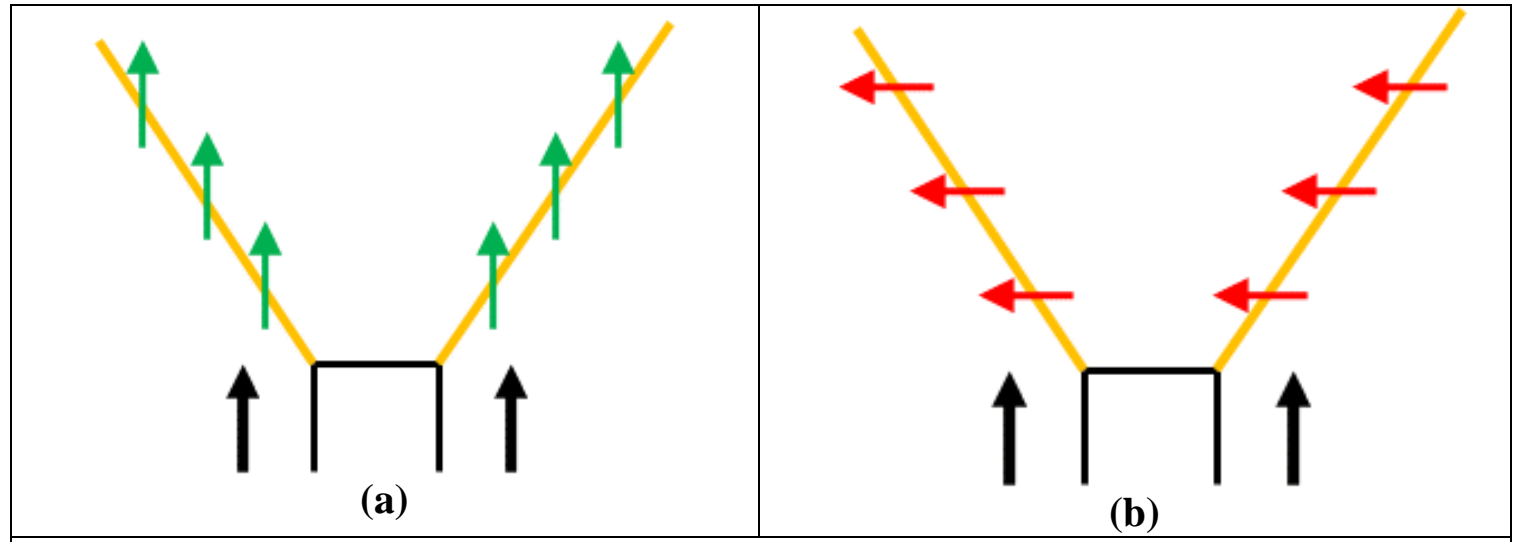

Figure 32 - Velocity fluctuations during (a) longitudinal forcing; (b) transverse forcing. 


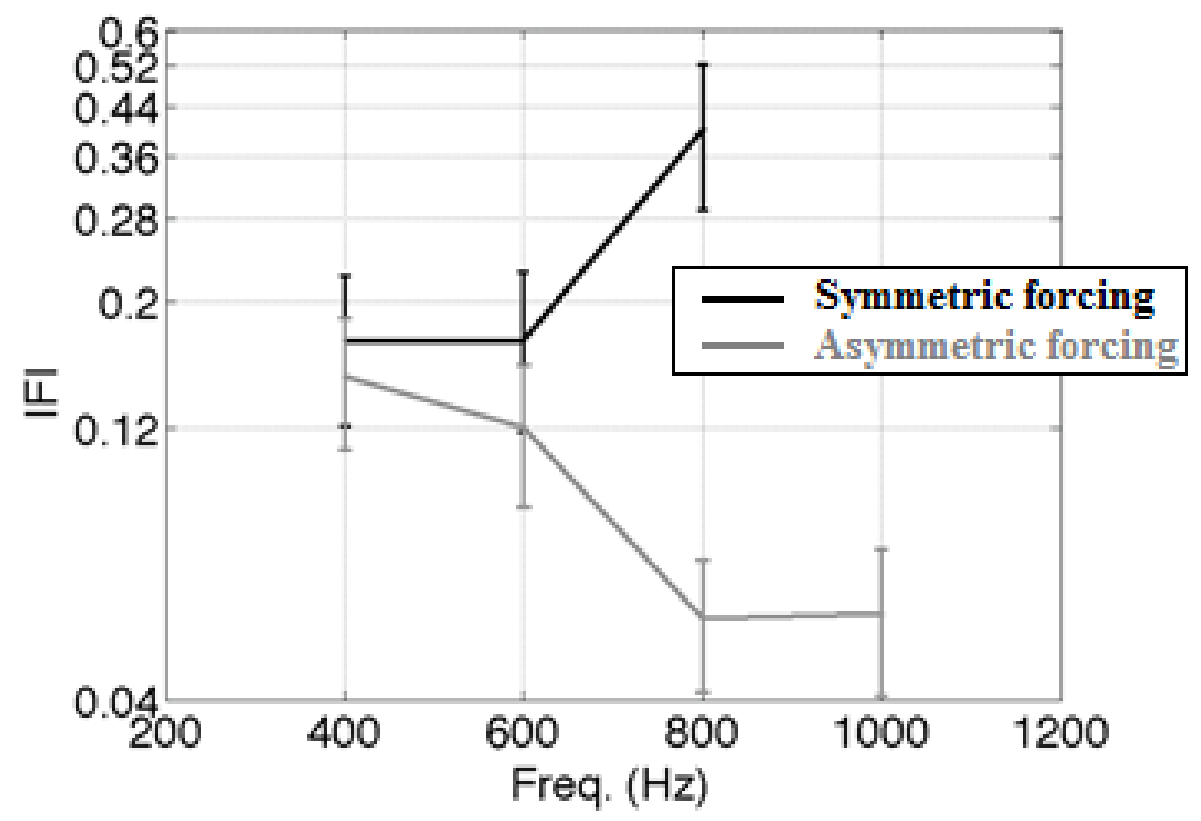

Figure 33 - Flame transfer function $(F)$ amplitude comparison between symmetric (black) and asymmetric (gray) forcing. Data reproduced from Acharya et al. [128]. 


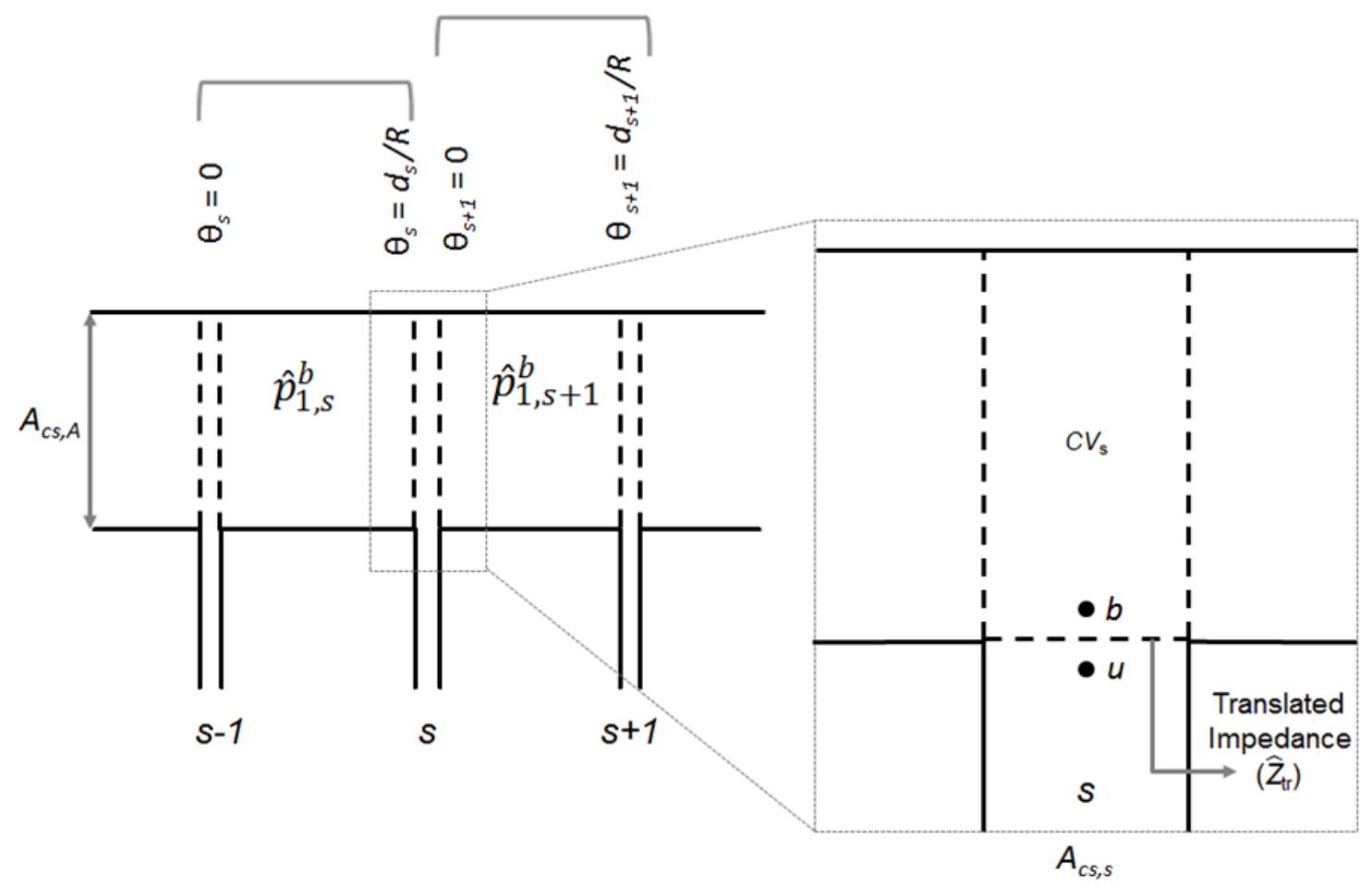

Figure 34 - Cut-section side view along the circumferential direction, showing the control volume and the coordinate system. 


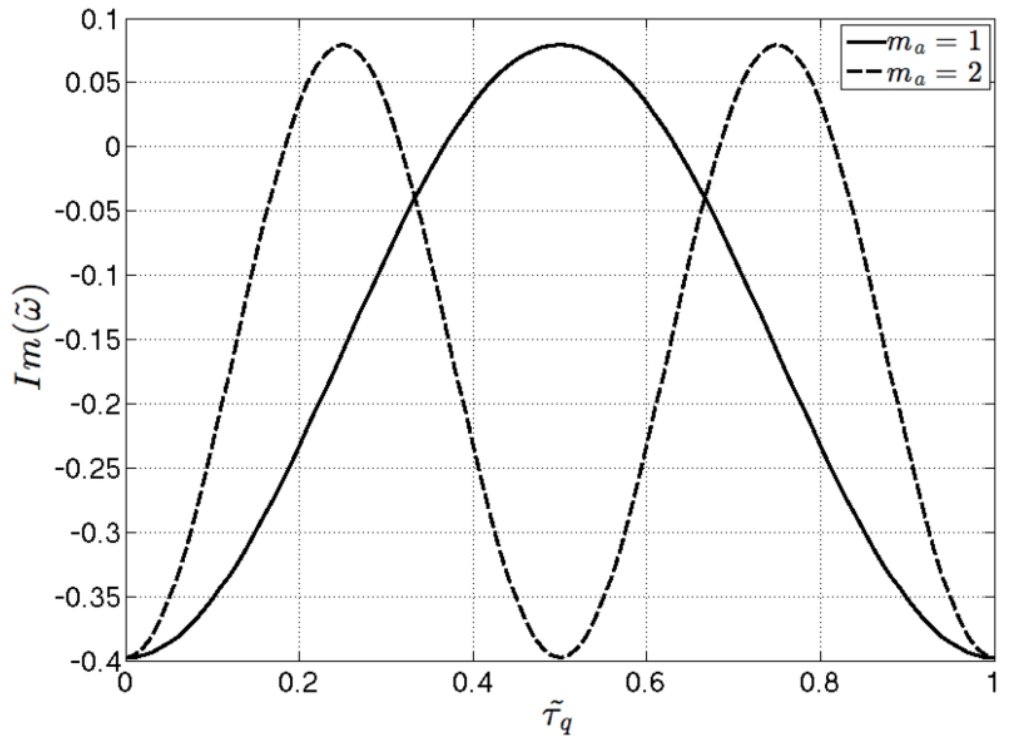

Figure 35 - Variation of instability growth rate for the 1 case for the fundamental frequency $m_{a}=1$ and the second mode $m_{a}=2$, with the time-delay parameter $\tilde{\tau}_{q}$ for $n=1.5$. 


\section{Tables}

Table 1. Overview of transverse instability studies.

Experimental Studies

\begin{tabular}{|c|c|c|c|c|}
\hline $\begin{array}{l}\text { Combustor } \\
\text { geometry }\end{array}$ & $\begin{array}{c}\text { Flame } \\
\text { Characteristics } \\
\end{array}$ & $\begin{array}{c}\text { Frequency } \\
\text { Range }(\mathrm{Hz}) \\
\end{array}$ & Authors & Measurements \\
\hline rectangular & $\begin{array}{l}\text { bluff-body } \\
\text { stabilized } \\
\text { premixed }\end{array}$ & $3500-4500$ & $\begin{array}{l}\text { Rogers and } \\
\text { Marble [9] }\end{array}$ & $\begin{array}{l}\text { pressure } \\
\text { visible imaging } \\
\text { schlieren }\end{array}$ \\
\hline rectangular & $\begin{array}{l}\text { bluff-body } \\
\text { stabilized } \\
\text { premixed }\end{array}$ & 4000 & $\begin{array}{l}\text { Kaskan and } \\
\text { Noreen [11] }\end{array}$ & $\begin{array}{l}\text { pressure } \\
\text { visible imaging }\end{array}$ \\
\hline rectangular & $\begin{array}{l}\text { bluff-body } \\
\text { stabilized } \\
\text { premixed }\end{array}$ & $1800-5400$ & Elias [10] & $\begin{array}{l}\text { pressure } \\
\text { visible imaging }\end{array}$ \\
\hline cylindrical & $\begin{array}{l}\text { bluff-body } \\
\text { stabilized } \\
\text { partially } \\
\text { premixed }\end{array}$ & $3300-3500$ & $\begin{array}{l}\text { Blackshear et } \\
\text { al. [81] }\end{array}$ & pressure \\
\hline $\begin{array}{l}\text { cylindrical } \\
\text { tube }\end{array}$ & $\begin{array}{l}\text { swirl stabilized } \\
\text { premixed }\end{array}$ & $2000-3500$ & $\begin{array}{l}\text { Sattelmayer } \\
\text { and } \\
\text { coworkers } \\
{[82-84]}\end{array}$ & $\begin{array}{l}\text { pressure } \\
\text { Mie scattering } \\
\text { PIV } \\
\mathrm{OH}^{*} \\
\text { chemiluminescence }\end{array}$ \\
\hline $\begin{array}{l}\text { High aspect } \\
\text { ratio, } \\
\text { rectangular } \\
\text { transverse } \\
\text { forcing } \\
\text { facility }\end{array}$ & $\begin{array}{l}\text { swirl stabilized } \\
\text { premixed }\end{array}$ & $400-1800$ & $\begin{array}{l}\text { O’Connor } \\
\text { and } \\
\text { coworkers } \\
{[76,85-90]}\end{array}$ & $\begin{array}{l}\text { pressure } \\
\text { PIV } \\
\text { OH PLIF } \\
\text { visible imaging }\end{array}$ \\
\hline $\begin{array}{l}\text { High aspect } \\
\text { ratio } \\
\text { rectangular } \\
\text { transverse } \\
\text { forcing } \\
\text { facility }\end{array}$ & $\begin{array}{l}\text { swirl stabilized } \\
\text { premixed/partiall } \\
\text { y premixed }\end{array}$ & $400-1200$ & $\begin{array}{l}\text { Malanoski } \\
\text { and } \\
\text { coworkers } \\
{[91,92]}\end{array}$ & $\begin{array}{l}\text { PIV } \\
\mathrm{CH}^{*} \\
\text { chemiluminescence }\end{array}$ \\
\hline $\begin{array}{l}\text { High aspect } \\
\text { ratio, } \\
\text { rectangular } \\
\text { transverse } \\
\text { forcing } \\
\text { facility }\end{array}$ & $\begin{array}{l}\text { bluff-body } \\
\text { stabilized } \\
\text { partially } \\
\text { premixed }\end{array}$ & 450 & $\begin{array}{l}\text { Emerson et } \\
\text { al. }[93,94]\end{array}$ & $\begin{array}{l}\text { PIV } \\
\text { Luminescence }\end{array}$ \\
\hline transverse & swirl stabilized & $0-200$ & Hauser and & pressure \\
\hline
\end{tabular}




\begin{tabular}{|l|l|l|l|l|}
\hline $\begin{array}{l}\text { and axial } \\
\text { forcing } \\
\text { facility }\end{array}$ & premixed & $\begin{array}{l}\text { coworkers } \\
{[95,96]}\end{array}$ & $\begin{array}{l}\text { CTA } \\
\text { OH* } \\
\text { chemiluminescence }\end{array}$ \\
\hline $\begin{array}{l}\text { high aspect } \\
\text { ratio } \\
\text { rectangular } \\
\text { transverse } \\
\text { forcing } \\
\text { facility }\end{array}$ & $\begin{array}{l}\text { bluff-body } \\
\text { stabilized } \\
\text { premixed }\end{array}$ & $10-1100$ & $\begin{array}{l}\text { Lespinasse } \\
\text { and } \\
\text { coworkers } \\
{[97,98]}\end{array}$ & $\begin{array}{l}\text { pressure } \\
\text { Mie scattering } \\
\text { PIV }\end{array}$ \\
\hline $\begin{array}{l}\text { transverse } \\
\text { and axial } \\
\text { forcing } \\
\text { facility }\end{array}$ & $\begin{array}{l}\text { swirl stabilized } \\
\text { premixed }\end{array}$ & $0-200$ & $\begin{array}{l}\text { Saurabh and } \\
\text { Paschereit } \\
{[99,100]}\end{array}$ & $\begin{array}{l}\text { pressure } \\
\text { PIV }\end{array}$ \\
\hline $\begin{array}{l}\text { annular } \\
\text { combustor }\end{array}$ & $\begin{array}{l}\text { swirl stabilized } \\
\text { premixed }\end{array}$ & $1700-1800$ & $\begin{array}{l}\text { Worth and } \\
\text { Dawson } \\
{[101-103]}\end{array}$ & $\begin{array}{l}\text { pressure } \\
\text { OH* } \\
\text { chemiluminescence } \\
\text { OH PLIF }\end{array}$ \\
\hline $\begin{array}{l}\text { annular } \\
\text { combustor }\end{array}$ & $\begin{array}{l}\text { swirl stabilized } \\
\text { premixed }\end{array}$ & $0-2500$ & $\begin{array}{l}\text { Bourgouin } \\
\text { and } \\
\text { coworkers } \\
{[104,105]}\end{array}$ & $\begin{array}{l}\text { pressure } \\
\text { luminescence }\end{array}$ \\
\hline $\begin{array}{l}\text { annular } \\
\text { combustor }\end{array}$ & Rijke tubes & $0-2000$ & $\begin{array}{l}\text { Moeck and } \\
\text { Paschereit } \\
{[106,107]}\end{array}$ & Pressure \\
\hline
\end{tabular}

Modeling Studies

\begin{tabular}{|c|c|c|c|}
\hline Model output & $\begin{array}{c}\text { Model } \\
\text { Dimension }\end{array}$ & Method & Authors \\
\hline Non-linear stability & $2 \mathrm{D}$ & Normal mode/Galerkin & $\begin{array}{l}\text { Maslen and } \\
\text { Moore [108] }\end{array}$ \\
\hline Mode identification & $1 \mathrm{D}$ & Normal mode/Galerkin & $\begin{array}{l}\text { Ghirardo and } \\
\text { Juniper [109] }\end{array}$ \\
\hline Non-linear instability & $2 \mathrm{D}$ & Normal mode Galerkin & $\begin{array}{l}\text { Burnley and } \\
\text { Culick [110] }\end{array}$ \\
\hline Linear stability & $3 \mathrm{D}$ & Normal mode/Galerkin & $\begin{array}{l}\text { Sensiau et al. } \\
\text { [111] }\end{array}$ \\
\hline Mode identification & $2 \mathrm{D}$ & Normal mode/Galerkin & $\begin{array}{l}\text { Polifke and } \\
\text { coworkers } \\
{[112,113]}\end{array}$ \\
\hline Non-linear stability & $2 \mathrm{D}$ & Normal mode Galerkin & $\begin{array}{l}\text { Yang and } \\
\text { Culick [114] }\end{array}$ \\
\hline Mode identification & $2 \mathrm{D}$ & Normal mode Galerkin & $\begin{array}{l}\text { Krebs and } \\
\text { coworkers } \\
{[115]}\end{array}$ \\
\hline Non-linear stability & $3 \mathrm{D}$ & Finite element & Pankiewitz \\
\hline
\end{tabular}




\begin{tabular}{|c|c|c|c|}
\hline & & & $\begin{array}{l}\text { and } \\
\text { Sattelmayer } \\
{[116]}\end{array}$ \\
\hline Mode identification & $3 \mathrm{D}$ & Finite element & $\begin{array}{l}\text { Nicoud and } \\
\text { coworkers } \\
{[111,117]}\end{array}$ \\
\hline Mode identification & $3 \mathrm{D}$ & Finite element & $\begin{array}{l}\text { Campa and } \\
\text { coworkers } \\
{[118,119]}\end{array}$ \\
\hline $\begin{array}{l}\text { Linear and non-linear } \\
\text { stability, mode } \\
\text { identification }\end{array}$ & $3 \mathrm{D}$ & Normal mode/Galerkin & $\begin{array}{l}\text { Schuermans } \\
\text { and coworkers } \\
{[120-122]}\end{array}$ \\
\hline Linear stability & $2 \mathrm{D}$ & Normal mode/Galerkin & $\begin{array}{l}\text { Parmentier } e t \\
\text { al. [123] }\end{array}$ \\
\hline Linear stability & $3 \mathrm{D}$ & Normal mode/Galerkin & $\begin{array}{l}\text { You et al. } \\
{[124]}\end{array}$ \\
\hline Linear stability & $3 \mathrm{D}$ & Normal mode/Galerkin & $\begin{array}{l}\text { Dowling and } \\
\text { Stow [125] }\end{array}$ \\
\hline $\begin{array}{l}\text { Flame dynamics/ } \\
\text { Instability identification }\end{array}$ & $3 \mathrm{D}$ & Level-set/normal mode & $\begin{array}{l}\text { Graham and } \\
\text { Dowling } \\
{[126]}\end{array}$ \\
\hline Flame dynamics & $3 \mathrm{D}$ & Level-set & $\begin{array}{l}\text { Acharya and } \\
\text { coworkers } \\
{[127-130]}\end{array}$ \\
\hline Flame dynamics & $2 \mathrm{D}$ & URANS & $\begin{array}{l}\text { Lee and Cant } \\
\text { [131] }\end{array}$ \\
\hline $\begin{array}{l}\text { Flame dynamics/ } \\
\text { Instability identification }\end{array}$ & $3 \mathrm{D}$ & LES/normal mode & $\begin{array}{l}\text { Staffelbach } \\
\text { and coworkers } \\
{[75,79,132-} \\
136]\end{array}$ \\
\hline Flame dynamics & $3 \mathrm{D}$ & LES & $\begin{array}{l}\text { Fureby and } \\
\text { coworkers } \\
{[137,138]} \\
\end{array}$ \\
\hline Flame dynamics & $3 \mathrm{D}$ & LES & $\begin{array}{l}\text { Zellhuber and } \\
\text { coworkers [6, } \\
139]\end{array}$ \\
\hline Flame dynamics & $3 \mathrm{D}$ & LES & $\begin{array}{l}\text { Selle et al. } \\
{[140]}\end{array}$ \\
\hline Flame dynamics & $3 \mathrm{D}$ & LES & $\begin{array}{l}\text { Martin et al. } \\
\text { [141] }\end{array}$ \\
\hline
\end{tabular}

Combustion Control Studies

\begin{tabular}{|l|l|l|}
\hline \multicolumn{1}{|c|}{ Control type } & \multicolumn{1}{c|}{ Method } & \multicolumn{1}{c|}{ Authors } \\
\hline Passive control & Fuel splitting & Sewell and Sobieski [7] \\
\hline Passive control & Fuel splitting & Cohen et al. [3] \\
\hline
\end{tabular}




\begin{tabular}{|l|l|l|}
\hline Passive control & Fuel splitting & Eisenhower et al. [142] \\
\hline Passive control & Fuel splitting & Noiray et al. [121] \\
\hline Passive control & Perforated plates & Putnam [143] \\
\hline Passive control & Quarter-wave tubes & Richards et al. [144] \\
\hline Passive control & Quarter-wave tubes & Mongia and coworkers [2, 78, 145] \\
\hline Passive control & Helmholtz resonators & Stow and Dowling [146] \\
\hline Passive control & Helmholtz resonators & Flohr and coworkers [5, 147] \\
\hline Passive control & Baffles & Dawson and Worth [103] \\
\hline Passive control & Pilot fuel & Pandalai and Mongia [145] \\
\hline Passive control & Pilot fuel & Smith and Blust [4] \\
\hline Passive control & Impedance tuning & Bothien et al. [121] \\
\hline Passive control & Asymmetric nozzle & Hermann et al. [148] \\
\hline Active control & Acoustic forcing & Gelbert et al. [149] \\
\hline Active control & Acoustic forcing & Illingworth and Morgans [150] \\
\hline Active control & Fuel forcing & $\begin{array}{l}\text { Hermann and coworkers [148, 151- } \\
\text { 153] }\end{array}$ \\
\hline Active control & Fuel forcing & Morgans and Stow [154] \\
\hline
\end{tabular}


Table 2 - Injector coupling scenarios for acoustically compact, small area-ratio nozzles

\begin{tabular}{|l|l|l|l|}
\hline \multicolumn{1}{|c|}{ Impedance } & \multicolumn{1}{c|}{$\hat{Z}_{t r} / \rho_{0} c_{0} \square 1$} & $\hat{Z}_{t r} / \rho_{0} c_{0} \square 1$ & $\hat{Z}_{t r} / \rho_{0} c_{0}=1$ \\
\hline $\begin{array}{l}\text { Pressure } \\
\text { Pressure anti- }\end{array}$ & $\begin{array}{l}\text { Pressure-Coupled; } \\
\text { not quasi 1-D }\end{array}$ & $\begin{array}{l}\text { Pressure-Coupled; } \\
\text { quasi 1-D }\end{array}$ & $\begin{array}{l}\text { Pressure-Coupled; } \\
\text { quasi 1-D }\end{array}$ \\
\hline Traveling & $\begin{array}{l}\text { Pressure-Coupled, } \\
\text { not quasi 1-D }\end{array}$ & $\begin{array}{l}\text { Pressure-Coupled, } \\
\text { quasi 1-D }\end{array}$ & $\begin{array}{l}\text { Pressure-Coupled; } \\
\text { quasi 1-D }\end{array}$ \\
\hline Pressure node & \multicolumn{2}{|l|}{ Not pressure coupled, results independent of nozzle impedance } \\
\hline
\end{tabular}


Table 3 - Modal amplitude, $\hat{C}_{m_{h}}$, for decomposition of plane waves into strength of helical modes.

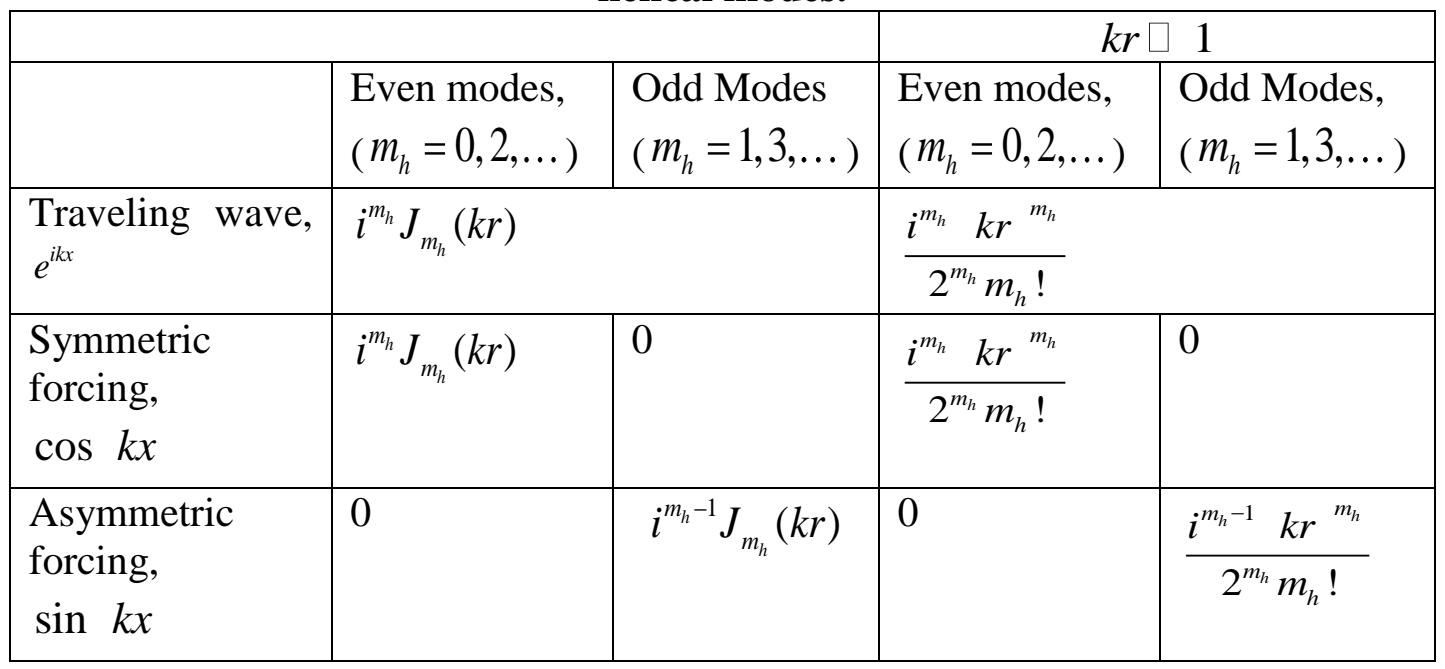

